\title{
Pointwise normality and Fourier decay for self-conformal measures
}

\author{
Amir Algom, Federico Rodriguez Hertz, and Zhiren Wang
}

\begin{abstract}
Let $\Phi$ be a $C^{1+\gamma}$ smooth IFS on $\mathbb{R}$, where $\gamma>0$. We provide mild conditions on the derivative cocycle that ensure that every self conformal measure is supported on points $x$ that are absolutely normal. That is, for every integer $p \geq 2$ the sequence $\left\{p^{k} x\right\}_{k \in \mathbb{N}}$ equidistributes modulo 1. We thus extend several state of the art results of Hochman and Shmerkin 29] about the prevalence of normal numbers in fractals. When $\Phi$ is self-similar we show that the set of absolutely normal numbers has full Hausdorff dimension in its attractor, unless $\Phi$ has an explicit structure that is associated with some integer $n \geq 2$. These conditions on the derivative cocycle are also shown to imply that every self conformal measure is a Rajchman measure, that is, its Fourier transform decays to 0 at infinity. When $\Phi$ is self similar and satisfies a certain Diophantine condition, we establish a logarithmic rate of decay.
\end{abstract}

\section{Introduction}

\subsection{Background}

Let $p$ be an integer greater or equal to 2 . Let $T_{p}$ be the times- $p$ map,

$$
T_{p}(x)=p \cdot x \quad \bmod 1, x \in \mathbb{R} .
$$

A number $x \in \mathbb{R}$ is called $p$-normal, or normal to base $p$, if its orbit $\left\{T_{p}^{k}(x)\right\}_{k \in \mathbb{N}}$ equidistributes for the Lebesgue measure on $[0,1]$. We call $x$ absolutely normal if it is $p$-normal for all integers $p \geq 2$. In 1909 Borel proved that Lebesgue almost every $x$ is absolutely normal. It is believed that this phenomenon should continue to hold true for typical elements of well structured sets with respect to appropriate measures, in the absence of obvious obstructions. Thus, we will call a Borel probability measure $\nu$ on $\mathbb{R}$ pointwise absolutely normal if $\nu$ almost every $x$ is absolutely normal. One of the main purposes of this paper is to specify a large and natural class of fractal measures that are pointwise absolutely normal. Additionally, we will indicate a large family of fractal sets that are typically Lebesgue null, such that set of absolutely normal numbers intersects them with full Hausdorff dimension.

There are two known general techniques to study whether a given Borel probability measure $\mu$ on $\mathbb{R}$ is supported on numbers normal to a given base $p$ : The first method involves establishing sufficiently fast decay of the $L^{2}(\mu)$ norms of certain trigonometric polynomials as in Weyl's criterion. This method was famously used by Cassels and Schmidt [55, 14] independently to show that if $\mu$ is the Cantor-Lebesgue measure on the middle- $\frac{1}{3}$ Cantor set, then $\mu$ almost every $x$ is $p$ -

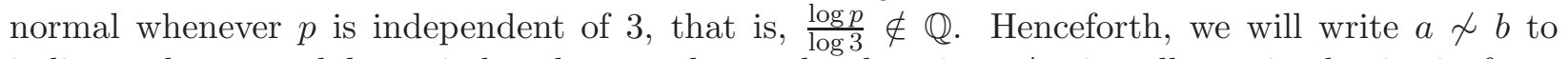
indicate that $a$ and $b$ are indepedent, and $a \sim b$ otherwise. A virtually optimal criteria for a measure to be supported on numbers that are $p$-normal in terms of these $L^{2}$ norms was later formulated by Davenport-Erdős-LeVeque [18], and was used by several authors including Brown, Pearce, Pollington, and Moran [47, 10, 11]. An excellent exposition to this subject is given in Bugeaud's book [13. 
The Davenport-Erdös-LeVeque criteria is closely related to the decay rate of the Fourier transform of Borel probability measures on $\mathbb{R}$ : Let $\nu$ be such a measure. For every $q \in \mathbb{R}$ the Fourier transform of $\nu$ at $q$ is defined by

$$
\mathcal{F}_{q}(\nu):=\int \exp (2 \pi i q x) d \nu(x)
$$

The measure $\nu$ is called a Rajchman measure if $\lim _{|q| \rightarrow \infty} \mathcal{F}_{q}(\nu)=0$. By the Riemann-Lebesgue Lemma, if $\nu$ is absolutely continuous then it is Rajchman. On the other hand, by Wiener's Lemma if $\nu$ has an atom then it is not Rajchman. For measures that are both continuous (no atoms) and singular, determining whether or not $\nu$ is a Rajchman measure may be a challenging problem. The Rajchman property and, when available, further information about the rate of decay of $\mathcal{F}_{q}(\nu)$, have various consequences on the geometry of $\nu$ [1]. Returning to the Davenport-Erdös-LeVeque Theorem, it ensures that if e.g. there is some $\alpha=\alpha(\nu)>0$ such that

$$
\left|\mathcal{F}_{q}(\nu)\right| \leq O\left(\frac{1}{|\log \log | q||^{1+\alpha}}\right), \text { as }|q| \rightarrow \infty
$$

then $\nu$ is pointwise absolutely normal. We note, however, that such bounds are usually hard to obtain (if they are true at all) in concrete situations, even for naturally defined measures. The third objective of this paper, which arises in conjuncture with the number theoretic framework discussed above, is to establish the Rajchman property for a class of dynamically defined measures.

In 2015 Hochman and Shmerkin [29] introduced a new method, giving a fractal geometric condition that is sufficient for a measure to be supported on $p$-normal numbers. This condition applies to a wide class of measures that arise from some dynamical or arithmetic origin. One of the virtues of this method is that it can be used regardless of knowledge on the behaviour of the Fourier transform of the underlying measure. Instead, one needs to understand the so-called scenery of the measure at typical points [29, Section 1.2]. In order to compute the scenery in specific examples, one usually works with measures such that their small "pieces" have mild (or no) overlaps, and this computation can become difficult in the presence of complicated overlaps. Many of the results of Hochman-Shmerkin still remain the state of the art on the subject, and we will recall them as we compare them to our results.

In this paper we introduce a new dynamical condition for a self-conformal measure (defined below) to be both a Rajchman measure and pointwise absolutely normal. A rate of decay is only established in some special cases, so in general we cannot invoke Davenport-Erdős-LeVeque to get absolute normality. Thus, we will prove pointwise absolute normality directly, regardless of the decay rate of the Fourier transform. This provides many new examples of both Rajchman measures and of pointwise absolutely normal measures, and allows us to extend result:1 of HochmanShmerkin, as detailed below. We then proceed to show that self similar sets intersect the set of absolutely normal numbers with full Hausdorff dimension, unless an obvious obstruction is present.

Self-conformal measures are defined as follows: Let $\Phi=\left\{f_{1}, \ldots, f_{n}\right\}$ be a finite set of strict contractions of a compact interval $I \subseteq \mathbb{R}$ (an IFS), such that every $f_{i}$ is differentiable. We say that $\Phi$ is $C^{\alpha}$ smooth if every $f_{i}$ is at least $C^{\alpha}$ smooth for some $\alpha \geq 1$. It is well known that there exists a unique compact set $\emptyset \neq K=K_{\Phi} \subseteq I$ such that

$$
K=\bigcup_{i=1}^{n} f_{i}(K) .
$$

\footnotetext{
${ }^{1}$ Hochman and Shmerkin can work with more general $\beta$ transformation, that is, maps of the form $x \mapsto \beta x \bmod 1$ for certain $\beta>1$. We will compare this to our method in Section 1.4.
} 
The set $K$ is called a self-conformal set, and the attractor of the IFS $\left\{f_{1}, \ldots, f_{n}\right\}$. In the special case where each $f_{i}$ is affine, i.e. $f_{i}(x)=r_{i} \cdot x+t_{i}$ and $0<\left|r_{i}\right|<1$, we call $K$ a self-similar set. We always assume that there exist $i \neq j$ such that the fixed point of $f_{i}$ is not equal to the fixed point of $f_{j}$. This ensures that $K$ is infinite. We call $\Phi$ uniformly contracting if

$$
0<\inf \left\{\left|f^{\prime}(x)\right|: f \in \Phi, x \in I\right\} \leq \sup \left\{\left|f^{\prime}(x)\right|: f \in \Phi, x \in I\right\}<1 .
$$

Finally, following Hochman-Shmerkin [29], we say that $\Phi$ is regular if it is uniformly contracting, and the intervals $f_{i}(I)$ are disjoint except possibly at their endpoints (so that, in particular, the so-called open set condition is satisfied).

Next, let $\mathbf{p}=\left(p_{1}, \ldots, p_{n}\right)$ be a strictly positive probability vector, that is, $p_{i}>0$ for all $i$ and $\sum_{i} p_{i}=1$. It is well known that there exists a unique Borel probability measure $\nu$ such that

$$
\nu=\sum_{i=1}^{n} p_{i} \cdot f_{i} \nu, \quad \text { where } f_{i} \nu \text { is the push-forward of } \nu \text { via } f_{i} .
$$

The measure $\nu$ is called a self-conformal measure, and is supported on $K$. If every $f_{i}$ is affine then $\nu$ is called a self-similar measure. Since $K$ is always assumed to infinite, the assumption that $p_{i}>0$ for every $i$ implies that $\nu$ is non-atomic. In particular, all self-conformal measures in this paper are non-atomic.

\subsection{Pointwise normality and Fourier decay for self conformal measures}

\subsubsection{Main technical Theorem}

We first formulate a general condition that ensures a given self conformal measure is both Rajchman and pointwise absolutely normal. Let $\Phi=\left\{f_{1}, \ldots, f_{n}\right\}$ be an IFS on an interval $I$ such that each $f_{i}$ is differentiable. For every $\omega \in\{1, \ldots, n\}^{\mathbb{N}}$ and $m \in \mathbb{N}$ let

$$
f_{\left.\omega\right|_{m}}=f_{\omega_{1}} \circ \circ \circ f_{\omega_{m}} .
$$

Fix $x_{0} \in I$. Then we have a surjective coding map $\{1, \ldots, n\}^{\mathbb{N}} \rightarrow K$ defined by

$$
\omega \in\{1, \ldots, n\}^{\mathbb{N}} \mapsto x_{\omega}:=\lim _{m \rightarrow \infty} f_{\left.\omega\right|_{m}}\left(x_{0}\right) .
$$

Assuming the IFS is uniformly contracting and $C^{1+\gamma}$ smooth, let $\rho:=\left(\sup _{f \in \Phi}\left\|f^{\prime}\right\|_{\infty}\right)^{\gamma} \in(0,1)$, and define a metric on $\{1, \ldots, n\}^{\mathbb{N}}$ via

$$
d_{\rho}\left(\omega, \omega^{\prime}\right):=\rho^{\min \left\{n: \omega_{n} \neq \omega_{n}^{\prime}\right\}} .
$$

Let $\mathbf{p}$ be a strictly positive probability vector on $\{1, \ldots, n\}$, and let $\nu$ be the corresponding self conformal measure. Let $\mathbb{P}=\mathbf{p}^{\mathbb{N}}$ be the product measure on $\{1, \ldots, n\}^{\mathbb{N}}$. Then $\nu$ is the push-forward of $\mathbb{P}$ via $\omega \mapsto x_{\omega}$. Also, for every $1 \leq a \leq n$ let $\iota_{a}:\{1, \ldots, n\}^{\mathbb{N}} \rightarrow\{1, \ldots, n\}^{\mathbb{N}}$ be the map

$$
\iota_{a}\left(\omega_{1}, \omega_{2}, \cdots\right)=\left(a, \omega_{1}, \omega_{2}, \cdots\right) .
$$

Let $G$ to be the free semigroup generated by the family $\left\{\iota_{a}: 1 \leq a \leq n\right\}$. We define the derivative cocycle $c: G \times\{1, \ldots, n\}^{\mathbb{N}} \rightarrow \mathbb{R}$ via

$$
c(a, \omega)=-\log \left|f_{a}^{\prime}\left(x_{\omega}\right)\right| .
$$


Choose some $\kappa \in(0,1]$ and let $H^{\kappa}$ denote the space of $\kappa$-Hölder continuous maps $\{1, \ldots, n\}^{\mathbb{N}} \rightarrow \mathbb{C}$, and define $\Lambda_{c} \subseteq \mathbb{R}$ via

$$
\begin{aligned}
\Lambda_{c}= & \left\{\theta: \text { There exists } \phi_{\theta} \in H^{\kappa} \text { with }\left|\phi_{\theta}\right|=1 \text { and } u_{\theta} \in S^{1}\right. \text { such that } \\
& \left.\phi_{\theta}\left(\iota_{a}(\omega)\right)=u_{\theta} \cdot e^{-i \theta \cdot c(a, \omega)} \cdot \phi_{\theta}(\omega), \quad \text { for all }(a, \omega) \in\{1, \ldots, n\} \times\{1, \ldots, n\}^{\mathbb{N}}\right\} .
\end{aligned}
$$

It is clear that $0 \in \Lambda_{c}$. If $\Lambda_{c}=\{0\}$ then the cocycle $c$ is aperiodic in the sense of Benoist-Quint [3, Equation (15.8)], and this will be used in an essential way to prove the following Theorem:

Theorem 1.1. Let $\Phi$ be a uniformly contracting $C^{1+\gamma}$ smooth IFS for some $\gamma>0$, and let $\nu$ be a self conformal measure. If $\Lambda_{c}=\{0\}$ then:

1. $\nu$ is a Rajchman measure, that is, $\lim _{|q| \rightarrow \infty} \mathcal{F}_{q}(\nu)=0$.

2. $\nu$ is pointwise absolutely normal.

Note that the conditions of Theorem 1.1 are invariant under conjugation by $C^{1+\gamma}$ maps with non-vanishing derivative, a useful feature in applications. Before turning to these applications, we say a few words about what goes into the proof: For part (1), the most important ingredient is Theorem 3.7, a conditional local limit Theorem for certain random variables resembeling stopping times that are related to a random walk driven by the derivative cocycle. That is, this local equidistrubtion property holds up to conditioning on "good" cells of suitable partitions of the space $\{1, \ldots, n\}^{\mathbb{N}}$. These good cells are produced via a central limit theorem for cocycles, and the local equidistribution follows from a local limit theorem for aperiodic cocycles, both proved by Benoist-Quint [3, Theorem 12.1 and Theorem 16.15]. This is the only part of the proof where the assumption $\Lambda_{c}=\{0\}$ is used. The rest of the proof consists of subtle linerization arguments and an adaptation of a Lemma of Hochman [27, regarding oscillatory integrals that arise at the end of the proof. See Section 4 for more details.

For part (2), fixing a $\nu$ typical point $x$ and an integer base $p$, we first employ a martingale argument in the spirit of Hochman-Shmerkin [29, Theorem 2.1] and a further linearization step. This reduces the problem to that of proving the Rajchman property with the same rate for a countable family of measure that arise as $T_{p}^{n}$-magnifications of increasingly small pieces of the measure about the point $x$. The treatment of the Fourier transform of these measures relies on a similar scheme as in part (1), but the additional steps further complicate the already delicate analysis involved. This necessitates the introduction of several new ideas. The most important one is Theorem 3.8, another conditional local limit Theorem that is tailored to this situation. See Section 5 for more details.

\subsubsection{Applications and related results}

We proceed to describe some applications of Theorem 1.1. Before doing so, we introduce two new definitions: Let $\Phi$ be a self similar IFS with corresponding contraction ratios $\left\{r_{1}, \ldots, r_{n}\right\}$. We say that $\Phi$ is periodic if there exists some $r \in \mathbb{R}$ such that

$$
\left\{\log \left|r_{1}\right|, \ldots, \log \left|r_{n}\right|\right\} \subset r \mathbb{Z}
$$

otherwise, we say that $\Phi$ is aperiodic. Note that $\Phi$ is aperiodic if and only if there are $i \neq j$ such that $\frac{\log \left|r_{i}\right|}{\log \left|r_{j}\right|} \notin \mathbb{Q}$. We call $\Phi$ Diophantine if there are $l, C>0$ such that

$$
\inf _{y \in \mathbb{R}} \max _{i \in\{1, \ldots n\}} d\left(\log \left|r_{i}\right| \cdot x+y, \mathbb{Z}\right) \geq \frac{C}{|x|^{l}}, \text { for all } x \in \mathbb{R} \text { large enough in absolute value. }
$$


Notice that if $\Phi$ is Diophantine then it is aperiodic, but the converse is false in general. This Diophantine condition is adopted from Breuillard's work [7, Section 3.1]. It is generic in the sense that it holds if $n \geq 3$ and we draw $\left\{\log \left|r_{1}\right|, \ldots, \log \left|r_{n}\right|\right\}$ according to the Lebesgue measure on $\mathbb{R}^{n}$, Proposition 2.4], and is met if e.g. $\log \left|r_{1}\right|, \ldots, \log \left|r_{n}\right|$ are rationally independent algebraic numbers [1, Proposition 2.7]. See also Section 6.3 for a family of examples related to the work of Moser [42]. We are now ready to summarize some corollaries of Theorem 1.1 .

Corollary 1.2. Let $\Phi$ be a uniformly contracting $C^{1+\gamma}$ smooth IFS for some $\gamma>0$, and let $\nu$ be a self conformal measure.

1. Suppose that for every $t, r \in \mathbb{R}$, the set

$$
\left\{\log \left|f^{\prime}(y)\right|: \text { where } f(y)=y, \quad f \in \Phi\right\}
$$

is not included in the set $t+r \mathbb{Z}$. Then $\nu$ is both pointwise absolutely normal and Rajchman.

2. Suppose that $\Phi$ is an aperiodic self similar IFS (so $\nu$ is a self similar measure), and let $g: I \rightarrow \mathbb{R}$ be a $C^{1+\gamma}$ smooth map, where $\gamma>0$. If $g^{\prime}$ does not vanish then $g \nu$ is both pointwise absolutely normal and Rajchman.

If $\Phi$ is Diophantine then there exists some $\alpha=\alpha(\nu)>0$ such that

$$
\left|\mathcal{F}_{q}(\nu)\right| \leq O\left(\frac{1}{|\log | q||^{\alpha}}\right), \text { as }|q| \rightarrow \infty
$$

3. Suppose that $\Phi$ is $C^{\omega}$ smooth, or that it is $C^{2}$ and $K_{\Phi}$ is an interval. If $\nu$ is not pointwise absolutely normal or not Rajchman then $\Phi$ is $C^{\omega}$ or $C^{2}$ (depending on the smoothness of $\Phi$ ) conjugate to a periodic self similar IFS.

We emphasize that no separation condition is imposed on $\Phi$. The deduction of Corollary 1.2 from Theorem 1.1 relies on the study of the derivative cocycle under these assumptions, and this analysis is carried out in Section 6.

Part (1) of Corollary [1.2 should be compared with a result [29, Theorem 1.4] of HochmanShmerkin: For a given $p$, if

$\Phi$ is regular and some element of $\left\{f^{\prime}(y)\right.$ : where $\left.f(y)=y, \quad f \in \Phi\right\}$ is independent of $p$

then $\nu$ almost every point is $p$-normal for every self conformal measure $\nu$. Notice that the assumption in part (1) implies this arithmetic condition holds for every $p$. So, in its setting, part (1) extends the result of Hochman-Shmerkin by removing the separation assumption from the IFS. Furthermore, [29, Theorem 1.4] remains true when pushing the measure $\nu$ forward via any real diffeomorphism $g$. Corollary 1.2 part (1), in contrast, remains true when pushing the measure forward via a $C^{1+\gamma}$ diffeomorphism, but we don't know if this is true for $C^{1}$ diffeomorphisms. Finally, we remark that [29, Theorem 1.4] holds for a more general class of transformations and measures, and we will discuss this in Section [1.4.

The normality assertion of part (2) should be compared with another result [29, Theorem 1.7] of Hochman-Shmerkin, where they prove that for every self similar measure with respect to a regular self similar IFS, its push-forward under a non-affine $C^{\omega}$ map is pointwise absolutely normal. So, [29, Theorem 1.7] does not require the IFS to be aperiodic, but does require a more restrictive regularity (separation) assumption. In addition, the smoothness assumption on the perturbing 
map in part (2) is less restrictive than [29, Theorem 1.7]. For example, let $\nu$ be any self conformal measure with respect to the aperiodic self similar IFS

$$
\left\{\frac{x}{2}, \frac{x+1}{3}, \frac{x+1}{5}\right\} .
$$

Corollary 1.2 part (2) implies that $f(x)$ is absolutely normal for $\nu$ almost every $x$ and any diffeomorphism $f \in C^{1+\gamma}(\mathbb{R})$. Notice that since the IFS is not regular and the map $f$ might not be $C^{\omega}[29$, Theorem 1.7] does not apply in this situation. On the other hand, consider the Cantor-Lebesgue measure $\mu$ on the middle $-\frac{1}{3}$ Cantor set. By [29, Theorem 1.7] $x^{2}$ is absolutely normal for $\mu$ almost every $x$. Notice that since the underlying regular IFS

$$
\left\{\frac{x}{3}, \frac{x+2}{3}\right\}
$$

is not aperiodic, Corollary 1.2 part (2) does not apply here.

The Rajchman assertion of part (2) should be compared with a recent Theorem of Li-Sahlsten [40, Theorem 1.2] (see also [6]): They proved that for an orientation preseving aperiodic self similar IFS any self-similar measure is a Rajchman measure. So, Part (2) extends the Li-Sahlsten Theorem to all $C^{1+\gamma}$ smooth images. Corollary 1.2 part (2) also complements a classical Theorem of Kaufman [34 that was later extended by Mosquera-Shmerkin [43] (see also [15]) about polynomial Fourier decay for $C^{2}$ images of homogeneous (i.e. $r_{i}=r_{j}$ for all $i, j$ ) self similar measures. We thus partially answer a folklore open question (see e.g. [52]) about the existence of a Kaufman Theorem in the non homogeneous setting.

The quantitative assertion of part (2) should be compared with another Theorem of Li-Sahlsten [40, Theorem 1.3] where a similar logarithmic decay rate was obtained, but under a different Diophantine assumption. In Section 6.3 we will give a family of Diophantine IFS's that do not satisfy the conditions of [40, Theorem 1.3] since $\frac{\log \left|r_{i}\right|}{\log \left|r_{j}\right|}$ is either rational or a Liouville number for all $i, j$. Thus, via Corollary 1.2 part (2) we obtain many new examples of self similar measures with logarithmic Fourier decay. Finally, we will discuss the problem of getting an effective decay rate for non-linear IFS's in Section 1.4.

The Rajchman question for self similar measures is a classical problem that has received much attention over the years: Consider, for example, the family of Bernoulli convolutions $\left\{\nu_{r}\right\}_{r \in(0,1)}$ : For every $0<r<1$ we define the self similar IFS $\{r \cdot x-1, r \cdot x+1\}$ with the probability vector $\mathbf{p}=\left(\frac{1}{2}, \frac{1}{2}\right)$. It is a fundemental problem to determine for which $r \in\left(\frac{1}{2}, 1\right)$ is $\nu_{r}$ is absolutely continuous. A celebrated result of Erdös [21] says that if $r^{-1}$ is a Pisot number then $\nu_{r}$ is not a Rajchman measure and consequently is not absolutely continuous. Recall that a Pisot number is a real algebraic integer greater than one whose Galois conjugates all lie inside the unit disc. Later, Salem [53] completed the picture in terms of the Rajchman property, by showing that $\nu_{r}$ is not Rajchman only if $r^{-1}$ is Pisot (see also the related works of Piatetski-Shapiro 67] and Salem and Zygmund [54). We remark that through some ground breaking recent papers (e.g. [26], [58, 8], 65. to name a few) the geometric properties of $\nu_{r}$ are now far better understood. However, the question of absolute continuity remains open. More general self similar measures were studied by Strichartz [61, 62]: He proved that their Fourier transforms decay on average, with a recent large deviations estimate on this decay given by Tsujii 64] (see also 63. for a related paper about self conformal measures). However, these papers do not establish the Rajchman property, since they exclude certain frequencies.

Very recently, as we have already mentioned, Li-Sahlsten [40] proved the Rajchman property in the presence of indepedent contractions. In the complementary case, when all contractions are powers of some $r \in(0,1)$, Brémont [6] proved that a self-similar measure can fail to be Rajchman 
only if $r^{-1}$ is Pisot. In fact, Brémont fully characterised the IFS up to affine conjugation, and this will play a crucial role later in this paper. Another proof of this fact was given by Varjú-Yu [66]. Finally, we note that Li and Sahlsten [39] also generalized their results to self affine measures in higher dimensions.

The problem of quantitative Fourier decay for self similar measures is also a classical one: For Bernoulli convolutions, it follows from the works of Erdös [22] and Kahane [32] that $\nu_{r}$ has polynomial decay outside a set of zero Hausdorff dimension (see also [16], [12] [17] for rates in some explicit examples of $r$ ). In the complementary case to the afformentioned effective result of Li-Sahlsten [40, Theorem 1.3], when all contractions are powers of some $r \in(0,1)$, Varjú-Yu [66] proved logarithmic decay as long as $r^{-1}$ is not a Pisot or a Salem number. Finally, Solomyak 60] has recently established polynomial decay for all self-similar measures except for a zero Hausdorff dimensional exceptional set of contraction ratios.

Next, the normality assertion of Corollary 1.2 part (3) is related to another Theorem of Hochman-Shmerkin [29, Theorem 1.6], where a similar result is proved for regular $C^{\omega}$ IFS's that contain non-affine maps. Apart from removing the separation assumption, the $C^{2}$ case and the classification of the conjugated IFS as in part (3) seem to be completely new.

The Rajchman assertion of part (3) gives many new examples of self conformal Rajchman measures. It also provides a unified proof to several pre-existing results regarding the Rajchman property for $C^{\omega}$ IFS's that are not conjugate to a self similar IFS. These include those of SahlstenStevens [52] (for a class of regular $C^{\omega}$ self-conformal measures that are not conjugate to linear), and in some cases those of $\mathrm{Li}$ [37, 36] (Furstenberg measures for $S L(2, \mathbb{R})$ cocycles under mild assumption - see [69, 2] for conditions ensuring that such measures satsify the conditions of part (3)). Part (3) is also closley related to the work of Bourgain-Dyatlov [5] (who study PattersonSullivan measures for convex cocompact Fuchsian groups, see also [38]). However, we do not recover the polynomial decay rate proved in [5, 37, 52. Finally, generlizing the work of Kaufman [33] and later Queffélec-Ramaré [48, Jordan-Sahlsten [31] and later Sahlsten-Stevens [51] proved polynomial decay for certain Gibbs measures for the Gauss map $x \mapsto \frac{1}{x} \bmod 1$ on the interval. These can be considered as self-conformal measures with respect to an IFS with countably many maps, see also [29, Theorem 1.12].

\subsection{Dimension of absolutely normal numbers inside self similar sets}

Let us now specialize to self similar sets. Let $\lambda$ denote the Lebesgue measure on $\mathbb{R}$, and recall that Borel's normal number Theorem asserts that $\lambda$-a.e. $x$ is absolutely normal. However, Borel's Theorem gives no information about absolutely normal numbers inside sets that are Lebesgue null. The following rigidity result says that absolutely normal numbers have full Hausdorff dimension inside a given self similar set, unless the underlying IFS has a very specific structure. We use the standard notation $\operatorname{dim} X$ for the Hausdorff dimension of a set $X$, and

$$
\operatorname{dim} \mu=\inf \{\operatorname{dim} X: \mu(X)>0\}
$$

for the (lower) Hausdorff dimension of a Borel probability measure $\mu$. Now, given an orientation preserving self similar IFS $\Phi$ let $\Phi_{1}:=\Phi$, and for every integer $m \geq 2$,

$$
\Phi_{m}:=\left\{g: g=\phi_{1} \circ \ldots \circ \phi_{\ell}, \text { such that } \phi_{i} \in \Phi, g^{\prime}(0)<\frac{1}{m} \text { and }\left(\phi_{1} \circ \ldots \circ \phi_{\ell-1}\right)^{\prime}(0) \geq \frac{1}{m}\right\} .
$$

Observe that for every $m \in \mathbb{N}, K_{\Phi_{m}}=K_{\Phi}$, i.e. all these IFS's have the same attractor as $\Phi$. 
Theorem 1.3. Let $\Phi$ be an orientation preserving self similar IFS with attractor $K$. If

$$
\operatorname{dim} K \cap\{x: x \text { is absolutely normal }\}<\operatorname{dim} K
$$

then there exists some $m \in \mathbb{N}$ such that the IFS $\Phi_{m}=\left\{g_{i}(x)=r_{i} x+t_{i}\right\}$ has the following structure:

(i) There is an integer $n \geq 2$ such that every $r_{i}=n^{-k_{i}}$ and the $k_{i} \in \mathbb{N}$ are relatively prime.

(ii) Every $t_{i}$ is not $n$-normal. If there exist $r_{k} \neq r_{j}$ and $\Phi_{m}$ is regular then every $t_{i} \in \mathbb{Q}$.

If $\Phi$ admits a self similar measure $\mu$ with $\operatorname{dim} \mu=\operatorname{dim} K$ then this holds for $m=1$. That is, the original IFS $\Phi$ already has this structure.

For example, if $\Phi$ is regular and admits two different contraction ratios, then (3) implies that all contraction ratios are powers of some integer $n \geq 2$ and all translations are rational. In general, if $\Phi$ does not have exact overlaps (i.e. the semi-group generated by its maps is free), and if the exact overlaps conjecture [59] holds true, then one can always find a self similar measure $\mu$ with $\operatorname{dim} \mu=\operatorname{dim} K$. Hochman [26, Theorem 1.1] verified this conjecture under very weak regularity conditions, that hold true if e.g. all parameters of $\Phi$ are algebraic [26, Theorem 1.5] (Rapaport [49] recently showed that it suffices to assume only the contraction ratios are algebraic). The conjecture was also fully resolved for Bernoulli convolutions by the combined efforts of Hochman [26], Breuillard-Varjú [8], and finally Varjú [65] (see also [50]). Thus, in all the cases listed above, if $\Phi$ does not satisfy (i) and (ii) as in Theorem 1.3 , then

$$
\operatorname{dim} K \cap\{x: x \text { is absolutely normal }\}=\operatorname{dim} K .
$$

It is interesting to compare this with a result [9] of Broderick et al. (that extends a Theorem of Schmidt [56]): The set of real numbers not normal to any integer base intersects any infinite self-similar in a set of full Hausdorff dimension in the fractal.

The proof of Theorem 1.3 relies on the following Theorem, which is a bi-product of the argument proving Theorem 1.1.

Theorem 1.4. Let $\mu$ be a self similar measure. If $\mu$ is a Rajchman measure then it is pointwise absolutely normal.

This is a significant improvement to the Davenport-Erdős-LeVeque criterion, since no decay rate is required. Once Theorem 1.4 is established, we proceed to prove Theorem 1.3 by first showing that, under its assumptions, there is some $m$ and a self similar measure on $\Phi_{m}$ that is not Rajchman. We then combine the results of Li-Sahlsten [40] and those of Brémont [6] to prove that $\Phi_{m}$ is affinely conjugated to an IFS in so-called Pisot form [6, Definition 2.2]. We then show that the underlying Pisot number is in fact an integer, and characterize the affine conjugating map by appealing to the recent results of Dayan-Ganguly-Weiss [19]. See Section 7 for more details.

\subsection{Some further remarks}

It is natural to ask for a condition similar to (2) that would yield a quantitative decay rate for the Fourier transform of self conformal measures with respect to non-linear IFS's. To this end we require effective versions of the central and local limit Theorem for cocycles from [3], that are used to prove the Rajchman property. Now, in the self-similar case the random walk driven by the derivative cocycle is a classical random walk on the line. Thus, here we can substitute the central limit Theorem [3, Theorem 12.1] for the Berry-Esseen inequality [23], and the local limit Theorem 
[3. Theorem 16.15] for Breuillard's effective local limit Theorem [7, Théorème 4.2], which is why we require the Diophantine condition. Since the arguments of Breuillard [7] and of Benoist-Quint [3. Chapter 16] are closely related, it might be possible to prove an effective local limit Theorem for the derivative cocycle under a suitable Diophantine condition. We plan to study this problem in the near future.

Also, the method of Hochman-Shmerkin [29] for pointwise normality works for a broader class of $\beta$ transformations of the form $T_{\beta}(x)=\beta x \bmod 1$, when $\beta>1$ is a Pisot number. In its current form, our method seems less suitable to treat $T_{\beta}$ for non-integer $\beta$. The main issue is that the identity $T_{p}^{n}=T_{p^{n}}$, which is used several times in our proof (e.g. in Claim 5.7) and is trivial for integers $p \geq 2$, is not true for $T_{\beta}$ when $\beta$ is not an integer. We remark that it might be possible to substantially refine our proof to get around this issue, and leave this to future research.

The method of Hochman-Shmerkin also works for a broader class of measures. This class of measures is what they refer to as quasi-product measures, which is a more general class than Gibbs measures for Hölder potentials. We expect that our results extend to a broader class of measures as well, but do not pursue this goal in the present paper.

Finally, the referee has suggested that by incorporating ideas from renewal theory into the proof of the conditional local limit Theorem 3.7, one might be able to relax the aperiodicity assumption made in Theorem 1.1 into a more arithmetic assumption. We plan to explore this possibility in a future project.

Organization After some preliminaries in Section 2, the local limit Theorems previously alluded to are formulated and proved in Section 3. The subsequent Sections 4 and 5 contain the proof of Theorem 1.1 parts (1) and (2), respectively. After that, in Section 6 we derive Corollary 1.2 from Theorem 1.1 and provide some examples of Diophantine IFS's. The final Section 7 contains the proof of Theorem 1.3, and related constructions.

Acknowledgements The inspiration for this work came from Hochman's recent proof [27] of Host's equidistribution Theorem [30]. We thank Mike Hochman for providing us with a preprint of his work, and for some illuminating discussions about it. We also thank Tuomas Sahlsten and Connor Stevens for interesting discussions regarding the Rajchman property. We are grateful to Péter Varjú and the anonymous referee for their helpful remarks, that in particular allowed us to weaken our previous definition of aperiodicity for self similar IFS's to its current form, and thus strengthen Corollary 1.2. Finally, we thank the referee for suggesting an alternative proof of Theorem 1.1 part (2) using the tools of this paper that is outlined in Remark 5.6, and for pointing out a certain possible simplification of our Diophantine condition, discussed in Remark 6.7.

\section{Preliminaries}

Throughout this section we work with an IFS as in Theorem 1.1, and follow the notation introduced in Section 1.2.1. We also use the notation $\mathcal{A}=\{1, \ldots, n\}$. By uniform contraction there exists $D, D^{\prime} \in \mathbb{R}$ such that

$$
0<D:=\min \left\{-\log \left|f^{\prime}(x)\right|: f \in \Phi, x \in I\right\}, \quad D^{\prime}:=\max \left\{-\log \left|f^{\prime}(x)\right|: f \in \Phi, x \in I\right\}<\infty .
$$

Equivalently, for every $f \in \Phi$ and $x \in I$,

$$
0<e^{-D^{\prime}} \leq\left|f^{\prime}(x)\right| \leq e^{-D}<1 .
$$

We also define, for $\mathbb{P}$ a.e. $\omega$ and any fixed $x_{0} \in I$,

$$
\chi:=\lim _{n} \frac{-\log \left|f_{\left.\omega\right|_{n}}^{\prime}\left(x_{0}\right)\right|}{n}>0
$$


the corresponding Lyapunov exponent.

\subsection{Basic geometry of self-conformal measures}

Here, we recall two useful and well known results. The first is the bounded distortion property, which holds in our situation since every $f_{i}$ is at least $C^{1+\gamma}$ smooth and strictly contracting. We refer e.g. to [46, The discussion about equation (1.3)] for more details.

Theorem 2.1. There exists some $L=L(\Phi)>1$ such that: for any $k \in \mathbb{N}$ and for any word $\eta \in \mathcal{A}^{k}$

$$
L^{-1} \leq \frac{\left|f_{\eta}^{\prime}(x)\right|}{\left|f_{\eta}^{\prime}(y)\right|} \leq L, \quad \text { for any } x, y \in I .
$$

The second is another standard result, about the non-atomicity of self-conformal measures. It follows from e.g. [25, Proposition 2.2]:

Lemma 2.2. Let $\nu$ be a self conformal measure as in Theorem 1.1. Then $\nu$ is not atomic. That is, for every $\epsilon>0$ there is a $\delta>0$ such that for any $y \in I$,

$$
\nu\left(B_{\delta}(y)\right)<\epsilon
$$

where $B_{\delta}(y)$ is the open ball about $y$ of radius $\delta>0$.

Notice that here we using our standing assumptions that $K_{\Phi}$ is infinite and that $\mathbf{p}$ is a strictly positive probability vector on $\mathcal{A}=\{1, \ldots, n\}$.

\subsection{Linearization lemmas}

Recall that $\Phi$ is a family of differentiable contractions $I \rightarrow I$ satisfying

$$
0<e^{-D^{\prime}} \leq\left\|f^{\prime}\right\|_{C^{0}} \leq e^{-D}<1, \quad\|f\|_{C^{1+\gamma}} \leq C, \quad \forall f \in \Phi
$$

for some $D, D^{\prime}>0, \gamma \in(0,1)$ and $C>0$. Define

$$
\Phi^{* n}:=\left\{\phi_{1} \circ \cdots \circ \phi_{n}: \quad \phi_{1}, \cdots, \phi_{n} \in \Phi\right\} .
$$

We shall require the following $C^{0}$ linearization lemma:

Lemma 2.3. For every $\beta \in(0, \gamma)$ there exists $\epsilon \in(0,1)$ such that for all $n \geq 1, g \in \Phi^{* n}$ and $x, y \in I$ satisfying $|x-y|<\epsilon$,

$$
\left|g(x)-g(y)-g^{\prime}(y)(x-y)\right| \leq\left|g^{\prime}(y)\right| \cdot|x-y|^{1+\beta} .
$$

What Lemma 2.3 means is that for every $y \in B_{\epsilon}(x)$ the function $g$ may be approximated exponentially fast on $B_{\epsilon}(x)$ by an affine map with similarity ratio $g^{\prime}(y)$.

Proof. For the purpose of this proof only, it will be convenient to use the notation

$$
0<\kappa^{\prime}:=e^{-D^{\prime}} \leq\left\|\phi^{\prime}\right\|_{C^{0}} \leq \kappa:=e^{D}<1, \quad \forall \phi \in \Phi .
$$

Now, for all $x, y \in I$ and $\phi \in \Phi$, there is an intermediate value $z$ between $x$ and $y$ such that

$$
\begin{aligned}
\left|\phi(x)-\phi(y)-\phi^{\prime}(y)(x-y)\right| & =\left|\phi^{\prime}(z)(x-y)-\phi^{\prime}(y)(x-y)\right| \\
& \leq\left|\phi^{\prime}(z)-\phi^{\prime}(y)\right||x-y| \leq C|z-y|^{\gamma}|x-y| \\
& \leq C|x-y|^{1+\gamma} .
\end{aligned}
$$


Define a sequence $\beta_{0}>\beta_{1}>\beta_{2}>\cdots$ by $\beta_{0}=\gamma, \beta_{n}=\beta_{n-1}-b \kappa^{(n-1) \gamma}$, where the constant $b:=\left(1-\kappa^{\gamma}\right)(\gamma-\beta)>0$ is chosen such that $\lim \beta_{n}=\beta$. Choose $\epsilon \in\left(0, \frac{1}{e}\right)$ sufficiently small, such that

$$
\frac{4 C}{\kappa^{\prime}} \epsilon^{\gamma-\beta_{1}}<\min \left(1-\frac{1}{e}, \frac{\beta}{2}\right) .
$$

We will prove inductively that:

If $n \geq 0,|x-y|<\epsilon$ and $g \in \Phi^{n}$, then

$$
\left|g(x)-g(y)-g^{\prime}(y)(x-y)\right| \leq\left|g^{\prime}(y)\right| \cdot|x-y|^{1+\beta_{n}} .
$$

For the $n=0$ case, assume $|x-y|<\epsilon$ and $g \in \Phi^{* 0}=\{\operatorname{Id}\}$, then $\left|g(x)-g(y)-g^{\prime}(y)(x-y)\right|=$ $|(x-y)-(x-y)|=0$ and (7) holds.

Assume $n \geq 1$ and the lemma holds for $n-1$. Suppose $|x-y|=\delta<\epsilon$ and $g \in \Phi^{* n}$. Then $g=\phi \circ \tilde{g}$ where $\phi \in \Phi$ and $\tilde{g} \in \Phi^{*(n-1)}$, and

$$
\left|\tilde{g}(x)-\tilde{g}(y)-\tilde{g}^{\prime}(y)(x-y)\right| \leq\left|\tilde{g}^{\prime}(y)\right| \delta^{1+\beta_{n-1}} .
$$

In particular, it follows that

$$
|\tilde{g}(x)-\tilde{g}(y)| \leq\left|\tilde{g}^{\prime}(y)\right| \delta\left(1+\delta^{\beta_{n-1}}\right) .
$$

Combining (5) and (9), we get

$$
\begin{aligned}
& \left|g(x)-g(y)-g^{\prime}(y)(x-y)\right| \\
= & \left|\phi(\tilde{g}(x))-\phi(\tilde{g}(y))-\phi^{\prime}(\tilde{g}(y)) \tilde{g}^{\prime}(y)(x-y)\right| \\
\leq & \left|\phi(\tilde{g}(x))-\phi(\tilde{g}(y))-\phi^{\prime}(\tilde{g}(y))(\tilde{g}(x)-\tilde{g}(y))\right| \\
& \quad+\left|\phi^{\prime}(\tilde{g}(y))\right| \cdot\left|\tilde{g}(x)-\tilde{g}(y)-\tilde{g}^{\prime}(y)(x-y)\right| \\
\leq & C|\tilde{g}(x)-\tilde{g}(y)|^{1+\gamma}+\left|\phi^{\prime}(\tilde{g}(y))\right| \cdot\left|\tilde{g}^{\prime}(y)\right| \delta^{1+\beta_{n-1}} \\
\leq & C\left(\left|\tilde{g}^{\prime}(y)\right| \delta\left(1+\delta^{\beta_{n-1}}\right)\right)^{1+\gamma}+\left|g^{\prime}(y)\right| \delta^{1+\beta_{n-1}} .
\end{aligned}
$$

Because $0<\delta<\epsilon<1$, we know (10) is bounded by:

$$
\begin{aligned}
& \left|g(x)-g(y)-\left(D_{y} g\right)(x-y)\right| \\
\leq & 4 C\left|\tilde{g}^{\prime}(y)\right| \cdot\left|\tilde{g}^{\prime}(y)\right|^{\gamma} \delta^{1+\gamma}+\left|g^{\prime}(y)\right| \delta^{1+\beta_{n-1}} \\
= & \left(4 C\left|\phi^{\prime}(\tilde{g}(y))\right|^{-1}\left|\tilde{g}^{\prime}(y)\right|^{\gamma} \delta^{\gamma-\beta_{n}}+\delta^{\beta_{n-1}-\beta_{n}}\right)\left|g^{\prime}(y)\right| \delta^{1+\beta_{n}} \\
\leq & \left(\frac{4 C}{\kappa^{\prime}} \kappa^{(n-1) \gamma} \delta^{\gamma-\beta_{n}}+\delta^{\beta_{n-1}-\beta_{n}}\right)\left|g^{\prime}(y)\right| \delta^{1+\beta_{n}} .
\end{aligned}
$$

To complete the induction, it suffices to prove

$$
\frac{4 C}{\kappa^{\prime}} \kappa^{(n-1) \gamma} \delta^{\gamma-\beta_{n}}+\delta^{\beta_{n-1}-\beta_{n}} \leq 1 .
$$

We distinguish between the cases $\log \frac{1}{\delta} \geq \frac{1}{\beta_{n-1}-\beta_{n}}$ and $\log \frac{1}{\delta}<\frac{1}{\beta_{n-1}-\beta_{n}}$.

If $\log \frac{1}{\delta} \geq \frac{1}{\beta_{n-1}-\beta_{n}}$, then $\delta^{\beta_{n-1}-\beta_{n}} \leq \frac{1}{e}$. Moreover, by (6),$\frac{4 C}{\kappa^{\prime}} \kappa^{(n-1) \gamma} \delta^{\gamma-\beta_{n}} \leq \frac{4 C}{\kappa^{\prime}} \epsilon^{\gamma-\beta_{1}}<1-\frac{1}{e}$. Thus (12) holds in this case. 
Assume now $\log \frac{1}{\delta}<\frac{1}{\beta_{n-1}-\beta_{n}}$. Since $e^{-t} \leq 1-\frac{1}{2} t$ on $[0,1]$, we know

$$
\begin{aligned}
\delta^{\beta_{n-1}-\beta_{n}} & =e^{-\left(\log \frac{1}{\delta}\right)\left(\beta_{n-1}-\beta_{n}\right)} \\
& \leq 1-\frac{1}{2}\left(\log \frac{1}{\delta}\right)\left(\beta_{n-1}-\beta_{n}\right) \leq 1-\frac{1}{2}\left(\beta_{n-1}-\beta_{n}\right)=1-\frac{1}{2} b \kappa^{(n-1) \gamma} \\
& \leq 1-\frac{4 C}{\kappa^{\prime}} \kappa^{(n-1) \gamma} \epsilon^{\gamma-\beta_{1}} \leq 1-\frac{4 C}{\kappa^{\prime}} \kappa^{(n-1) \gamma} \delta^{\gamma-\beta_{n}} .
\end{aligned}
$$

Here we used the facts that $0<\delta<\epsilon<\frac{1}{e}, \beta_{n} \leq \beta_{1}<\gamma$ and assumption (6). Hence (12) holds in this case as well.

We have established the inductive statement (7). As $\beta<\beta_{n}$ and $|x-y|<\epsilon<1$, the lemma then follows.

An important ingredient in the proof of the second local limit Theorem 3.8, discussed in Section 3. is the following (much easier) $C^{1}$ counterpart:

Lemma 2.4. For all $n \geq 1, g \in \Phi^{* n}$ and $x, y \in I$,

$$
|\log | g^{\prime}(x)|-\log | g^{\prime}(y)|| \lesssim_{\Phi}|x-y|^{\gamma} .
$$

Remark 2.5. By the notation $A \lesssim_{\Phi} B$ we mean that the number $A$ is smaller than $C \cdot B$, where the multiplicative constant $C=C(\Phi)$ depends only on $\Phi$. Similar notation is used throughout the paper.

Proof. Fix $n \in \mathbb{N}$, and let $g \in \Phi^{* n}$. Then there is some $\eta \in \mathcal{A}^{*}$ such that $g=f_{\eta}$. By our assumptions on the maps in $\Phi$, we have

$$
\begin{aligned}
|\log | g^{\prime}(x)|-\log | g^{\prime}(y)|| & \leq \sum_{m=1}^{n}|\log | f_{\eta_{m}}^{\prime}\left(f_{\eta_{m-1}}(x)\right)|-\log | f_{\eta_{m}}^{\prime}\left(f_{\eta_{\mid m-1}}(y)\right)|| \\
& \leq \sum_{m=1}^{n} \frac{1}{\min _{z \in I}\left|f_{\eta_{m}}^{\prime}(z)\right|}|| f_{\eta_{m}}^{\prime}\left(f_{\left.\eta\right|_{m-1}}(x)\right)|-| f_{\eta_{m}}^{\prime}\left(f_{\left.\eta\right|_{m-1}}(y)\right)|| \\
& \leq \sum_{m=1}^{n} e^{D^{\prime}}\left|f_{\eta_{m}}^{\prime}\left(f_{\eta_{m-1}}(x)\right)-f_{\eta_{m}}^{\prime}\left(f_{\eta_{m-1}}(y)\right)\right| \\
& \leq \sum_{m=1}^{n} C \cdot e^{D^{\prime}}\left|f_{\eta_{m-1}}(x)-f_{\left.\eta\right|_{m-1}}(y)\right|^{\gamma} \\
& \leq C \cdot e^{D^{\prime}} \sum_{m=1}^{n} e^{-D \cdot m \cdot \gamma}|x-y|^{\gamma} \leq C_{0}|x-y|^{\gamma}
\end{aligned}
$$

for some global constant $C_{0}$ that is independent of $n$, completing the proof.

\subsection{The Fourier transform of scaled measures}

The following Lemma is adapted from a recent paper of Hochman [27. For any $s, x \in \mathbb{R}$ let $M_{s}(x)=s \cdot x$ denote the multiplication map, and for any metric space $X$ let $\mathcal{P}(X)$ denote the space of Borel probability measures on $X$. 
Lemma 2.6. [27, Lemma 3.2] Let $\theta \in \mathcal{P}(\mathbb{R}), k>0$ and $\chi, D$ as in the previous Sections. Then for any $r>0$ and $q \neq 0$,

$$
\int_{k \cdot \chi}^{k \cdot \chi+D^{\prime}}\left|\mathcal{F}_{q}\left(M_{e^{-t}} \theta\right)\right|^{2} d t \leq D^{\prime} \cdot\left(\frac{e^{2}}{r \cdot|q|}+\int \theta\left(B_{e^{\chi^{k} \cdot r}}(y)\right) d \theta(y)\right) .
$$

In fact, Hochman's Lemma states that for any $\theta \in \mathcal{P}(\mathbb{R})$, any $r>0$, an any $m \neq 0$,

$$
\int_{0}^{1}\left|\mathcal{F}_{m}\left(M_{p^{t}} \theta\right)\right|^{2} d t \leq \frac{2}{r \cdot|m| \cdot \log p}+\int \theta\left(B_{r}(y)\right) d \theta(y)
$$

where here $p>1$.

In the context of Lemma 2.6, we apply this result for $p=e^{-1}$ and the measure $M_{e^{-k \chi}} \theta$ between the scales 0 and $D^{\prime}$. Then the same proof yields

$$
\begin{aligned}
\int_{k \cdot \chi}^{k \cdot \chi+D^{\prime}}\left|\mathcal{F}_{m}\left(M_{e^{-t}} \theta\right)\right|^{2} d t & =\int_{0}^{D^{\prime}}\left|\mathcal{F}_{m}\left(M_{e^{-t}}\left(M_{e^{-k \chi}} \theta\right)\right)\right|^{2} d t \\
& \leq D^{\prime} \cdot\left(\frac{e^{2}}{r \cdot|m|}+\int M_{e^{-k \chi}} \theta\left(B_{r}(y)\right) d M_{e^{-k \chi}} \theta(y)\right) \\
& =D^{\prime} \cdot\left(\frac{e^{2}}{r \cdot|m|}+\int \theta\left(B_{e^{\chi k} \cdot r}(y)\right) d \theta(y)\right)
\end{aligned}
$$

which is Lemma 2.6.

\section{Two local limit theorems}

We continue to work with an IFS $\Phi$ as in Theorem 1.1, and follow the notation introduced in Sections 1.2.1 and 2. The purpose of this Section is to establish two local limit Theorems: Theorem 3.7. which will be used to prove the Rajchman part of Theorem 1.1, and Theorem 3.8 which will be used to prove the normality part of Theorem 1.1. Our analysis relies on the central and local limit Theorems for cocycles with target proved by Benoist-Quint [3].

\subsection{Statements of the local limit Theorems}

We define the following functions and stopping times on $\mathcal{A}^{\mathbb{N}}$ :

$$
\begin{aligned}
& S_{n}(\omega)=-\log \left|f_{\left.\omega\right|_{n}}^{\prime}\left(x_{\sigma^{n}(\omega)}\right)\right| ; \quad \tau_{k}(\omega)=\min \left\{n: S_{n}(\omega) \geq k \chi\right\} ; \\
& \widetilde{S}_{n}(\omega)=-\log \max _{x \in I}\left|f_{\left.\omega\right|_{n}}^{\prime}(x)\right| ; \quad \tilde{\tau}_{k}(\omega)=\min \left\{n: \widetilde{S}_{n}(\omega) \geq k \chi\right\} .
\end{aligned}
$$

In the definitions above $n$ is a positive integer but $k$ is allowed to take positive non-integer values. $S_{n}$ will be shown to arise from a random walk driven by the derivative cocycle. Also, note that $\tilde{\tau}_{k}$ is a stopping time. Both $S_{n}$ and $\widetilde{S}_{n}$ are strictly increasing in $n$.

Definition 3.1. Let $X_{1}: \mathcal{A}^{\mathbb{N}} \rightarrow \mathbb{R}$ be the random variable

$$
X_{1}(\omega):=c\left(\omega_{1}, \sigma(\omega)\right)=-\log \left|f_{\omega_{1}}^{\prime}\left(x_{\sigma(\omega)}\right)\right| .
$$

For every integer $n>1$ we define

$$
X_{n}(\omega)=-\log \left|f_{\omega_{n}}^{\prime}\left(x_{\sigma^{n}\left(x_{\omega}\right)}\right)\right|=X_{1} \circ \sigma^{n-1} .
$$


Let $\theta$ be the law of the random variable $X_{1}$. Recall the definition of $D$ and $D^{\prime}$ from (41). Then for every $n, X_{n} \sim \theta$. Moreover, $\theta \in \mathcal{P}\left(\left[D, D^{\prime}\right]\right)$. In particular, the support of $\theta$ is bounded away from 0. These are immediate from Definition 3.1 and equation (44). The following Lemma is a direct consequence of the chain rule:

Lemma 3.2. For every $k>0$ and $\omega \in A^{\mathbb{N}}$ we have

$$
S_{n}(\omega)=\sum_{i=1}^{n} X_{i}(\omega)
$$

and

$$
-\log \left|f_{\left.\omega\right|_{\tau_{k}(\omega)}}^{\prime}\left(x_{\sigma^{\tau_{k}(\omega)}(\omega)}\right)\right|=S_{\tau_{k}(\omega)}(\omega) \in\left[k \chi, k \chi+D^{\prime}\right] .
$$

Next, we introduce some partitions of the space $\mathcal{A}^{\mathbb{N}}$ :

Definition 3.3. Given a finite word $\eta=\left(\eta_{1}, \cdots, \eta_{\ell}\right) \in \mathcal{A}^{\ell}$ :

1. Denote by $A_{\eta} \subseteq \mathcal{A}^{\mathbb{N}}$ the set of infinite words that begin with $\eta$,

$$
A_{\eta}:=\left\{\omega: \quad\left(\omega_{1}, \ldots, \omega_{\ell}\right)=\eta\right\} .
$$

2. For $h \in[0, \infty)$, let $\mathcal{A}^{h}$ be the partition of $\mathcal{A}^{\mathbb{N}}$ according to the function

$$
\iota^{h}: \omega \rightarrow\left(\omega_{1}, \cdots, \omega_{\tilde{\tau}_{h}(\omega)}\right) .
$$

This is a finite partition, and each set in it is a cylinder set of the form $A_{\eta}$.

3. Let $\eta^{\prime}$ be another finite word. We define the event

$$
A_{k, \eta, \eta^{\prime}}:=\left\{\omega \in A_{\eta}: \quad \sigma^{\tau_{k}(\omega)-1}(\omega) \in A_{\eta^{\prime}}\right\} .
$$

4. Given $k, h, h^{\prime} \geq 0$ we denote by $\mathcal{A}_{k}^{h, h^{\prime}}$ the finite partition of $\mathcal{A}^{\mathbb{N}}$ according to the map

$$
\iota_{k}^{h, h^{\prime}}=\left(\iota^{h}(\omega), \iota^{h^{\prime}}\left(\sigma^{\tau_{k}(\omega)-1}(\omega)\right) .\right.
$$

Note that every cell of the partition $\mathcal{A}_{k}^{h, h^{\prime}}$ has the form $A_{k, \eta, \eta^{\prime}}$.

Given $k, h, h^{\prime} \geq 0$ and $\omega \in \mathcal{A}^{\mathbb{N}}$ we write $\mathcal{A}_{k}^{h, h^{\prime}}(\omega)$ for the unique $\mathcal{A}_{k}^{h, h^{\prime}}$ cell that contains $\omega$. For $\mathbb{P}$-a.e. $\omega$, we denote the conditional measure of $\mathbb{P}$ on the corresponding cell by $\mathbb{P}_{\mathcal{A}_{k}^{h, h^{\prime}}(\omega)}$. Similarly, $\mathbb{P}_{\mathcal{A}^{h}(\omega)}$ denotes the conditional measure of $\mathbb{P}$ on the cylinder in the partition $\mathcal{A}^{h}$ that contains $\omega$. Recall that $\lambda$ is the Lebesgue measure on $\mathbb{R}$.

Definition 3.4. Let $k \in \mathbb{N}$ and let $\eta$, $\eta^{\prime}$ be finite words. Assuming $\mathbb{P}\left(A_{k, \eta, \eta^{\prime}}\right)>0$, we define a probability measure $\Gamma_{A_{k, \eta, \eta^{\prime}}}$ on $\left[k \chi, k \chi+D^{\prime}\right]$ by

$$
\Gamma_{A_{k, \eta, \eta^{\prime}}}:=\frac{\left.\int_{A_{\eta^{\prime}}} \lambda\right|_{\left[k \chi, k \chi+X_{1}\left(\omega^{\prime}\right)\right]} d \mathbb{P}\left(\omega^{\prime}\right)}{\int_{A_{\eta^{\prime}}} X_{1}\left(\omega^{\prime}\right) d \mathbb{P}\left(\omega^{\prime}\right)} .
$$

Note that there is actually no dependence on $\eta$; We use this notation for later convenience. 
Lemma 3.5. If $\mathbb{P}\left(A_{k, \eta, \eta^{\prime}}\right)>0$ then $\Gamma_{A_{k, \eta, \eta^{\prime}}} \ll \lambda_{\left[k \chi, k \chi+D^{\prime}\right]}$ with a density that depends only on $\mathbf{p}$, such that its norm is bounded above by $\frac{1}{D}$ independently of all parameters, including $k, \eta, \eta^{\prime}$.

Proof. We write $\Gamma$ instead of $\Gamma_{A_{k, \eta, \eta^{\prime}}}$. It is clear that $\Gamma \ll \lambda_{\left[k \chi, k \chi+D^{\prime}\right]}$. Next, assuming $x \in \operatorname{supp}(\Gamma)$, we need to bound

$$
\lim _{r \rightarrow 0} \frac{\Gamma(B(x, r))}{\lambda_{\left[k \chi, k \chi+D^{\prime}\right]}(B(x, r)}=\lim _{r \rightarrow 0} \frac{\Gamma(B(x, r))}{2 r}
$$

assuming $x$ is not an endpoint of $\left[k \chi, k \chi+D^{\prime}\right]$ (which we may assume). Then for every $\omega^{\prime} \in A_{k, \eta, \eta^{\prime}}$

$$
\lambda\left(\left[k \chi, k \chi+X_{1}\left(\omega^{\prime}\right)\right] \bigcap B(x, r)\right) \leq 2 r
$$

so

$$
\frac{\Gamma(B(x, r))}{2 r}=\frac{\int_{A_{\eta^{\prime}}} \lambda\left(\left[k \chi, k \chi+X_{1}\left(\omega^{\prime}\right)\right] \cap B(x, r)\right) d \mathbb{P}(\omega)}{2 r \cdot \mathbb{E}_{A_{k, \eta, \eta^{\prime}}}\left(X_{1}\right)} \leq \frac{1}{\mathbb{E}_{A_{k, \eta, \eta^{\prime}}}\left(X_{1}\right)} .
$$

Finally, by equation (4) we know that $X_{1}(\omega) \geq D$ for every $\omega$. We conclude that the density of $\Gamma$ is bounded by $\frac{1}{D}$, independently of all parameters.

Notation 3.6. We will use superscripts such as $o^{k \rightarrow \infty}(\cdot)$ in $O(\cdot)$ and $o(\cdot)$ to such bounds take place as which variables are being varied. The variables on which the implied constants depend on will be written in subscripts. The implied constant is absolute when no subscript is present.

The following local limit Theorem is one of the main keys to the proof of Theorem 1.1. It is the only place where the assumption $\Lambda_{c}=\{0\}$ from Theorem 1.1 is used.

Theorem 3.7. Fix $h_{0} \geq 0$. For all $k, h^{\prime}>0,0 \leq h \leq h_{0}$, and $A_{\eta} \in \mathcal{A}^{h}$, there exists a subset $\widetilde{A}_{k, \eta}^{h, h^{\prime}} \subseteq A_{\eta}$ such that:

(i) $\mathbb{P}\left(\widetilde{A}_{k, \eta}^{h, h^{\prime}}\right) \geq \mathbb{P}\left(A_{\eta}\right) \cdot\left(1-o_{h_{0}, \mathbf{p}}^{k \rightarrow \infty}(1)\right)$.

(ii) for all $\xi \in \widetilde{A}_{k, \eta}^{h, h^{\prime}}, \mathbb{P}\left(\mathcal{A}_{k}^{h, h^{\prime}}(\xi)\right)>0$.

(iii) for all $\xi \in \widetilde{A}_{k, \eta}^{h, h^{\prime}}$ and for any sub-interval $J \subseteq\left[k \chi, k \chi+D^{\prime}\right]$,

$$
\mathbb{P}_{\mathcal{A}_{k}^{h, h^{\prime}}(\xi)}\left(S_{\tau_{k}} \in J\right)=\Gamma_{\mathcal{A}_{k}^{h, h^{\prime}}(\xi)}(J)+o_{h_{0}, \mathbf{p}}^{k \rightarrow \infty}(1) .
$$

We emphasize that all bounds are uniform in $h^{\prime}$. We remark that to prove Theorem 1.1 part (1) we only need the case $h_{0}=0$ which means that $A_{\eta}$ is the full symbolic space. However, this more general version is needed to obtain the following upgraded version of Theorem 3.7, which is what we require for Theorem 1.1 part (2):

Theorem 3.8. For all $k, h^{\prime}, h>0$ and $A_{\eta} \in \mathcal{A}^{h}$, there exists a subset $\bar{A}_{k, \eta}^{h, h^{\prime}} \subseteq A_{\eta}$ such that:

(i) $\mathbb{P}\left(\bar{A}_{k, \eta}^{h, h^{\prime}}\right) \geq \mathbb{P}\left(A_{\eta}\right) \cdot\left(1-o_{\mathbf{p}}^{\min (h, k-h) \rightarrow \infty}(1)\right)$.

(ii) for all $\xi \in \bar{A}_{k, \eta}^{h, h^{\prime}}, \mathbb{P}\left(\mathcal{A}_{k}^{h, h^{\prime}}(\xi)\right)>0$.

\footnotetext{
${ }^{2}$ The referee has pointed out to us that this special case is related to the work of Kesten 35, Theorem 1]. Indeed, when $h_{0}=0$ one may deduce a weaker version of Part (iii) of Theorem 3.7 from Kesten's result.
} 
(iii) for all $\xi \in \bar{A}_{k, \eta}^{h, h^{\prime}}$ and for any sub-interval $J \subseteq\left[k \chi, k \chi+D^{\prime}\right]$,

$$
\mathbb{P}_{\mathcal{A}_{k}^{h, h^{\prime}(\xi)}}\left(S_{\tau_{k}} \in J\right)=\Gamma_{\mathcal{A}_{k}^{h, h^{\prime}}(\xi)}(J)+o_{\mathbf{p}}^{\min (h, k-h) \rightarrow \infty}(1) .
$$

The difference between Theorem 3.8 and Theorem 3.7 lies in the role of $h$ : In Theorem 3.7 it is assumed to be bounded by some uniform $h_{0}$. There is no such restriction in Theorem 3.8, but the "price" is that the error is now $o_{\mathbf{p}} \min (h, k-h) \rightarrow \infty(1)$ instead of $o_{h_{0}, \mathbf{p}}^{k \rightarrow \infty}(1)$. So, to make this error small, we need both $h$ and $k-h$ to go to $\infty$ simultaneously.

We proceed to prove Theorem 3.7. Afterwards, we prove Theorem 3.8 using the result of Theorem 3.7 as a black box and the $C^{1}$ linerization Lemma 2.4.

\subsection{Proof of Theorem 3.7}

\subsubsection{Benoist and Quint's central and local limit Theorems for cocycles with target}

The proof of Theorem 3.7 relies on two limit Theorems due to Benoist-Quint [3]. Before stating them, we need some preliminaries. First, notice that the indicator function $\mathbf{1}_{A_{\eta}}$ is a locally constant function on $\mathcal{A}^{\mathbb{N}}$. For the following Claim, recall the definition of the maps $\iota_{a}$ from Section 1.2.1 and the choice of our metric $d:=d_{\rho}$ on $\mathcal{A}^{\mathbb{N}}$ :

Claim 3.9. For every $a \in\{1, \ldots, n\}$ the following statements hold true:

1. The map $\iota_{a}$ is uniformly contracting:

$$
d\left(\iota_{a}(\omega), \iota_{a}(\eta)\right)=\rho d(\omega, \eta) .
$$

2. The cocycle $c(a, \omega)$ is uniformly bounded, Lipschitz in $\omega$, with a uniformly bounded Lipschitz constant as $a \in\{1, \ldots, n\}$ varies.

This is standard, since all the maps in $\Phi$ are $C^{1+\gamma}$ smooth and by equation (44). We are now ready to state a consequence of the central limit Theorem for cocycles with target, proved by Benoist-Quint:

Theorem 3.10. [3, Theorem 12.1. part (i)] Let $A_{\eta}$ be as in Theorem [3.7. There exists a variance $r=r(\boldsymbol{p})>0$ such that:

For every $R \in \mathbb{R}$ the function $\psi=1_{A_{\eta}} \times 1_{[R, \infty)}$ satisfies that

$$
\mathbb{P}\left(\omega \in A_{\eta}: \frac{\left|S_{n}(\omega)-n \cdot \chi\right|}{\sqrt{n}} \geq R\right)=\mathbb{P}\left(A_{\eta}\right)\left(N\left(0, r^{2}\right)>R\right)\left(1+o_{\psi}^{n \rightarrow \infty}(1)\right)
$$

where $\left(N\left(0, r^{2}\right)>R\right)$ stands for the probability that a Gaussian random variable with mean 0 and variance $r^{2}$ is larger than $R$.

We remark that [3, Theorem 12.1. part (i)] applies here since by Claim [3.9, the cocycle $c(\cdot, \cdot)$ satisfies the bounded moment conditions [3, (11.14),(11.15)] and is not constant. We also remark that for Theorem 3.10 we do not need the assumption that $\Lambda_{c}=\{0\}$ made in Theorem 1.1 . However, this assumption is crucial for the local limit Theorem for cocycles with target, also proved by Benoist-Quint: 
Theorem 3.11. [3, Theorem 16.15] Let $A_{\eta}$ be as in Theorem 3.7. Then for every $\omega^{\prime} \in \mathcal{A}^{\mathbb{N}}, \epsilon>0$, $m \in \mathbb{N}$ and $w \in \mathbb{R}_{+}$such that $|w-m \chi| \leq 4 \chi \sqrt{m \log m}$,

$$
\mathbb{P}_{\sigma^{-m}\left(\left\{\omega^{\prime}\right\}\right)}\left(\omega \in A_{\eta}, S_{m}(\omega) \in[w, w+\epsilon \chi]\right)=G_{\sqrt{m} r}(w-m \chi) \cdot \mathbb{P}\left(A_{\eta}\right) \cdot \epsilon \chi \cdot\left(1+o_{\epsilon, h_{0}, \boldsymbol{p}}^{m \rightarrow \infty}(1)\right)
$$

where $G_{s}(\cdot)$ stands for the density of the Gaussian law $N\left(0, s^{2}\right)$, and $r=r(\mathbf{p})$ is as in Theorem 3.10. The decay rate in $o_{\epsilon, h_{0}, p}^{m \rightarrow \infty}(1)$ depends only on $\epsilon \chi, h_{0}$ and $\boldsymbol{p}$, and is uniform in $\omega^{\prime}, w$.

To be precise, a-priori the decay rate depends only on $\epsilon \chi, A_{\eta}$ and $\mathbf{p}$. Hence it only depends on $\epsilon, h_{0}$, and $\mathbf{p}$ as there are only finitely many possible choices for $A_{\eta}$. In this case, as in Theorem 3.10. Claim 3.9 implies that our cocycle $c(\cdot, \cdot)$ satisfies the bounded moment conditions [3, (11.14),(11.15)]. It is aperiodic in the sense of [3, Equation (15.8)] by the assumption that $\Lambda_{c}=\{0\}$. By aperiodicity, the cocycle $\tilde{c}$ defined by [3, Equation (16.9)] is equal to $c$. So [3], Theorem 16.15] applies with, in the notations therein, $X=\mathcal{A}^{\mathbb{N}}, \varphi=\mathbf{1}_{A_{\eta}}$, the convex set $C$ being $[0, \epsilon \chi]$, the translation $v_{m}=w-m \chi$ and $v_{\mu}=0$, and finally $x=\omega^{\prime}$.

\subsubsection{Proof of Theorem 3.7}

For every $r \in \mathbb{R}$ let $U_{r}(x)=x+r$ be the translation map. In addition, for every $k$ we define the interval

$$
I_{k}=[k-\sqrt{k \log k}, k+\sqrt{k \log k}] .
$$

To begin the proof of Theorem 3.7, we decompose the left hand side in (iii) as

$$
\mathbb{P}_{\mathcal{A}_{k}^{h, h^{\prime}}(\xi)}\left(S_{\tau_{k}} \in J\right)=\sum_{m \notin I_{k}} \mathbb{P}_{\mathcal{A}_{k}^{h, h^{\prime}(\xi)}}\left(\tau_{k}=m+1, S_{\tau_{k}} \in J\right)+\sum_{m \in I_{k}} \mathbb{P}_{\mathcal{A}_{k}^{h, h^{\prime}}(\xi)}\left(\tau_{k}=m+1, S_{\tau_{k}} \in J\right) .
$$

The two terms are respectively treated by Proposition 3.13 and Proposition 3.12 below, and the Theorem follows.

Proposition 3.12. In the setting of Theorem 3.7, there exists a set $\widetilde{A}_{\eta}$ such that claims (i) and (ii) hold and for all $\xi \in \widetilde{A}_{\eta}$,

$$
\mathbb{P}_{\mathcal{A}_{k}^{h, h^{\prime}(\xi)}}\left(\tau_{k}-1 \notin I_{k}\right)=o_{h_{0}, \boldsymbol{p}}^{k \rightarrow \infty}(1)
$$

Notice that we are using the abbreviated notation $\widetilde{A}_{\eta}$ instead of $\widetilde{A}_{k, \eta}^{h, h^{\prime}}$.

Proof. We first prove the following claim:

$$
\mathbb{P}_{A_{\eta}}\left(\tau_{k}-1 \notin I_{k}\right)=o_{h_{0}, \mathbf{p}}^{k \rightarrow \infty}(1) \text {, for every } \eta \text {. }
$$

For the function $b=b(k)=\sqrt{k \log k}-1$, suppose that $\left|\tau_{k}(\omega)-k\right|>b=b(k)$. We also fix a small $\epsilon>0$. Without loss of generality, suppose first that $\tau_{k}(\omega)-k>b$. This implies that

$$
S_{\lfloor k+b\rfloor}(\omega)<k \chi
$$

and therefore

$$
\left|S_{\lfloor k+b\rfloor}(\omega)-\chi \cdot\lfloor k+b\rfloor\right| \geq\left|S_{\lfloor k+b\rfloor}(\omega)-k \chi-b \chi-\chi\right| \geq b \chi
$$


Let $r>0$ be as in Theorem [3.10, and let $R=R(r, \epsilon)>0$ be such that $\left(N\left(0, r^{2}\right)>R\right)=\epsilon$. Then by Theorem 3.10 applied for the corresponding $\psi$, we get

$$
\begin{aligned}
& \mathbb{P}\left(\omega \in A_{\eta}:\left|S_{\lfloor k+b\rfloor}(\omega)-\lfloor k+b\rfloor \chi\right| \geq b \chi\right) \\
= & \mathbb{P}\left(\omega \in A_{\eta}: \frac{\left|S_{\lfloor k+b\rfloor}(\omega)-\lfloor k+b\rfloor \chi\right|}{\sqrt{\lfloor k+b\rfloor}} \geq \frac{b \chi}{\sqrt{\lfloor k+b\rfloor}}\right) \\
\leq & \mathbb{P}\left(\omega \in A_{\eta}: \frac{\left|S_{\lfloor k+b\rfloor}(\omega)-\lfloor k+b\rfloor \chi\right|}{\sqrt{\lfloor k+b\rfloor}} \geq R\right) \\
= & \mathbb{P}\left(A_{\eta}\right)\left(N\left(0, r^{2}\right)>R\right)\left(1+o_{\psi}^{k \rightarrow \infty}(1)\right) \\
= & \epsilon+o_{\epsilon, h_{0}, \mathbf{p}}^{k \rightarrow \infty}(1) .
\end{aligned}
$$

Here we used that $\frac{b \chi}{\sqrt{\lfloor k+b\rfloor}} \rightarrow \infty$ as $k \rightarrow \infty$. Since $\epsilon$ is arbitrary, it follows that

$$
\mathbb{P}\left(\tau_{k}(\omega)-k>b\right) \leq \mathbb{P}\left(\left|S_{\lfloor k+b\rfloor}(\omega)-\lfloor k+b\rfloor \chi\right| \geq b \chi\right)=o_{h_{0}, \mathbf{p}}^{k \rightarrow \infty}(1) .
$$

A similar argument shows

$$
\mathbb{P}\left(\tau_{k}(\omega)-k<-b\right) \leq \mathbb{P}\left(\left|S_{\lceil k-b\rceil}(\omega)-\lceil k-b\rceil \chi\right| \geq b \chi\right)=o_{h_{0}, \mathbf{p}}^{k \rightarrow \infty}(1) .
$$

The claim (15) then follows by combining the two inequalities above.

The deduction of the proposition from (15) is standard. Indeed, it suffices to set

$$
\widetilde{A}_{\eta}=\left\{\xi \in A_{\eta}: \quad \mathbb{P}\left(\mathcal{A}_{k}^{h, h^{\prime}}(\xi)\right)>0, \quad \mathbb{P}_{\mathcal{A}_{k}^{h, h^{\prime}}(\xi)}\left(\tau_{k}-1 \notin I_{k}\right) \leq \sqrt{\mathbb{P}_{A_{\eta}}\left(\tau_{k}-1 \notin I_{k}\right)}\right\} .
$$

Then $\mathbb{P}_{A_{\eta}}\left(A_{\eta} \backslash \widetilde{A}_{\eta}\right) \leq \sqrt{\mathbb{P}_{A_{\eta}}\left(\tau_{k}-1 \notin I_{k}\right)}=o_{h_{0}, \mathbf{p}}^{k \rightarrow \infty}(1)$.

We now take care of the second term in (14):

Proposition 3.13. In the setting of Theorem [3.7, for all $\xi$ in the set $\widetilde{A}_{\eta}$ from Proposition 3.12,

$$
\sum_{m \in I_{k}} \mathbb{P}_{\mathcal{A}_{k}^{h, h^{\prime}(\xi)}}\left(\tau_{k}=m+1, S_{\tau_{k}} \in J\right)=\Gamma_{\mathcal{A}_{k}^{h, h^{\prime}}(\xi)}(J)+o_{h_{0}, \boldsymbol{p}}^{k \rightarrow \infty}(1)
$$

Proof. Let $\eta^{\prime}$ be the finite word such that $\mathcal{A}_{k}^{h, h^{\prime}}(\xi)=A_{k, \eta, \eta^{\prime}}$. We first notice that

$$
\begin{aligned}
& \sum_{m \in I_{k}} \mathbb{P}_{A_{k, \eta, \eta^{\prime}}}\left(\tau_{k}=m+1, S_{\tau_{k}} \in J\right) \\
= & \frac{\sum_{m \in I_{k}} \mathbb{P}\left(\omega \in A_{k, \eta, \eta^{\prime}}, \tau_{k}=m+1, S_{m+1} \in J\right)}{\mathbb{P}\left(A_{k, \eta, \eta^{\prime}}\right)} .
\end{aligned}
$$

Each summand in the numerator can be written as

$$
\begin{aligned}
& \mathbb{P}\left(\omega \in A_{k, \eta, \eta^{\prime}}, \quad \tau_{k}=m+1, \quad S_{m+1} \in J\right) \\
= & \mathbb{P}\left(\omega \in A_{\eta} \cap \sigma^{-m}\left(A_{\eta^{\prime}}\right), \quad S_{m}<k \chi, \quad S_{m+1} \in J\right) \\
= & \int_{A_{\eta^{\prime}}} \mathbb{P}_{\sigma^{-m}\left(\left\{\omega^{\prime}\right\}\right)}\left(\omega \in A_{\eta}, \quad S_{m}<k \chi, \quad S_{m+1} \in J\right) d \mathbb{P}\left(\sigma^{-m}\left(\left\{\omega^{\prime}\right\}\right)\right) \\
= & \int_{A_{\eta^{\prime}}^{k^{\prime}}} \mathbb{P}_{\sigma^{-m}\left(\left\{\omega^{\prime}\right\}\right)}\left(\omega \in A_{\eta}, \quad S_{m}<k \chi, \quad S_{m}+X_{1}\left(\omega^{\prime}\right) \in J\right) d \mathbb{P}\left(\omega^{\prime}\right) \\
= & \int_{A_{\eta^{\prime}}^{k^{\prime}}} \mathbb{P}_{\sigma^{-m}\left(\left\{\omega^{\prime}\right\}\right)}\left(\omega \in A_{\eta}, \quad S_{m} \in J^{\omega^{\prime}}\right) d \mathbb{P}\left(\omega^{\prime}\right)
\end{aligned}
$$


where the interval $J^{\omega^{\prime}}$ is defined by

$$
J^{\omega^{\prime}}:=\left[k \chi-X_{1}\left(\omega^{\prime}\right), k \chi\right) \cap U_{-X_{1}\left(\omega^{\prime}\right)} J .
$$

Fix an arbitrarily small $\epsilon>0$. Since $m \in I_{k}$ then by Theorem 3.11 for all translates $W \subseteq J^{\omega^{\prime}}$ of the form $[w, w+\epsilon \chi)$,

$$
\mathbb{P}_{\sigma^{-m}\left(\left\{\omega^{\prime}\right\}\right)}\left(\omega \in A_{\eta}, S_{m} \in W\right)=G_{\sqrt{m} r}(w-m \chi) \cdot \mathbb{P}\left(A_{\eta}\right) \cdot \epsilon \chi \cdot\left(1+o_{\epsilon, h_{0}, \mathbf{p}}^{m \rightarrow \infty}(1)\right)
$$

where we recall that $G_{s}(\cdot)$ stands for the density of $N\left(0, s^{2}\right), r>0$ is as in Theorem [3.10, and $o_{\epsilon, h_{0}, \mathbf{p}}^{k \rightarrow \infty}(1)$ in (19) depends only on $\epsilon, h_{0}$, and $\mathbf{p}$, and is uniform in $\omega^{\prime}$. Because $m \in I_{k}, m \rightarrow \infty$ if and only if $k \rightarrow \infty$, so we know by Lemma 3.14 below that

$$
\mathbb{P}_{\sigma^{-m}\left(\omega^{\prime}\right)}\left(\omega \in A_{\eta}, \quad S_{m} \in W\right)=G_{\sqrt{k} r}((m-k+\beta) \chi) \cdot \mathbb{P}\left(A_{\eta}\right) \cdot \epsilon \chi \cdot\left(1+o_{\epsilon, h_{0}, \mathbf{p}}^{k \rightarrow \infty}(1)\right)
$$

for all $\omega^{\prime} \in A_{\eta^{\prime}}, m \in I_{k}$ and $\beta \in[0,1)$ as $k \rightarrow \infty$.

Now, since the interval $J^{\omega^{\prime}}$ contains $\left\lfloor\frac{\lambda\left(J^{\omega^{\prime}}\right)}{\epsilon \chi}\right\rfloor$ disjoint intervals of the form $[w, w+\epsilon \chi)$ and is covered by $\left\lceil\frac{\lambda\left(J^{\omega^{\prime}}\right)}{\epsilon \chi}\right\rceil$ such intervals, we know that for all $m \in I_{k}$ and $\beta \in[0,1)$,

$$
\begin{aligned}
& \mathbb{P}_{\sigma^{-m}\left(\omega^{\prime}\right)}\left(\omega \in A_{\eta}, \quad S_{m} \in J^{\omega^{\prime}}\right) \\
= & G_{\sqrt{k} r}((m-k+\beta) \chi) \cdot \mathbb{P}\left(A_{\eta}\right) \cdot\left(\frac{\lambda\left(J^{\omega^{\prime}}\right)}{\epsilon \chi}+O(1)\right) \cdot \epsilon \chi \cdot\left(1+o_{\epsilon, h_{0}, \mathbf{p}}^{k \rightarrow \infty}(1)\right) \\
= & G_{\sqrt{k} r}((m-k+\beta) \chi) \cdot \mathbb{P}\left(A_{\eta}\right) \cdot\left(\lambda\left(J^{\omega^{\prime}}\right)+O(\epsilon \chi)+o_{\epsilon, h_{0}, \mathbf{p}}^{k \rightarrow \infty}(1)\right),
\end{aligned}
$$

where the implied constant in the $O(\epsilon \chi)$ is 1: the term represented by $O(\epsilon \chi)$ is of absolute value bounded by $\epsilon \chi$. The error term $o_{\epsilon, h_{0}, p}^{k \rightarrow \infty}(1)$ is uniform in $J, \omega^{\prime}$ and $\beta$.

Integrating (20) inside (17) leads to

$$
\begin{aligned}
& \mathbb{P}\left(\omega \in A_{k, \eta, \eta^{\prime}}, \quad \tau_{k}=m+1, \quad S_{m+1} \in J\right) \\
= & \int_{A_{\eta^{\prime}}} G_{\sqrt{k} r}((m-k+\beta) \chi) \cdot \mathbb{P}\left(A_{\eta}\right) \cdot\left(\lambda\left(J^{\omega^{\prime}}\right)+O(\epsilon \chi)+o_{\epsilon, h_{0}, \mathbf{p}}^{k \rightarrow \infty}(1)\right) d \mathbb{P}\left(\omega^{\prime}\right) \\
= & G_{\sqrt{k} r}((m-k+\beta) \chi) \cdot \mathbb{P}\left(A_{\eta}\right) \cdot \mathbb{P}\left(A_{\eta^{\prime}}\right) \cdot\left(\mathbb{E}_{\omega^{\prime} \in A_{\eta}^{\prime}}\left(\lambda\left(J^{\omega^{\prime}}\right)\right)+O(\epsilon \chi)+o_{\epsilon, h_{0}, \mathbf{p}}^{k \rightarrow \infty}(1)\right) .
\end{aligned}
$$

By summing over $m \in I_{k}$ and integrating over $\beta \in[0,1)$, we obtain

$$
\begin{aligned}
& \sum_{m \in I_{k}} \mathbb{P}\left(\omega \in A_{k, \eta, \eta^{\prime}}, \quad \tau_{k}=m+1, \quad S_{m+1} \in J\right) \\
= & \left(\int_{k-\sqrt{k \log k}}^{k+\sqrt{k \log k}+1} G_{\sqrt{k} r}((t-k) \chi) d t\right) \\
& \cdot \mathbb{P}\left(A_{\eta}\right) \mathbb{P}\left(A_{\eta^{\prime}}\right) \cdot\left(\mathbb{E}_{\omega^{\prime} \in A_{\eta}^{\prime}}\left(\lambda\left(J^{\omega^{\prime}}\right)\right)+O(\epsilon \chi)+o_{\epsilon, h_{0}, \mathbf{p}}^{k \rightarrow \infty}(1)\right) \\
= & \left(\int_{-\sqrt{k \log k}}^{\sqrt{k \log k}+1} G_{\sqrt{k} r}(t \chi) d t\right) \cdot \mathbb{P}\left(A_{\eta}\right) \mathbb{P}\left(A_{\eta^{\prime}}\right) \cdot\left(\mathbb{E}_{\omega^{\prime} \in A_{\eta}^{\prime}}\left(\lambda\left(J^{\omega^{\prime}}\right)\right)+O(\epsilon \chi)+o_{\epsilon, h_{0}, \mathbf{p}}^{k \rightarrow \infty}(1)\right) \\
= & \left(\int_{-\sqrt{\log k}}^{\sqrt{\log k}+\frac{1}{\sqrt{k}}} G_{r}(t \chi) d t\right) \cdot \mathbb{P}\left(A_{\eta}\right) \mathbb{P}\left(A_{\eta^{\prime}}\right) \cdot\left(\mathbb{E}_{\omega^{\prime} \in A_{\eta}^{\prime}}\left(\lambda\left(J^{\omega^{\prime}}\right)\right)+O(\epsilon \chi)+o_{\epsilon, h_{0}, \mathbf{p}}^{k \rightarrow \infty}(1)\right) \\
= & \left(\frac{1}{\chi}-o_{p}^{k \rightarrow \infty}(1)\right) \cdot \mathbb{P}\left(A_{\eta}\right) \mathbb{P}\left(A_{\eta^{\prime}}\right) \cdot\left(\mathbb{E}_{\omega^{\prime} \in A_{\eta}^{\prime}}\left(\lambda\left(J^{\omega^{\prime}}\right)\right)+O(\epsilon \chi)+o_{\epsilon, h_{0}, \mathbf{p}}^{k \rightarrow \infty}(1)\right) \\
= & \mathbb{P}\left(A_{\eta}\right) \mathbb{P}\left(A_{\eta^{\prime}}\right) \cdot\left(\frac{1}{\chi} \mathbb{E}_{\omega^{\prime} \in A_{\eta}^{\prime}}\left(\lambda\left(J^{\omega^{\prime}}\right)\right)+O(\epsilon)+o_{\epsilon, h_{0}, \mathbf{p}}^{k \rightarrow \infty}(1)\right)
\end{aligned}
$$


as $k \rightarrow \infty$. The term represented by $O(\epsilon)$ is uniformly bounded by $\epsilon$ in absolute value.

As $\epsilon>0$ is arbitrary, this implies that for all intervals $J \subset\left[k \chi, k \chi+D^{\prime}\right)$,

$$
\begin{aligned}
& \sum_{m \in I_{k}} \mathbb{P}\left(\omega \in A_{k, \eta, \eta^{\prime}}, \quad \tau_{k}=m+1, \quad S_{m+1} \in J\right) \\
= & \mathbb{P}\left(A_{\eta}\right) \mathbb{P}\left(A_{\eta^{\prime}}\right) \cdot\left(\frac{1}{\chi} \mathbb{E}_{\omega^{\prime} \in A_{\eta^{\prime}}}\left(\lambda\left(J^{\omega^{\prime}}\right)\right)+o_{h_{0}, \mathbf{p}}^{k \rightarrow \infty}(1)\right),
\end{aligned}
$$

where $J^{\omega^{\prime}}$ is defined by (18) and the error term $o_{h_{0}, \mathbf{p}}^{k \rightarrow \infty}(1)$ is uniform in $J, \omega^{\prime}$ and $\beta$.

Consider the special case of $J=\left[k \chi, k \chi+D^{\prime}\right)$, where $J^{\omega^{\prime}}=\left[k \chi-X_{1}\left(\omega^{\prime}\right), k \chi\right)$. Because the event $\left\{\tau_{k}=m+1, S_{\tau_{k}} \in\left[k \chi, k \chi+D^{\prime}\right)\right\}$ coincides with $\left\{\tau_{k}=m+1\right\}$, we obtain

$$
\begin{aligned}
& \sum_{m \in I_{k}} \mathbb{P}\left(\omega \in A_{k, \eta, \eta^{\prime}}, \tau_{k}=m+1\right) \\
= & \mathbb{P}\left(A_{\eta}\right) \mathbb{P}\left(A_{\eta^{\prime}}\right) \cdot\left(\frac{1}{\chi} \mathbb{E}_{\omega^{\prime} \in A_{\eta^{\prime}}}\left(\lambda\left(\left[k \chi-X_{1}\left(\omega^{\prime}\right), k \chi\right)\right)+o_{h_{0}, \mathbf{p}}^{k \rightarrow \infty}(1)\right)\right. \\
= & \mathbb{P}\left(A_{\eta}\right) \mathbb{P}\left(A_{\eta^{\prime}}\right) \cdot\left(\frac{1}{\chi} \mathbb{E}_{\omega^{\prime} \in A_{\eta^{\prime}}} X_{1}\left(\omega^{\prime}\right)+o_{h_{0}, \mathbf{p}}^{k \rightarrow \infty}(1)\right) .
\end{aligned}
$$

Therefore, by (16), (22) and (23),

$$
\begin{aligned}
& \sum_{m \in I_{k}} \mathbb{P}_{A_{k, \eta, \eta^{\prime}}}\left(\tau_{k}=m+1, S_{\tau_{k}} \in J\right) \\
& =\frac{\sum_{m \in I_{k}} \mathbb{P}\left(\omega \in A_{k, \eta, \eta^{\prime}}, \tau_{k}=m+1, S_{m+1} \in J\right)}{\sum_{m \in I_{k}} \mathbb{P}\left(\omega \in A_{k, \eta, \eta^{\prime}}, \tau_{k}=m+1\right)} \cdot \frac{\sum_{m \in I_{k}} \mathbb{P}\left(\omega \in A_{k, \eta, \eta^{\prime}}, \tau_{k}=m+1\right)}{\mathbb{P}\left(A_{k, \eta, \eta^{\prime}}\right)} \\
& =\frac{\frac{1}{\chi} \mathbb{E}_{\omega^{\prime} \in A_{\eta^{\prime}}}\left(\lambda\left(J^{\omega^{\prime}}\right)\right)+o_{h_{0}, p}^{k \rightarrow \infty}(1)}{\frac{1}{\chi} \mathbb{E}_{\omega^{\prime} \in A_{\eta^{\prime}}} X_{1}\left(\omega^{\prime}\right)+o_{h_{0}, \mathbf{p}}^{k \rightarrow \infty}(1)} \cdot \mathbb{P}_{A_{k, \eta, \eta^{\prime}}}\left(\tau_{k}-1 \in I_{k}\right) \\
& =\frac{\frac{1}{\chi} \mathbb{E}_{\omega^{\prime} \in A_{\eta^{\prime}}}\left(\lambda\left(J^{\omega^{\prime}}\right)\right)+o_{h_{0}, p}^{k \rightarrow \infty}(1)}{\frac{1}{\chi} \mathbb{E}_{\omega^{\prime} \in A_{\eta^{\prime}}} X_{1}\left(\omega^{\prime}\right)+o_{h_{0}, \mathbf{p}}^{k \rightarrow \infty}(1)} \cdot\left(1-o_{h_{0}, p}^{k \rightarrow \infty}(1)\right) \quad\left(\text { since } \xi \in \widetilde{A}_{\eta} \text { and } A_{k, \eta, \eta^{\prime}}=\mathcal{A}_{k}^{h, h^{\prime}}(\xi)\right) \\
& =\left(\frac{\mathbb{E}_{\omega^{\prime} \in A_{\eta^{\prime}}} \lambda\left(J^{\omega^{\prime}}\right)}{\mathbb{E}_{\omega^{\prime} \in A_{\eta^{\prime}}} X_{1}\left(\omega^{\prime}\right)}+o_{h_{0}, p}^{k \rightarrow \infty}(1)\right) \cdot\left(1-o_{h_{0}, \mathbf{p}}^{k \rightarrow \infty}(1)\right) \quad\left(\text { since } 0<D \leq \mathbb{E}_{\omega^{\prime} \in A_{\eta^{\prime}}} X_{1}\left(\omega^{\prime}\right) \leq D^{\prime}\right) \\
& =\frac{\mathbb{E}_{\omega^{\prime} \in A_{\eta}^{\prime}} \lambda\left(J^{\omega^{\prime}}\right)}{\mathbb{E}_{\omega^{\prime} \in A_{\eta^{\prime}}} X_{1}\left(\omega^{\prime}\right)}+o_{h_{0}, \mathbf{p}}^{k \rightarrow \infty}(1) \text {. }
\end{aligned}
$$

To conclude, it suffices to notice that $\frac{\mathbb{E}_{\omega^{\prime} \in A_{\eta^{\prime}}} \lambda\left(J^{\omega^{\prime}}\right)}{\mathbb{E}_{\omega^{\prime} \in A_{\eta^{\prime}}} X_{1}\left(\omega^{\prime}\right)}$ is exactly $\Gamma_{A_{k, \eta, \eta^{\prime}}}(J)$.

Lemma 3.14. For $m \in I_{k}, w \in\left[k \chi-D^{\prime}, k \chi\right]$ and $\beta \in[0,1)$,

$$
\left|\frac{G_{\sqrt{m} r}(w-m \chi)}{G_{\sqrt{k} r}((m-k+\beta) \chi)}-1\right| \lesssim_{\mathbf{p}} k^{-\frac{1}{2}}(\log k)^{\frac{3}{2}} \text { as } k \rightarrow \infty,
$$

Proof. Recall $G_{s}(x)=\frac{1}{\sqrt{2 \pi} s} \exp \left(-\frac{x^{2}}{2 s^{2}}\right)$ and $\log G_{s}(x)=-\log \sqrt{2 \pi}-\log s-\frac{x^{2}}{2 s^{2}}$. 
So as $k \rightarrow \infty$,

$$
\begin{aligned}
& \left|\log G_{\sqrt{m} r}(w-m \chi)-\log G_{\sqrt{k} r}(w-m \chi)\right| \\
\leq & |\log (\sqrt{m} r)-\log (\sqrt{k} r)|+\frac{(w-m \chi)^{2}}{2 r^{2}}\left|\frac{1}{m}-\frac{1}{k}\right| \\
\leq & \frac{1}{2}\left|\log \frac{m}{k}\right|+\frac{\left(|k-m| \chi+D^{\prime}\right)^{2}}{2 m r^{2}}\left|\frac{m}{k}-1\right| \\
\lesssim & \left|\frac{m}{k}-1\right|+\frac{(\sqrt{k \log k}+1)^{2}}{k}\left|\frac{m}{k}-1\right| \lesssim(\log k)\left|\frac{m}{k}-1\right| \\
\lesssim & (\log k) \frac{\sqrt{\log k}}{\sqrt{k}}=k^{-\frac{1}{2}}(\log k)^{\frac{3}{2}}
\end{aligned}
$$

where the implied constant depends only on $\chi, D^{\prime}$ and $r$, and hence only on $p$.

Moreover,

$$
\begin{aligned}
&\left|\log G_{\sqrt{k} r}(w-m \chi)-\log G_{\sqrt{k} r}((m-k+\beta) \chi)\right| \\
&= \frac{1}{2 k r^{2}}\left|(w-m \chi)^{2}-(k \chi-m \chi+\beta \chi)^{2}\right| \\
&= \frac{1}{2 k r^{2}} \cdot|w-k \chi-\beta \chi| \cdot|2(k-m-\beta) \chi+(w-k \chi-\beta \chi)| \\
& \leq \frac{1}{2 k r^{2}} \cdot\left(D^{\prime}+\beta \chi\right) \cdot\left(2(\sqrt{k \log k}+1) \chi+\left(D^{\prime}+\beta \chi\right)\right) \\
& \lesssim k^{-\frac{1}{2}}(\log k)^{\frac{1}{2}}
\end{aligned}
$$

where the implied constant similarly depends only on $p$.

Combining the two inequalities above shows

$$
\left|\log G_{\sqrt{m} r}(w-m \chi)-\log G_{\sqrt{k} r}((m-k+\beta) \chi)\right| \lesssim k^{-\frac{1}{2}}(\log k)^{\frac{3}{2}},
$$

which in turn implies the lemma.

\subsection{Proof of Theorem 3.8}

\subsubsection{Fixing parameters and preliminary steps}

Fix $\epsilon>0$ and choose $\ell=\ell(\epsilon, \mathbf{p})$ such that $e^{-\ell \chi}<\epsilon^{\frac{2}{\gamma}}$. In this proof, we will view $\epsilon, \mathbf{p}$ and $\ell$ as fixed inputs, while $k$ and $h$ are varying.

Suppose

$$
\min (h, k-h)>\ell
$$

and fix $A_{\eta} \in \mathcal{A}^{h}$. Decompose $\eta=\eta^{\#} \eta^{*}$ where $A_{\eta^{\#}} \in \mathcal{A}^{h-\ell}$. Then

$$
-\log \max _{x \in I}\left|f_{\eta^{*}}^{\prime}(x)\right| \in\left[\ell \chi-O_{\mathbf{p}}(1), \ell \chi+O_{\mathbf{p}}(1)\right]
$$

by bounded distortion (Theorem 2.1). Define real values $h^{*}=h^{*}(\epsilon, \mathbf{p}, \eta, h)$ and $k^{*}=k^{*}(\epsilon, \mathbf{p}, \eta, h, k)$ by

$$
\begin{gathered}
h^{*}=\frac{-\log \max _{x \in I}\left|f_{\eta^{*}}^{\prime}(x)\right|}{\chi} \in\left[\ell-O_{\mathbf{p}}(1), \ell+O_{\mathbf{p}}(1)\right], \\
k^{*}=k+\frac{\log \max _{x \in I}\left|f_{\eta^{\#}}^{\prime}\left(f_{\eta^{*}}(x)\right)\right|-C \epsilon^{2}}{\chi} \in\left[k-h+\ell-O_{\mathbf{p}}(1), k-h+\ell+O_{\mathbf{p}}(1)\right],
\end{gathered}
$$

where $C=C(\mathbf{p})$ stands for the implied constant in Lemma 3.15 below. 
It is important that $h^{*}$ is uniformly bounded even though it depends on $\eta$ and $h$. It follows that $k^{*}-(k-h)=O_{\epsilon, \mathbf{p}}(1)$, and that $k^{*}<k$ if $h>O_{\epsilon, \mathbf{p}}(1)$.

We will keep the value $h^{\prime}$.

Note that $A_{\eta}=\eta^{\#} \cdot A_{\eta^{*}}$, where we use the notation

$$
\theta \cdot Y=\left\{\theta \theta^{*}: \theta^{*} \in Y\right\}
$$

Moreover, by the choice of $h^{*}, A_{\eta^{*}} \in \mathcal{A}^{h^{*}}$.

Lemma 3.15. For all $\xi^{*} \in A_{\eta^{*}}$

$$
0 \leq \log \max _{x \in I}\left|f_{\eta^{\#}}^{\prime}\left(f_{\eta^{*}}(x)\right)\right|-\log \left|f_{\eta^{\#}}^{\prime}\left(x_{\xi^{*}}\right)\right| \lesssim_{\mathbf{p}} \epsilon^{2} .
$$

We denote the implied constant by $C=C(\mathbf{p})$.

Proof. The inequality $0 \leq$ is obvious because of the max. For the second inequality, since $x_{\xi^{*}}=$ $f_{\eta^{*}}\left(x_{\sigma^{\left|\eta^{*}\right| \xi^{*}}}\right)$, by bounded distortion (Theorem 2.1),

$$
\left|x_{\xi^{*}}-f_{\eta^{*}}(x)\right| \leq\left|f_{\eta^{*}}(I)\right| \lesssim_{\mathbf{p}} e^{-\ell \chi}<\epsilon^{\frac{2}{\gamma}}
$$

The Lemma follows by an application of Lemma 2.4.

\subsubsection{Specifying the exceptional set $\bar{A}_{k, \eta}^{h, h^{\prime}}$}

Let $\widetilde{A}_{k^{*}, \eta^{*}}^{h^{*}}$ be given by Theorem 3.7, for the corresponding parameters as in Section 3.3.1 (here it is important that $h^{*}$ is uniformly bounded). For clarity, we repeat the properties satisfied by this set:

$\left(i^{*}\right) \mathbb{P}\left(\widetilde{A}_{k^{*}, \eta^{*}}^{h^{*}, h^{\prime}}\right) \geq \mathbb{P}\left(A_{\eta^{*}}\right) \cdot\left(1-o_{\ell+O_{\mathbf{p}}(1), \mathbf{p}}^{k^{*} \rightarrow \infty}(1)\right)=\mathbb{P}\left(A_{\eta^{*}}\right) \cdot\left(1-o_{\epsilon, \mathbf{p}}^{k-h \rightarrow \infty}(1)\right)$.

(ii $\left.{ }^{*}\right)$ For all $\omega^{*} \in \widetilde{A}_{k^{*}, \eta^{*}}^{h^{*}, h^{\prime}}, \mathbb{P}\left(\mathcal{A}_{k^{*}}^{h^{*}, h^{\prime}}\left(\omega^{*}\right)\right)>0$.

(iii*) For all $\omega^{*} \in \widetilde{A}_{k^{*}, \eta^{*}}^{h^{*}, h^{\prime}}$ and for any sub-interval $J \subseteq\left[k \chi, k \chi+D^{\prime}\right]$,

$$
\mathbb{P}_{\mathcal{A}_{k^{*}}^{h^{*}, h^{\prime}}\left(\omega^{*}\right)}\left(S_{\tau_{k^{*}}} \in J\right)=\Gamma_{\mathcal{A}_{k^{*}}^{h^{*}, h^{\prime}}\left(\omega^{*}\right)}(J)+o_{\epsilon, \mathbf{p}}^{k-h \rightarrow \infty}(1) .
$$

Define an exceptional subset by

$$
Z_{k, \eta}^{h, h^{\prime}}=\left\{\xi^{*} \in A_{\eta^{*}}: \quad \tau_{k}\left(\eta^{\#} \xi^{*}\right) \neq\left|\eta^{\#}\right|+\tau_{k^{*}}\left(\xi^{*}\right)\right\} .
$$

Lemma 3.16. $\mathbb{P}\left(Z_{k, \eta}^{h, h^{\prime}}\right) \leq \mathbb{P}\left(A_{\eta^{*}}\right) \cdot\left(O_{\mathbf{p}}\left(\epsilon^{2}\right)+o_{\epsilon, \mathbf{p}}^{k-h \rightarrow \infty}(1)\right)$.

Proof. We need to show $\mathbb{P}_{A_{\eta^{*}}}\left(Z_{k, \eta}^{h, h^{\prime}}\right) \leq O_{\mathbf{p}}\left(\epsilon^{2}\right)+o_{\epsilon, \mathbf{p}}^{k-h \rightarrow \infty}(1)$. Since $\mathbb{P}_{A_{\eta^{*}}}\left(\widetilde{A}_{k^{*}, \eta^{*}}^{h^{*}, h^{\prime}}\right) \geq 1-o_{\epsilon, \mathbf{p}}^{k-h \rightarrow \infty}(1)$, it suffices to show $\mathbb{P}_{A_{\eta^{*}}}\left(\widetilde{A}_{k^{*}, \eta^{*}}^{h^{*}, h^{\prime}} \cap Z_{k, \eta}^{h, h^{\prime}}\right) \leq O_{\mathbf{p}}\left(\epsilon^{2}\right)+o_{\epsilon, \mathbf{p}}^{k-h \rightarrow \infty}(1)$.

Now, as $A_{\eta^{*}}$ is $\mathcal{A}_{k^{*}}^{h^{*}, h^{\prime}}$-measurable, it suffices to show that for every $\omega^{*} \in \widetilde{A}_{k^{*}, \eta^{*}}^{h^{*}, h^{\prime}}$,

$$
\mathbb{P}_{\mathcal{A}_{k^{*}}^{h^{*}, h^{\prime}}\left(\omega^{*}\right)}\left(\widetilde{A}_{k^{*}, \eta^{*}}^{h^{*}, h^{\prime}} \cap Z_{k, \eta}^{h, h^{\prime}}\right) \leq O_{\mathbf{p}}\left(\epsilon^{2}\right)+o_{\epsilon, \mathbf{p}}^{k-h \rightarrow \infty}(1)
$$


Suppose $\xi^{*} \in \widetilde{A}_{k^{*}, \eta^{*}}^{h^{*}, h^{\prime}} \cap Z_{k, \eta}^{h, h^{\prime}}$. For simplicity write $a=\left|\eta^{\#}\right|$ and $b=\tau_{k^{*}}\left(\xi^{*}\right)$, then $S_{b-1}\left(\xi^{*}\right)<$ $k^{*} \leq S_{b}\left(\xi^{*}\right)$. Since $\tau_{k}\left(\eta^{\#} \xi^{*}\right) \neq a+b$ then one of the following holds:

Case 1: $S_{a+b-1}\left(\eta^{\#} \xi^{*}\right) \geq k$, which implies

$$
\begin{aligned}
\frac{-\log \left|f_{\eta^{\#}}^{\prime}\left(x_{\xi^{*}}\right)\right|}{\chi} & =S_{a}\left(\eta^{\#} \xi^{*}\right)=S_{a+b-1}\left(\eta^{\#} \xi^{*}\right)-S_{b-1}\left(\xi^{*}\right) \\
& >k-k^{*}=\frac{-\log \max _{x \in I}\left|f_{\eta^{\#}}^{\prime}\left(f_{\eta^{*}}(x)\right)\right|+C \epsilon^{2}}{\chi} .
\end{aligned}
$$

Comparing with Lemma 3.15, we know this case cannot happen.

Case 2: $S_{a+b}\left(\eta^{\#} \xi^{*}\right)<k$, which implies

$$
\begin{aligned}
\frac{-\log \left|f_{\eta^{\#}}^{\prime}\left(x_{\xi^{*}}\right)\right|}{\chi} & =S_{a}\left(\eta^{\#} \xi^{*}\right)=S_{a+b}\left(\eta^{\#} \xi^{*}\right)-S_{b}\left(\xi^{*}\right) \\
& <k-k^{*}=\frac{-\log \max _{x \in I}\left|f_{\eta^{\#}}^{\prime}\left(f_{\eta^{*}}(x)\right)\right|+C \epsilon^{2}}{\chi} .
\end{aligned}
$$

Comparing with Lemma 3.15, we know $S_{b}\left(\xi^{*}\right) \in\left[k^{*}, k^{*}+C \epsilon^{2}\right)$. Since $\Gamma_{A_{k^{*}, \eta^{*}, \eta^{\prime}}}$ is absolutely continuous with a uniformly bounded density (Lemma 3.5),

$$
\Gamma_{A_{k^{*}, \eta^{*}, \eta^{\prime}}}\left(\left[k^{*}, k^{*}+C \epsilon^{2}\right)\right) \lesssim_{\mathbf{p}} \epsilon^{2}
$$

uniformly. The lemma follows from property (iii*) of the set $\widetilde{A}_{k^{*}, \eta^{*}}^{h^{*}}, h^{\prime}$

Next, set

$$
\begin{aligned}
\widetilde{Z}_{k, \eta}^{h, h^{\prime}}=\left\{\omega \in A_{\eta}: \quad \mathbb{P}_{\mathcal{A}_{k}^{h, h^{\prime}}(\omega)}\left(\eta^{\#} \cdot Z_{k, \eta}^{h, h^{\prime}}\right) \geq \mathbb{P}_{\mathcal{A}_{\eta^{*}}}\left(Z_{k, \eta}^{h, h^{\prime}}\right)^{\frac{1}{2}}\right\} \\
\cup \eta^{\#} \cdot\left\{\omega^{*} \in A_{\eta^{*}}: \quad \mathbb{P}_{\mathcal{A}_{k^{*}}^{h^{*} h^{\prime}}\left(\omega^{*}\right)}\left(Z_{k, \eta}^{h, h^{\prime}}\right) \geq \mathbb{P}_{\mathcal{A}_{\eta^{*}}}\left(Z_{k, \eta}^{h, h^{\prime}}\right)^{\frac{1}{2}}\right\} .
\end{aligned}
$$

Because $\mathbb{P}_{A_{\eta}}\left(\eta^{\#} \cdot Z_{k, \eta}^{h, h^{\prime}}\right)=\mathbb{P}_{A_{\eta^{*}}}\left(Z_{k, \eta}^{h, h^{\prime}}\right)$ and $\eta^{\#}$. sends $\mathbb{P}_{\eta^{*}}$ to $\mathbb{P}_{\eta}$, we have

$$
\mathbb{P}_{A_{\eta}}\left(\widetilde{Z}_{k, \eta}^{h, h^{\prime}}\right) \leq \mathbb{P}_{A_{\eta^{*}}}\left(Z_{k, \eta}^{h, h^{\prime}}\right)^{\frac{1}{2}}=O_{\mathbf{p}}(\epsilon)+o_{\epsilon, \mathbf{p}}^{k-h \rightarrow \infty}(1),
$$

since this bound holds for both components in $\widetilde{Z}_{k, \eta}^{h, h^{\prime}}$.

The set $\bar{A}_{k, \eta}^{h, h^{\prime}}$ can be now defined as

$$
\bar{A}_{k, \eta}^{h, h^{\prime}}=\left(\eta^{\#} \cdot\left(\widetilde{A}_{k^{*}, \eta^{*}}^{h^{*}, h^{\prime}} \backslash Z_{k, \eta}^{h, h^{\prime}}\right)\right) \backslash \widetilde{Z}_{k, \eta}^{h, h^{\prime}} .
$$

\subsubsection{Proof of Theorem 3.8}

For property (i), it suffices to prove that if $\min (h, k-h)$ is sufficiently large (depending only on $\epsilon$ and $\mathbf{p})$, then $\frac{\mathbb{P}\left(\bar{A}_{k, \eta}^{h, h^{\prime}}\right)}{\mathbb{P}\left(A_{\eta}\right)}>1-O_{\mathbf{p}}(\epsilon)$. Indeed,

$$
\begin{aligned}
\frac{\mathbb{P}\left(\bar{A}_{k, \eta}^{h, h^{\prime}}\right)}{\mathbb{P}\left(A_{\eta}\right)} & =\frac{\mathbb{P}\left(\widetilde{A}_{k^{*}, \eta^{*}}^{h^{*}, h^{\prime}} \backslash Z_{k, \eta}^{h, h^{\prime}}\right)}{\mathbb{P}\left(A_{\eta^{*}}\right)}-\frac{\mathbb{P}\left(\widetilde{Z}_{k, \eta}^{h, h^{\prime}}\right)}{\mathbb{P}\left(A_{\eta}\right)} \\
& \geq\left(\left(1-o_{\mathbf{p}}^{k-h \rightarrow \infty}(1)\right)-\left(O_{\mathbf{p}}\left(\epsilon^{2}\right)+o_{\epsilon, \mathbf{p}}^{k-h \rightarrow \infty}(1)\right)\right)-\left(O_{\mathbf{p}}(\epsilon)+o_{\epsilon, \mathbf{p}}^{k-h \rightarrow \infty}(1)\right) \\
& =1-\left(O_{\mathbf{p}}(\epsilon)+o_{\epsilon, \mathbf{p}}^{k-h \rightarrow \infty}(1)\right) .
\end{aligned}
$$


This implies the claim.

For property (ii): This property is a formality and always holds after omitting a null set (in fact, we don't need to omit a null set based on the construction here).

The proof of property (iii) will requires more effort. By adjusting the threshold on $\min (h, k-h)$ based on $\epsilon$, it suffices to prove that

$$
\mathbb{P}_{\mathcal{A}_{k}^{h, h^{\prime}}(\omega)}\left(S_{\tau_{k}} \in J\right)=\Gamma_{\mathcal{A}_{k}^{h, h^{\prime}}(\omega)}(J)+O_{p}(\epsilon)+o_{\epsilon, \mathbf{p}}^{\min (h, k-h) \rightarrow \infty}(1)
$$

for all $\omega \in \bar{A}_{k, \eta}^{h, h^{\prime}}$. In fact, proving $\geq$ instead of $=$ is enough, by applying to both $J$ and $J^{c}$.

In particular, it suffices to show

$$
\mathbb{P}_{\mathcal{A}_{k}^{h, h^{\prime}(\omega)}}\left(\left\{\xi \in \eta^{\#} \cdot\left\{A_{\eta^{*}} \backslash Z_{k, \eta}^{h, h^{\prime}}\right\}: \quad S_{\tau_{k}(\xi)} \in J\right\}\right) \geq \Gamma_{\mathcal{A}_{k}^{h, h^{\prime}}(\omega)}(J)-O_{p}(\epsilon)-o_{\epsilon, \mathbf{p}}^{k-h \rightarrow \infty}(1) .
$$

In the following Lemmas we take a closer look at $\eta^{\#} \cdot\left\{A_{\eta^{*}} \backslash Z_{k, \eta}^{h, h^{\prime}}\right\}$ :

Lemma 3.17. If $\omega \in \bar{A}_{k, \eta}^{h, h^{\prime}}$, then

$$
\mathcal{A}_{k}^{h, h^{\prime}}(\omega) \backslash\left(\eta^{\#} \cdot Z_{k, \eta}^{h, h^{\prime}}\right)=\eta^{\#} \cdot\left(\mathcal{A}_{k^{*}}^{h^{*}, h^{\prime}}\left(\omega^{*}\right) \backslash Z_{k, \eta}^{h, h^{\prime}}\right) .
$$

Proof. The $\subseteq$ direction: Suppose $\xi \in \mathcal{A}_{k}^{h, h^{\prime}}(\omega) \backslash\left(\eta^{\#} \cdot Z_{k, \eta}^{h, h^{\prime}}\right)$, then both $\xi, \omega \in \eta^{\#} \cdot\left(A_{\eta^{*}} \backslash Z_{k, \eta}^{h, h^{\prime}}\right)$. Write them respectively as $\eta^{\#} \xi^{*}$ and $\eta^{\#} \omega^{*}$, then

$$
\tau_{k}(\xi)-\tau_{k^{*}}\left(\xi^{*}\right)=\left|\eta^{\#}\right|=\tau_{k}(\omega)-\tau_{k^{*}}\left(\omega^{*}\right) .
$$

Hence,

$$
\sigma^{\tau_{k^{*}}\left(\xi^{*}\right)} \xi^{*}=\sigma^{\tau_{k}(\xi)} \xi \equiv_{\mathcal{A}^{h^{\prime}}} \sigma^{\tau_{k}(\omega)} \omega=\sigma^{\tau_{k^{*}}\left(\omega^{*}\right)} \omega^{*} .
$$

This shows that $\xi^{*} \in \mathcal{A}_{k^{*}}^{h^{*} h^{\prime}}\left(\omega^{*}\right)$, meaning $\mathcal{A}_{k}^{h, h^{\prime}}(\omega) \backslash\left(\eta^{\#} \cdot Z_{k, \eta}^{h, h^{\prime}}\right) \subseteq \eta^{\#} \cdot \mathcal{A}_{k^{*}}^{h^{*}, h^{\prime}}\left(\omega^{*}\right)$. In fact:

$$
\mathcal{A}_{k}^{h, h^{\prime}}(\omega) \backslash\left(\eta^{\#} \cdot Z_{k, \eta}^{h, h^{\prime}}\right) \subseteq \eta^{\#} \cdot\left(\mathcal{A}_{k^{*}}^{h^{*}, h^{\prime}}\left(\omega^{*}\right) \backslash Z_{k, \eta}^{h, h^{\prime}}\right) .
$$

The $\supseteq$ direction: Keep the notations and assume $\xi^{*} \in \mathcal{A}_{k^{*}}^{h^{*}, h^{\prime}}\left(\omega^{*}\right) \backslash Z_{k, \eta}^{h, h^{\prime}}$. The equality (26) still holds, showing that

$$
\sigma^{\tau_{k}(\xi)} \xi=\sigma^{\tau_{k^{*}}\left(\xi^{*}\right)} \xi^{*} \equiv_{\mathcal{A}^{h^{\prime}}} \sigma^{\tau_{k^{*}}\left(\omega^{*}\right)} \omega^{*}=\sigma^{\tau_{k}(\omega)} \omega
$$

Hence $\eta^{\#} \cdot\left(\mathcal{A}_{k^{*}}^{h^{*} h^{\prime}}\left(\omega^{*}\right) \backslash Z_{k, \eta}^{h, h^{\prime}}\right) \subseteq \mathcal{A}_{k}^{h, h^{\prime}}(\omega)$, or more precisely,

$$
\eta^{\#} \cdot\left(\mathcal{A}_{k^{*}}^{h^{*}, h^{\prime}}\left(\omega^{*}\right) \backslash Z_{k, \eta}^{h, h^{\prime}}\right) \subseteq \mathcal{A}_{k}^{h, h^{\prime}}(\omega) \backslash\left(\eta^{\#} \cdot Z_{k, \eta}^{h, h^{\prime}}\right) .
$$

Lemma 3.18. If $\omega=\eta^{\#} \omega^{*} \in \bar{A}_{k, \eta}^{h, h^{\prime}}$ and $\xi=\eta^{\#} \xi^{*} \in \mathcal{A}_{k}^{h, h^{\prime}}(\omega) \backslash\left(\eta^{\#} \cdot Z_{k, \eta}^{h, h^{\prime}}\right)$, then

$$
S_{\tau_{k}}(\xi)-S_{\tau_{k^{*}}}\left(\xi^{*}\right) \in\left[k-k^{*}-\frac{C \epsilon^{2}}{\chi}, k-k^{*}\right]
$$

where $C$ is as in Lemma 3.15 . 
Proof. By the proof of the previous lemma,

$$
\begin{aligned}
& S_{\tau_{k}}(\xi)-S_{\tau_{k^{*}}}\left(\xi^{*}\right) \\
= & S_{\mid \eta^{\# \mid}}(\xi)=\frac{-\log \left|f_{\eta^{\#}}^{\prime}\left(x_{\xi^{*}}\right)\right|}{\chi} \\
\in & {\left[\frac{-\log \max _{x \in I}\left|f_{\eta^{\#}}^{\prime}\left(f_{\eta^{*}}(x)\right)\right|}{\chi}, \frac{-\log \max _{x \in I}\left|f_{\eta^{\#}}^{\prime}\left(f_{\eta^{*}}(x)\right)\right|+C \epsilon^{2}}{\chi}\right] } \\
= & {\left[k-k^{*}-\frac{C \epsilon^{2}}{\chi}, k-k^{*}\right] . }
\end{aligned}
$$

We proceed to compute a few auxiliary bounds:

Since $\omega \notin \widetilde{Z}_{k, \eta}^{h, h^{\prime}}$

$$
\mathbb{P}_{\mathcal{A}_{k}^{h, h^{\prime}(\omega)}}\left(\mathcal{A}_{k}^{h, h^{\prime}}(\omega) \backslash\left(\eta^{\#} \cdot Z_{k, \eta}^{h, h^{\prime}}\right)\right) \geq 1-\mathbb{P}_{A_{\eta^{*}}}\left(Z_{k, \eta}^{h, h^{\prime}}\right)^{\frac{1}{2}} \geq 1-O_{\mathbf{p}}(\epsilon)-o_{\epsilon, \mathbf{p}}^{k \rightarrow \infty}(1) .
$$

Moreover, by Lemma 3.17, Lemma 3.18 and property (iii*).

$$
\begin{aligned}
& \mathbb{P}_{\mathcal{A}_{k}^{h, h^{\prime}}(\omega) \backslash\left(\eta^{\#} \cdot Z_{k, \eta}^{h, h^{\prime}}\right)}\left(\left\{\xi \in \mathcal{A}_{k}^{h, h^{\prime}}(\omega) \backslash\left(\eta^{\#} \cdot Z_{k, \eta}^{h, h^{\prime}}\right): S_{\tau_{k}(\xi)} \in J\right\}\right) \\
& \geq \mathbb{P}_{\eta^{\# \cdot} \cdot \mathcal{A}_{k^{*}}^{h^{*}, h^{\prime}}\left(\omega^{*}\right)}\left(\left\{\xi \in \mathcal{A}_{k}^{h, h^{\prime}}(\omega) \backslash\left(\eta^{\#} \cdot Z_{k, \eta}^{h, h^{\prime}}\right): S_{\tau_{k}(\xi)} \in J\right\}\right)
\end{aligned}
$$

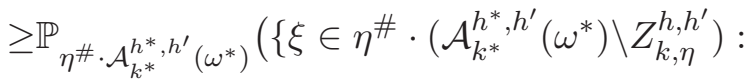

$$
\begin{aligned}
& \left.\left.S_{\tau_{k^{*}}\left(\xi^{*}\right)} \in\left(J-\left(k-k^{*}-\frac{C \epsilon^{2}}{\chi}\right)\right) \cap\left(J-\left(k-k^{*}\right)\right)\right\}\right) \\
& \geq \mathbb{P}_{\mathcal{A}_{k^{*}}^{h^{*}, h^{\prime}}\left(\omega^{*}\right)}\left(S_{\tau_{k^{*}}} \in J^{*}\right)-\mathbb{P}_{\mathcal{A}_{k^{*}}^{h^{*}, h^{\prime}}\left(\omega^{*}\right)}\left(Z_{k, \eta}^{h, h^{\prime}}\right) \\
& \geq\left(\Gamma_{\mathcal{A}_{k^{*}}^{h^{*}, h^{\prime}}\left(\omega^{*}\right)}\left(J^{*}\right)-o_{\epsilon, \mathbf{p}}^{k-h \rightarrow \infty}(1)\right)-\mathbb{P}_{A_{\eta^{*}}}\left(Z_{k, \eta}^{h, h^{\prime}}\right)^{\frac{1}{2}} \\
& \geq \Gamma_{\mathcal{A}_{k^{*}}^{h^{*}, h^{\prime}}\left(\omega^{*}\right)}\left(J^{*}\right)-O_{\mathbf{p}}(\epsilon)-o_{\epsilon, \mathbf{p}}^{k-h \rightarrow \infty}(1),
\end{aligned}
$$

Here $J^{*}$ denotes, provided that $J$ is a subinterval of $\left[k \chi, k \chi+D^{\prime}\right]$, the interval

$$
\left.\left.J^{*}=\left(J-\left(k-k^{*}-\frac{C \epsilon^{2}}{\chi}\right)\right) \cap\left(J-\left(k-k^{*}\right)\right)\right\}\right) \subseteq\left[k^{*} \chi, k^{*} \chi+D^{\prime}\right] .
$$

Since $\left|\left(J-\left(k-k^{*}\right)\right) \backslash J^{*}\right| \leq \frac{C \epsilon^{2}}{\chi}$,

$$
\Gamma_{\mathcal{A}_{k^{*}}^{h^{*}, h^{\prime}}\left(\omega^{*}\right)}\left(J^{*}\right) \geq \Gamma_{\mathcal{A}_{k^{*}}^{h^{*}, h^{\prime}}\left(\omega^{*}\right)}\left(J-\left(k-k^{*}\right)\right)-O_{\mathbf{p}}\left(\epsilon^{2}\right)
$$

by the uniform absolute continuity of the probability distribution $\Gamma_{\mathcal{A}_{k^{*}}^{h^{*} h^{\prime}\left(\omega^{*}\right)}}$ (Lemma 3.5).

Finally, since $\sigma^{\tau_{k^{*}}\left(\omega^{*}\right)} \omega^{*}=\sigma^{\tau_{k}(\omega)} \omega$ (i.e. the $h^{\prime}$ components are the same $\eta^{\prime}$ ), one can check by construction that $\Gamma_{\mathcal{A}_{k^{*}}^{h^{*}, h^{\prime}}\left(\omega^{*}\right)}=U_{-\left(k-k^{*}\right) \chi} \Gamma_{\mathcal{A}_{k}^{h, h^{\prime}}(\omega)}$, where for $x \in \mathbb{R}, U_{x}: \mathbb{R} \rightarrow \mathbb{R}$ is the translation by $x$. So

$$
\Gamma_{\mathcal{A}_{k^{*}}^{h^{*}, h^{\prime}}\left(\omega^{*}\right)}\left(J^{*}\right) \geq \Gamma_{\mathcal{A}_{k}^{k, h^{\prime}}(\omega)}(J)-O_{\mathbf{p}}\left(\epsilon^{2}\right) .
$$
(iii),

To obtain (25), plug (29) into (28), then multiply by (27). This completes the proof of Property Theorem 3.8 is established. 


\section{Proof of Theorem 1.1 part (1)}

In this Section we prove Theorem 1.1part (1). We require a preliminary step, which is an adaptation of Theorem 3.7 for Fourier modes. This is the content of the next subsection:

\subsection{Application of Theorem 3.7 to Fourier modes}

Fix a Borel probability measure $\rho \in \mathcal{P}(\mathbb{R})$. For every $q \in \mathbb{R}$ we define a function $g_{q, \rho}: \mathbb{R} \rightarrow \mathbb{R}$ via

$$
g_{q, \rho}(t)=\mid \mathcal{F}_{q}\left(M_{\left.e^{-t} \rho\right)\left.\right|^{2}}\right.
$$

where we recall that $M_{s}(x)=s \cdot x$ for any $s, x \in \mathbb{R}$.

Next, fixing $h_{0}=0$ in Theorem 3.7 and assuming $k \in \mathbb{N}$, we define the sequence $o_{k}:=o_{0, \mathbf{p}}^{k \rightarrow \infty}$. Notice that the assumption $h_{0}=0$ means that $A_{\eta}$ is the entire symbolic space $\mathcal{A}^{\mathbb{N}}$ (since the only word of length 0 is the empty word). The following Theorem is needed in conjunction with Theorem 3.7. since in practice we will need a version of Theorem 3.7 for functions rather than intervals.

Theorem 4.1. Let $q$ be large, let $C>1$, and let $k=k(q)$ be defined implicitly as an integer satisfying

$$
|q|=\Theta_{C}\left(o_{k}^{-\frac{1}{4}} e^{\left(k+h^{\prime}\right) \chi}\right)
$$

where $h^{\prime}=\sqrt{k}$. Let $\rho \in \mathcal{P}(\mathbb{R})$ be a measure such that

$$
\operatorname{diam}(\operatorname{supp}(\rho))=O\left(e^{-h^{\prime} \chi}\right)
$$

Then for every $\xi \in \widetilde{A}_{k, \eta}^{h, h^{\prime}} \subseteq \mathcal{A}^{\mathbb{N}}$ as in Theorem 3.7, recalling that here $h=h_{0}=0$, we have

$$
\left|\mathbb{E}_{\mathcal{A}_{k}^{h, h^{\prime}}(\xi)}\left[g_{q, \rho}\left(S_{\tau_{k}(\omega)}\right)\right]-\int_{k \chi}^{k \chi+D^{\prime}} g_{q, \rho}(x) d \Gamma_{\mathcal{A}_{k}^{h, h^{\prime}}(\xi)}(x)\right| \leq O\left(o_{k}^{\frac{1}{4}}\right) .
$$

Proof. We first claim that the function $g_{q, \rho}(t)$ is $4 \pi q e^{-\chi k} \cdot \operatorname{diam}(\operatorname{supp}(\rho))$ Lipschitz, whenever $t \in$ $\left[k \chi, k \chi+D^{\prime}\right]$. Indeed, since the complex exponential is a 1-Lipschitz function, for any $x, y \in \operatorname{supp}(\rho)$ and $t, s \in\left[k \chi, k \chi+D^{\prime}\right]$ we have

$\left|\exp \left(2 \pi i q e^{-t}(x-y)\right)-\exp \left(2 \pi i q e^{-s}(x-y)\right)\right| \leq|2 \pi q(x-y)| \cdot\left|e^{-t}-e^{-s}\right| \leq 4 \pi q \operatorname{diam}(\operatorname{supp}(\rho)) \cdot e^{-k \chi}$.

Since the $L^{1}$ norm is always bounded by the $L^{\infty}$ norm, and since

$$
g_{q, \rho}(t)=\left|\mathcal{F}_{q}\left(M_{e^{-t}} \rho\right)\right|^{2}=\iint \exp \left(2 \pi i q e^{-t}(x-y)\right) d \rho(x) d \rho(y)
$$

the Claim follows.

Recalling that $k=k(q)$ satisfies

$$
q=\Theta_{C}\left(o_{k}^{-\frac{1}{4}} e^{\left(k+h^{\prime}\right) \chi}\right)
$$

and that

$$
\operatorname{diam}(\operatorname{supp}(\rho))=O\left(e^{-h^{\prime} \chi}\right)
$$

it follows that the function

$$
t \in\left[k \chi, k \chi+D^{\prime}\right] \mapsto g_{q, \rho}(t)
$$

is $o_{k}^{-\frac{1}{4}}$ Lipschitz (up to a constant universal multiplicative factor). Therefore, there exists a step function $\psi:\left[k \chi, k \chi+D^{\prime}\right] \rightarrow \mathbb{R}$ such that: 
1. $\psi$ consists of $o_{k}^{-\frac{2}{4}}$ steps (indicators of intervals).

2. $\left\|\psi-g_{q, \rho}\right\|_{\infty} \leq o_{k}^{\frac{1}{4}}$ on the interval $\left[k \chi, k \chi+D^{\prime}\right]$.

Thus,

$$
\begin{gathered}
\left|\mathbb{E}_{\mathcal{A}_{k}^{h, h^{\prime}(\xi)}}\left[g_{q, \rho}\left(S_{\tau_{k}(\omega)}\right)\right]-\int_{k \chi}^{k \chi+D^{\prime}} g_{q, \rho}(x) d \Gamma_{\mathcal{A}_{k}^{h, h^{\prime}}(\xi)}(x)\right| \\
\leq\left|\mathbb{E}_{\mathcal{A}_{k}^{h, h^{\prime}}(\xi)}\left[g_{q, \rho}\left(S_{\tau_{k}(\omega)}\right)\right]-\mathbb{E}_{\mathcal{A}_{k}^{h, h^{\prime}}(\xi)}\left[\psi\left(S_{\tau_{k}(\omega)}\right)\right]\right| \\
+\left|\mathbb{E}_{\mathcal{A}_{k}^{h, h^{\prime}(\xi)}}\left[\psi\left(S_{\tau_{k}(\omega)}\right)\right]-\int_{k \chi}^{k \chi+D^{\prime}} \psi(x) d \Gamma_{\mathcal{A}_{k}^{h, h^{\prime}}(\xi)}(x)\right| \\
+\left|\int_{k \chi}^{k \chi+D^{\prime}} \psi(x) d \Gamma_{\mathcal{A}_{k}^{h, h^{\prime}(\xi)}}(x)-\int_{k \chi}^{k \chi+D^{\prime}} g_{q, \rho}(x) d \Gamma_{\mathcal{A}_{k}^{h, h^{\prime}}(\xi)}(x)\right| .
\end{gathered}
$$

Now, the terms in (31) and (33) are bounded by $o_{k}^{\frac{1}{4}}$ by point 2 above.

Finally, the term in (32) is bounded by $o_{k}^{-\frac{2}{4}} \cdot o_{k}$ since by point 1 above there are at most $o_{k}^{-\frac{2}{4}}$ steps in $\psi$, and since by Theorem 3.7 each such step introduces an error of at most $o_{k}$.

In the context of Theorem 4.1, it is natural to ask about the existence of integers $k$ that satisfy (30) with respect to some uniform $C>1$. This is the content of the following Lemma:

Lemma 4.2. By potentially making ok go to zero slower, we may assume that there exists a constant $C>1$ such that for every $q$ large there exists $k=k(q) \in \mathbb{N}$ such that

$$
|q|=\Theta_{C}\left(o_{k}^{-\frac{1}{4}} e^{(k+\sqrt{k}) \chi}\right) .
$$

Proof. We first make the following assumptions on the sequence $o_{k}$ :

1. $o_{k} \geq \frac{1}{k}$. Otherwise, we move to the sequence $a_{k}=\max \left\{o_{k}, \frac{1}{k}\right\}$. Then $o_{k} \leq a_{k}$ and still $a_{k} \rightarrow 0$.

2. It is monotonic decreasing. Otherwise, for every $k$ define $v_{k}=\sup \left\{o_{n}: n \geq k\right\}$. Then $o_{k} \leq v_{k}$, $v_{k}$ is decreasing, and it is clear that $v_{k} \rightarrow 0$.

3. For every $k$ we have $\frac{1}{4} \cdot o_{k} \leq o_{k+1} \leq o_{k}$. Otherwise, we move to the recursively defined sequence $b_{k}$, where

$$
b_{1}=o_{1}, \quad b_{k}=\max \left\{o_{k}, \frac{b_{k-1}}{4}\right\} .
$$

Then $b_{k} \geq o_{k} \geq \frac{1}{k}$ and

$$
\frac{b_{k}}{4} \leq b_{k+1} \leq b_{k}, \quad b_{k} \rightarrow 0 .
$$

Now, let $g: \mathbb{R}_{+} \rightarrow \mathbb{R}_{+}$be a smooth monotonic decreasing function such that $g(k)=o_{k}$. Let $q$ be large. Find $x \in \mathbb{R}_{+}$such that

$$
|q|=g(x)^{-\frac{1}{4}} \cdot e^{(x+\sqrt{x}) \chi} .
$$

Notice that

$$
\left|\frac{1}{4} \log \frac{1}{g([x])}+(x+\sqrt{x}) \chi-\frac{1}{4} \log \frac{1}{g(x)}-([x]+\sqrt{[x]}) \chi\right|
$$




$$
\begin{gathered}
\leq\left|\frac{1}{4} \log \frac{g(x)}{g([x])}\right|+|(x+\sqrt{x}-[x]-\sqrt{[x]}) \cdot \chi| \\
\leq \frac{1}{4}\left|\log \frac{g([x]+1)}{g([x])}\right|+3 \chi \\
\leq \frac{1}{4}|\log 4|+3 \chi .
\end{gathered}
$$

It follows that

$$
\frac{g(x)^{-\frac{1}{4}} \cdot e^{(x+\sqrt{x}) \chi}}{g([x])^{-\frac{1}{4}} \cdot e^{([x]+\sqrt{[x]} \chi}}=\exp \left(\frac{1}{4} \log \frac{1}{g([x])}+(x+\sqrt{x}) \chi-\frac{1}{4} \log \frac{1}{g(x)}-([x]+\sqrt{[x]}) \chi\right)=O(1) .
$$

We thus choose, for every $q$ large, our $k$ as $[x]$. It follows that there is some uniform $C>1$ such that

$$
C^{-1} \leq \frac{|q|}{o_{k}^{-\frac{1}{4}} e^{(k+\sqrt{k}) \chi}} \leq C
$$

which is what we claimed.

\subsection{Proof of Theorem 1.1 part (1)}

Let $\nu$ be as in Theorem 1.1. Our goal is to show that

$$
\lim _{|q| \rightarrow \infty} \mathcal{F}_{q}(\nu)=0 .
$$

So, let $\epsilon>0$, let $|q|$ be large, and choose $k=k(q) \in \mathbb{N}$ as in Lemma 4.2, Recall that this means that for some $C>1$,

$$
q=\Theta_{C}\left(o_{k}^{-\frac{1}{4}} \cdot e^{\left(k+h^{\prime}\right) \chi}\right)
$$

where our standing assumption is that

$$
h^{\prime}=\sqrt{k} .
$$

By Lemma 4.2 (and its proof), any requirement that $k$ be large translates to a requirement on $q$ being large. In the notation of Theorem 3.7, we let $k$ be large, and fix $h_{0}=0$. Recall that $h_{0}=0$ means that $h=0$ and so $A_{\eta}=\mathcal{A}^{\mathbb{N}}$. We also define an auxiliary stopping time $\tilde{\beta}_{k}: \mathcal{A}^{\mathbb{N}} \rightarrow \mathbb{N}$ by

$$
\tilde{\beta}_{k}(\omega)=\min \left\{m:\left|f_{\left.\omega\right|_{m}}^{\prime}\left(x_{0}\right)\right|<e^{-\left(k+h^{\prime}\right) \chi}\right\}
$$

where we recall that $x_{0} \in I$ is our prefixed point as in Section 1.2.1.

Lemma 4.3. For every $k \in \mathbb{N}$,

$$
\nu=\mathbb{E}\left(f_{\left.\omega\right|_{\tilde{\beta}_{k}(\omega)}} \nu\right) .
$$

Proof. This is standard, and follows since $\nu$ is self-conformal. See e.g. [4, Lemma 2.2.4].

So, by Lemma 4.3 and Jensen's inequality we obtain

$$
\begin{aligned}
\left|\mathcal{F}_{q}(\nu)\right|^{2} & =\left|\mathcal{F}_{q}\left(\mathbb{E}\left(f_{\left.\omega\right|_{\tilde{\beta}_{k}(\omega)}} \nu\right)\right)\right|^{2} \\
& =\left|\mathbb{E}\left(\mathcal{F}_{q}\left(f_{\left.\omega\right|_{\tilde{\beta}_{k}(\omega)}} \nu\right)\right)\right|^{2} \\
& \leq \mathbb{E}\left(\left|\mathcal{F}_{q}\left(f_{\left.\omega\right|_{\tilde{\beta}_{k}(\omega)}} \nu\right)\right|^{2}\right) .
\end{aligned}
$$


Next, appealing to Theorem [3.7 with our choice of $k, h_{0}, h^{\prime}$, there is a subset that we denote by $\tilde{A_{\eta}} \subseteq \mathcal{A}^{\mathbb{N}}$ with $\mathbb{P}\left(\tilde{A_{\eta}}\right) \geq 1-o_{k}(1)$ such that

$$
\begin{gathered}
\mathbb{E}\left(\left|\mathcal{F}_{q}\left(f_{\left.\omega\right|_{\tilde{\beta}_{k}(\omega)}} \nu\right)\right|^{2}\right)= \\
=\int_{\xi \in \mathcal{A}^{\mathbb{N}} \backslash \tilde{A}_{\eta}} \mathbb{E}_{\mathcal{A}_{k}^{h, h^{\prime}(\xi)}}\left|\mathcal{F}_{q}\left(f_{\left.\omega\right|_{\tilde{\beta}_{k}(\omega)}} \nu\right)\right|^{2} d \mathbb{P}(\xi)+\int_{\xi \in \tilde{A}_{\eta}} \mathbb{E}_{\mathcal{A}_{k}^{h, h^{\prime}(\xi)}}\left|\mathcal{F}_{q}\left(f_{\left.\omega\right|_{\tilde{\beta}_{k}(\omega)}} \nu\right)\right|^{2} d \mathbb{P}(\xi) .
\end{gathered}
$$

Combining this with the previous equation array, and using that $\left|\mathcal{F}_{q}\left(f_{\left.\omega\right|_{\tilde{\beta}_{k}(\omega)}} \nu\right)\right|^{2} \leq 1$ uniformly in all parameters, we conclude that

$$
\left|\mathcal{F}_{q}(\nu)\right|^{2} \leq \int_{\xi \in \tilde{A_{\eta}}} \mathbb{E}_{\mathcal{A}_{k}^{h, h^{\prime}}(\xi)}\left|\mathcal{F}_{q}\left(f_{\left.\omega\right|_{\tilde{\beta}_{k}(\omega)}} \nu\right)\right|^{2} d \mathbb{P}(\xi)+o_{k}(1)
$$

Next, we take a closer look at the maps $f_{\left.\omega\right|_{\tilde{\beta}_{k}(\omega)}}$ :

Lemma 4.4. There exists some integer $P>1$ such that:

For all $k$ large enough, and for every $\omega$, letting $\eta^{\prime}$ be such that $A_{k, \eta, \eta^{\prime}}=\mathcal{A}_{k}^{h, h^{\prime}}(\omega)$, we have

$$
\left|\tilde{\beta}_{k}(\omega)-\tau_{k}(\omega)-\right| \eta^{\prime}|| \leq P .
$$

Proof. We first observe that, by the definition of $\tilde{\beta}_{k}$ from (34),

$$
f_{\left.\omega\right|_{\tilde{\beta}_{k}(\omega)}}=f_{\left.\omega\right|_{\tau_{k}(\omega)}} \circ f_{\left.\omega\right|_{\tau_{k}(\omega)} ^{\tilde{\beta}_{k}(\omega)}}, \quad \text { and } f_{\left.\omega\right|_{\tau_{k}(\omega)+\left|\eta^{\prime}\right|}}=f_{\left.\omega\right|_{\tau_{k}(\omega)}} \circ f_{\eta^{\prime}} \text { since } \omega \in A_{k, \eta, \eta^{\prime}}
$$

So, either $\left.\omega\right|_{\tau_{k}(\omega)} ^{\tilde{\beta}_{k}(\omega)}$ is a prefix of $\eta^{\prime}$, or vice versa. By the last displayed equation, for any $x \in I$,

$$
\frac{\left|f_{\left.\omega\right|_{\tilde{\beta}_{k}(\omega)}}^{\prime}(x)\right|}{\left|f_{\left.\omega\right|_{\tau_{k}(\omega)}}^{\prime}\left(f_{\left.\omega\right|_{\tau_{k}(\omega)} ^{\tilde{\beta}_{k}(\omega)}}(x)\right)\right|}=\left|f_{\left.\omega\right|_{\tau_{k}(\omega)} ^{\tilde{\beta}_{k}(\omega)}}^{\prime}(x)\right| .
$$

Now, it is a consequence of Theorem 2.1 (bounded distortion), the definition of $\tilde{\beta}_{k}$, and of the definition of $\tau_{k}$ (Section [3), that for some $L>1$ and all $y \in I$,

$$
L^{-1} \cdot e^{-\left(k+h^{\prime}\right) \chi-D^{\prime}} \leq\left|f_{\omega \tilde{\beta}_{k}(\omega)}^{\prime}(y)\right| \leq L \cdot e^{-\left(k+h^{\prime}\right) \chi}, \quad L^{-1} \cdot e^{-k \chi-D^{\prime}} \leq\left|f_{\left.\omega\right|_{\tau_{k}}(\omega)}^{\prime}(y)\right| \leq L \cdot e^{-k \chi}
$$

combining the last two displayed equations, we see that there some constant $C^{\prime}>1$ such that

$$
\left|f_{\left.\omega\right|_{\tau_{k}(\omega)} ^{\tilde{\beta}_{k}(\omega)}}^{\prime}(x)\right|=\Theta_{C^{\prime}}\left(e^{-h^{\prime} \chi}\right), \quad \forall x \in I .
$$

On the other hand, by the definition of the event $A_{k, \eta, \eta^{\prime}}$ and by Theorem 2.1

$$
\left|f_{\eta^{\prime}}(x)\right|=\Theta_{L}\left(e^{-h^{\prime} \chi}\right), \quad \forall x \in I
$$

Therefore, combining equation (38) with (37) (and noting that the constants $C^{\prime}, L$ are uniform), that either $\left.\omega\right|_{\tau_{k}(\omega)} ^{\tilde{\beta}_{k}(\omega)}$ is a prefix of $\eta^{\prime}$ or vice versa, and equation (44), the Lemma follows. 
Let $P$ be as in Lemma 4.4, For every word $\eta^{\prime} \in\{1, \ldots, n\}^{*}$ of length $\left|\eta^{\prime}\right|>P$ we define

$$
\overline{\eta^{\prime}}:=\left.\eta^{\prime}\right|_{\left|\eta^{\prime}\right|-P}
$$

That is, $\overline{\eta^{\prime}}$ is the prefix of $\eta^{\prime}$ of length $\left|\eta^{\prime}\right|-P$. It is now a corollary of Lemma 4.4 that for any $\omega$, if $\eta^{\prime}=\eta^{\prime}(\omega)$ is as in Lemma 4.4, then there is a word $\rho_{\omega, k}$ such that

$$
f_{\left.\omega\right|_{\tilde{\beta}_{k}(\omega)}}=f_{\left.\omega\right|_{\tau_{k}(\omega)}} \circ f_{\overline{\eta^{\prime}}} \circ f_{\rho_{\omega, k}}
$$

and $\left|\rho_{\omega, k}\right| \leq 2 P$.

With this information, we revisit equation (35). Recall that $M_{s}(t)=s \cdot t$.

Claim 4.5. Fix $\beta \in(0, \gamma)$. Then for all $k$ large enough,

$$
\begin{aligned}
& \left|\mathcal{F}_{q}(\nu)\right|^{2} \leq \int_{\xi \in \tilde{A_{\eta}}} \mathbb{E}_{\mathcal{A}_{k}^{h, h^{\prime}(\xi)}}\left|\mathcal{F}_{q}\left(M_{\left.e^{-S_{\tau_{k}(\omega)}(\omega)} \circ M_{\operatorname{sign}\left(f_{\omega \mid}^{\prime} \tau_{k}(x)\right.}\left(x_{\sigma^{\tau_{k}(\omega)}(\omega)}\right)\right)} \circ f_{\overline{\eta^{\prime}}} \circ f_{\rho_{\omega, k}} \nu\right)\right|^{2} d \mathbb{P}(\xi) \\
& +O\left(q \cdot e^{-\left(k+h^{\prime}\right) \chi-\beta \cdot h^{\prime} \chi}\right)+o_{k}(1)
\end{aligned}
$$

where for every $\xi \in \tilde{A}_{\eta}$, recalling that $\mathcal{A}_{k}^{h, h^{\prime}}(\xi)=A_{k, \eta, \eta^{\prime}}, \eta^{\prime}$ is defined as $\eta^{\prime}=\eta^{\prime}(\xi)$.

Proof. Fix $\xi \in \tilde{A}_{\eta}$. Assuming $\mathcal{A}_{k}^{h, h^{\prime}}(\xi)=A_{k, \eta, \eta^{\prime}}$, let $\eta^{\prime}$ be this $\eta^{\prime}=\eta^{\prime}(\xi)$. Assume $\omega \in A_{k, \eta, \eta^{\prime}}$. Then we have seen that there is a word $\rho_{\omega, k}$ such that

$$
f_{\left.\omega\right|_{\tilde{\beta}_{k}(\omega)}}=f_{\left.\omega\right|_{\tau_{k}(\omega)}} \circ f_{\overline{\eta^{\prime}}} \circ f_{\rho_{\omega, k}}
$$

and $\left|\rho_{\omega, k}\right| \leq 2 P$. It follows from the proof of Lemma 4.4 (specifically, equation (37)) that there some constant $C^{\prime}>1$ such that

$$
\left|\left(f_{\overline{\eta^{\prime}}} \circ f_{\rho_{\omega, k}}\right)^{\prime}(x)\right|=\Theta_{C^{\prime}}\left(e^{-h^{\prime} \chi}\right), \quad \forall x \in I .
$$

Also, it is a consequence of (39) that there exists some $z \in I$ with $f_{\overline{\eta^{\prime}}} \circ f_{\rho_{\omega, k}}(z)=x_{\sigma^{\tau} k(\omega)}(\omega)$.

Now, plug into the $C^{0}$ linearization Lemma 2.3 the parameters $g=f_{\left.\omega\right|_{\tau_{k}(\omega)}}, y=f_{\bar{\eta}^{\prime}} \circ f_{\rho_{\omega, k}}(z)$ and for $x \in I$ we plug in $f_{\bar{\eta}^{\prime}} \circ f_{\rho_{\omega, k}}(x)$. Then, by (39) and assuming $k$ (and therefore $h^{\prime}$ ) are large enough,

$$
\begin{gathered}
\left|f_{\left.\omega\right|_{\tilde{\beta}_{k}(\omega)}}(x)-f_{\left.\omega\right|_{\tau_{k}(\omega)}}\left(f_{\overline{\eta^{\prime}}} \circ f_{\rho_{\omega, k}}(z)\right)-f_{\left.\omega\right|_{\tau_{k}(\omega)}}^{\prime}\left(f_{\overline{\eta^{\prime}}} \circ f_{\rho_{\omega, k}}(z)\right)\left(f_{\overline{\eta^{\prime}}} \circ f_{\rho_{\omega, k}}(x)-f_{\overline{\eta^{\prime}}} \circ f_{\rho_{\omega, k}}(z)\right)\right| \\
\leq\left|f_{\left.\omega\right|_{\tau_{k}(\omega)}}^{\prime}\left(f_{\overline{\eta^{\prime}}} \circ f_{\rho_{\omega, k}}(z)\right)\right| \cdot\left|f_{\overline{\eta^{\prime}}} \circ f_{\rho_{\omega, k}}(x)-f_{\overline{\eta^{\prime}}} \circ f_{\rho_{\omega, k}}(z)\right|^{1+\beta} .
\end{gathered}
$$

And, by the definition of $\tau_{k}$, since $f_{\overline{\eta^{\prime}}} \circ f_{\rho_{\omega, k}}(z)=x_{\sigma^{\tau} k(\omega)(\omega)}$, and by (40)

$$
\left|f_{\left.\omega\right|_{\tau_{k}(\omega)}}^{\prime}\left(f_{\overline{\eta^{\prime}}} \circ f_{\rho_{\omega, k}}(z)\right)\right| \leq e^{-k \chi}, \quad\left|f_{\bar{\eta}^{\prime}} \circ f_{\rho_{\omega, k}}(x)-f_{\overline{\eta^{\prime}}} \circ f_{\rho_{\omega, k}}(z)\right| \leq O\left(e^{-h^{\prime} \chi}\right) .
$$

Now, for every $\omega \in A_{k, \eta, \eta^{\prime}}$ we define a smooth map $S_{\omega, k, \eta^{\prime}}: I \rightarrow \mathbb{R}$ via

$$
\begin{aligned}
S_{\omega, k, \eta^{\prime}}(x)= & \left|f_{\left.\omega\right|_{\tau_{k}(\omega)}}^{\prime}\left(x_{\sigma^{\tau_{k}(\omega)}(\omega)}\right)\right| \cdot \operatorname{sign}\left(f_{\left.\omega\right|_{\tau_{k}(\omega)}}^{\prime}\left(x_{\sigma^{\tau_{k}(\omega)}(\omega)}\right)\right) \cdot f_{\bar{\eta}^{\prime}} \circ f_{\rho_{\omega, k}}(x) \\
& -f_{\left.\omega\right|_{\tau_{k}(\omega)}}^{\prime}\left(x_{\sigma^{\tau_{k}(\omega)}(\omega)}\right) \cdot x_{\sigma^{\tau_{k}(\omega)}(\omega)}+f_{\left.\omega\right|_{\tau_{k}(\omega)}}^{\prime}\left(x_{\sigma^{\tau_{k}(\omega)}(\omega)}\right) .
\end{aligned}
$$


This map is affine in $\operatorname{sign}\left(f_{\left.\omega\right|_{\tau_{k}(\omega)}}^{\prime}\left(x_{\sigma^{\tau} k^{(\omega)}(\omega)}\right)\right) \cdot f_{\overline{\eta^{\prime}}} \circ f_{\rho_{\omega, k}}(x)$. Then we have just shown that

$$
\left\|f_{\left.\omega\right|_{\tilde{\beta}_{k}(\omega)}}-S_{\omega, k, \eta^{\prime}}\right\|_{C^{0}(I)} \leq O\left(e^{-\left(k+h^{\prime}\right) \chi-\beta h^{\prime} \chi}\right) .
$$

So, since $\mathcal{F}_{q}(\cdot)$ is a $2 \pi q$-Lipschitz function,

$$
\left|\mathcal{F}_{q}\left(f_{\left.\omega\right|_{\tilde{\beta}_{k}(\omega)}} \nu\right)-\mathcal{F}_{q}\left(S_{\omega, k, \eta^{\prime}} \nu\right)\right| \leq O\left(q \cdot e^{-\left(k+k^{\prime}\right) \chi-\beta k^{\prime} \chi}\right) .
$$

Therefore

$$
\left.|| \mathcal{F}_{q}\left(f_{\left.\omega\right|_{\tilde{\beta}_{k}(\omega)}} \nu\right)\right|^{2}-\left|\mathcal{F}_{q}\left(S_{\omega, k, \eta^{\prime}} \nu\right)\right|^{2} \mid \leq O\left(q \cdot e^{-\left(k+h^{\prime}\right) \chi-\beta h^{\prime} \chi}\right) .
$$

and so for every event $A_{k, \eta, \eta^{\prime}}$ we have

$$
\left.\left|\mathbb{E}_{A_{k, \eta, \eta^{\prime}}}\right| \mathcal{F}_{q}\left(f_{\left.\omega\right|_{\tilde{\beta}_{k}(\omega)}} \nu\right)\right|^{2}-\mathbb{E}_{A_{k, \eta, \eta^{\prime}}}\left|\mathcal{F}_{q}\left(S_{\omega, k, \eta^{\prime}} \nu\right)\right|^{2} \mid \leq O\left(q \cdot e^{-\left(k+h^{\prime}\right) \chi-\beta h^{\prime} \chi}\right) .
$$

Finally, recall equation (35), and recall the definition of the maps $S_{\omega, k, \eta^{\prime}}$ from (41). Note that

$$
\log \left|f_{\left.\omega\right|_{\tau_{k}(\omega)}}^{\prime}\left(x_{\sigma^{\tau_{k}(\omega)}(\omega)}\right)\right|=-S_{\tau_{k}(\omega)}(\omega)
$$

by Lemma 3.2. The Claim follows from (35) and (42), since the translation of $S_{\omega, k, \eta^{\prime}}$ does not effect the absolute value of $\mathcal{F}_{q}(\cdot)$, by integrating over all $\xi \in \tilde{A}_{\eta}$ (using that the bounds we got are uniform in $\xi$ ).

Corollary 4.6. There is some $K_{1}=K_{1}(\epsilon)$ such that for all $k>K_{1}$,

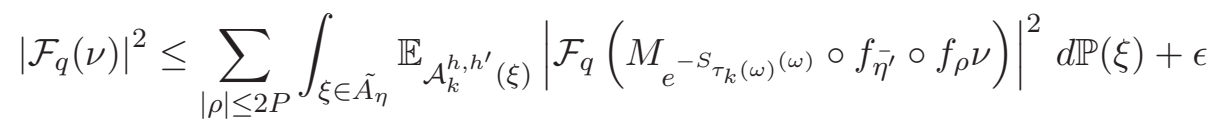

where $P$ is the constant from Lemma 4.4. Furthermore, there is some global constant $C^{\prime}>1$ such that for all $\overline{\eta^{\prime}}$ and $\rho$ as above

$$
\left|\left(f_{\overline{\eta^{\prime}}} \circ f_{\rho}\right)^{\prime}(x)\right|=\Theta_{C^{\prime}}\left(e^{-h^{\prime} \chi}\right), \quad \forall x \in I .
$$

Remark 4.7. For notational convenience, in this Corollary and the subsequent argument, we make the assumption that we always have $f_{\left.\omega\right|_{\tau_{k}(x)}}^{\prime}\left(x_{\sigma^{\tau_{k}(\omega)}(\omega)}\right)>0$. Otherwise, we simply make the sum on the right hand side of (43) larger, by including the possibility that it is negative. Since there are uniformly finitely many such options, still the sum above is over uniformly finitely many terms, and the proof follows through.

Proof. For every $k$ large enough, for every $\xi \in \tilde{A}_{\eta}$ and every $\omega \in \mathcal{A}_{k}^{h, h^{\prime}}(\xi)$, as $\left|\rho_{\omega, k}\right| \leq 2 P$

$$
\left|\mathcal{F}_{q}\left(M_{e^{-S_{\tau_{k}}(\omega)}(\omega)} \circ f_{\overline{\eta^{\prime}}} \circ f_{\rho_{\omega, k}} \nu\right)\right|^{2} \leq \sum_{|\rho| \leq 2 P}\left|\mathcal{F}_{q}\left(M_{e^{-S_{\tau_{k}}(\omega)}}(\omega) \circ f_{\overline{\eta^{\prime}}} \circ f_{\rho} \nu\right)\right|^{2}
$$

and so, by Claim 4.5, assuming $f_{\left.\omega\right|_{\tau_{k}(x)}}^{\prime}\left(x_{\sigma^{\tau_{k}(\omega)}(\omega)}\right)>0$ is always true,

$$
\left|\mathcal{F}_{q}(\nu)\right|^{2} \leq \int_{\xi \in \tilde{A_{\eta}}} \mathbb{E}_{\mathcal{A}_{k}^{h, h^{\prime}}(\xi)}\left|\mathcal{F}_{q}\left(M_{e^{-S_{\tau_{k}}(\omega)}(\omega)} \circ f_{\bar{\eta}^{\prime}} \circ f_{\rho_{\omega, k}} \nu\right)\right|^{2} d \mathbb{P}(\xi)+O\left(q \cdot e^{-\left(k+h^{\prime}\right) \chi-\beta \cdot h^{\prime} \chi}\right)+o_{k}(1)
$$




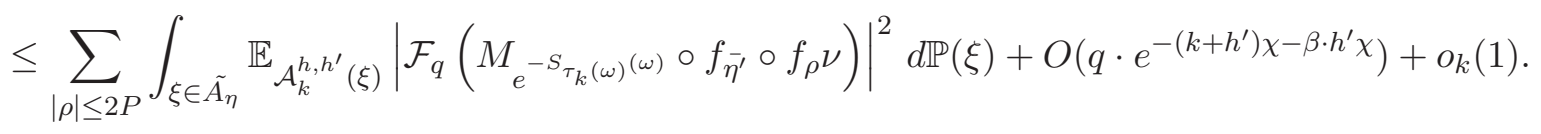

Recalling the choice of $k=k(q)$ (which is as in Lemma 4.2),

$$
O\left(q \cdot e^{-\left(k+h^{\prime}\right) \chi-\beta h^{\prime} \chi}\right)=O\left(o_{k}^{-\frac{1}{4}} \cdot e^{-\beta h^{\prime} \chi}\right) .
$$

So, since $o_{k}$ decays in at most a polynomial rate (by e.g. Lemma 4.2), there is some $K_{1}=K_{1}(\epsilon)$ as we claimed. The last assertion is a consequence of equation (38), Theorem 2.1 (bounded distortion), that $|\rho| \leq 2 P$, and of equation (4).

Now, fix some $\rho$ with $|\rho| \leq 2 P$ and consider the corresponding term in (43)

$$
\int_{\xi \in \tilde{A}_{\eta}} \mathbb{E}_{\mathcal{A}_{k}^{h, h^{\prime}}(\xi)}\left|\mathcal{F}_{q}\left(M_{e^{-S_{\tau_{k}}(\omega)}}(\omega) \circ f_{\bar{\eta}^{\prime}} \circ f_{\rho} \nu\right)\right|^{2} d \mathbb{P}(\xi) .
$$

We next appeal to Theorem 4.1 for every event $\mathcal{A}_{k}^{h, h^{\prime}}(\xi)$ separately. To do this, we notice that by Corollary 4.6, for every $f_{\overline{\eta^{\prime}}} \circ f_{\rho}$ involved

$$
\operatorname{diam}\left(\operatorname{supp}\left(f_{\overline{\eta^{\prime}}} \circ f_{\rho} \nu\right)\right)=O\left(e^{-h^{\prime} \chi}\right) .
$$

Notice that the the error term in Theorem 4.1 is $O\left(o_{k}^{\frac{1}{4}}\right)$ independently of the event $\mathcal{A}_{k}^{h, h^{\prime}}(\xi)$. So,

$$
\begin{gathered}
\int_{\xi \in \tilde{A_{\eta}}} \mathbb{E}_{\mathcal{A}_{k}^{h, h^{\prime}}(\xi)}\left|\mathcal{F}_{q}\left(M_{e^{-S_{\tau_{k}}(\omega)}}(\omega) \circ f_{\overline{\eta^{\prime}}} \circ f_{\rho} \nu\right)\right|^{2} d \mathbb{P}(\xi) \\
\leq \int_{\xi \in \tilde{A_{\eta}}} \int_{k \chi}^{k \chi+D^{\prime}}\left|\mathcal{F}_{q}\left(M_{e^{-x}} \circ f_{\overline{\eta^{\prime}}} \circ f_{\rho} \nu\right)\right|^{2} d \Gamma_{\mathcal{A}_{k}^{h, h^{\prime}}(\xi)}(x) d \mathbb{P}(\xi)+O\left(o_{k}^{\frac{1}{4}}\right) .
\end{gathered}
$$

Let $K_{2}=K_{2}(\epsilon)$ be large enough to ensure that if $k \geq K_{2}$ then $O\left(o_{k}^{\frac{1}{4}}\right) \leq \frac{\epsilon}{\{\rho:|\rho| \leq 2 P\} \mid}$. Then, since this is true for every $\rho$ with $|\rho| \leq 2 P$, we see that for $k \geq \max \left\{K_{2}, K_{1}\right\}$, putting this into (43) we get

$$
\left|\mathcal{F}_{q}(\nu)\right|^{2} \leq \sum_{|\rho| \leq 2 P} \int_{\xi \in \tilde{A_{\eta}}} \int_{k \chi}^{k \chi+D^{\prime}}\left|\mathcal{F}_{q}\left(M_{e^{-x}} \circ f_{\overline{\eta^{\prime}}} \circ f_{\rho} \nu\right)\right|^{2} d \Gamma_{\mathcal{A}_{k}^{h, h^{\prime}}(\xi)}(x) d \mathbb{P}(\xi)+2 \epsilon .
$$

Recall that by Lemma 3.5, the probability measure $\Gamma_{\mathcal{A}_{k}^{h, h^{\prime}}(\xi)}$ is absolutely continuous with respect to the Lebesgue measure on $\left[k \chi, k \chi+D^{\prime}\right]$, such that the norm of its density function is uniformly bounded by $\frac{1}{D}>0$ independently of all parameters. Using this fact, as long as $k \geq \max \left\{K_{1}, K_{2}\right\}$

$$
\left|\mathcal{F}_{q}(\nu)\right|^{2} \leq \sum_{|\rho| \leq 2 P} \int_{\xi \in \tilde{A_{\eta}}}\left(\int_{k \chi}^{k \chi+D^{\prime}}\left|\mathcal{F}_{q}\left(M_{e^{-z}} \circ f_{\bar{\eta}^{\prime}} \circ f_{\rho} \nu\right)\right|^{2} \cdot \frac{1}{D} d z\right) d \mathbb{P}(\xi)+2 \epsilon .
$$

We now invoke Lemma 2.6. Taking the measure(s) to be $f_{\bar{\eta}^{\prime}} \circ f_{\rho} \nu$, for any $r>0$, we get the inequality (as long as $k$ is large enough)

$$
\left|\mathcal{F}_{q}(\nu)\right|^{2} \leq \sum_{|\rho| \leq 2 P} \int_{\xi \in \tilde{A_{\eta}}} \frac{D^{\prime}}{D} \cdot\left(\frac{e^{2}}{r \cdot|q|}+\int f_{\overline{\eta^{\prime}}} \circ f_{\rho} \nu\left(B_{e^{\chi^{k} \cdot r}}(y)\right) d\left(f_{\overline{\eta^{\prime}}} \circ f_{\rho} \nu(y)\right)\right) d \mathbb{P}(\xi)+2 \epsilon .
$$


By Corollary 4.6, all the maps $\left(f_{\bar{\eta}^{\prime}} \circ f_{\rho}\right)^{-1}$ as above are $O\left(e^{h^{\prime} \chi}\right)$ Lipschitz (with the implied constant in the $O(\cdot)$ being uniform). Therefore, there is some $T>1$ such that for every $\eta^{\prime}$ and $y \in I$,

$$
\left(f_{\overline{\eta^{\prime}}} \circ f_{\rho}\right)^{-1}\left(B_{e^{\chi k} \cdot r}\left(f_{\overline{\eta^{\prime}}} \circ f_{\rho}(y)\right) \subseteq B_{T \cdot e^{\chi\left(k+h^{\prime}\right) \cdot r}}(y)\right.
$$

so, for a fixed $r>0$ we can relax the dependence on both $\rho$ and $\xi$, and get

$$
\left|\mathcal{F}_{q}(\nu)\right|^{2} \leq|\{\rho:|\rho| \leq 2 P\}| \cdot\left(\frac{e^{2}}{r \cdot|q|}+\int \nu\left(B_{T \cdot e^{\chi\left(k+h^{\prime}\right) \cdot r}}(y)\right) d \nu(y)\right) \cdot \frac{D^{\prime}}{D}+2 \epsilon .
$$

By Lemma 2.2 there exists some $\delta=\delta(\epsilon)>0$ such that

$$
\nu\left(B_{\delta}(y)\right)<\frac{\epsilon \cdot D}{2 \cdot D^{\prime} \cdot|\{\rho:|\rho| \leq 2 P\}|}, \quad \forall y \in \mathbb{R} .
$$

Now, we choose $r$ so that $T \cdot e^{\chi\left(k+k^{\prime}\right)} \cdot r=\delta$. This implies that $\nu\left(B_{T \cdot e^{\chi\left(k+h^{\prime}\right.} \cdot r}(y)\right) \leq \frac{\epsilon \cdot D}{2 \cdot D^{\prime} \cdot|\{\rho:|\rho| \leq 2 P\}|}$ for every $y$. Therefore, $\frac{1}{r}=\frac{T \cdot e^{\chi\left(k+h^{\prime}\right)}}{\delta}$. So, as $|q|=\Theta_{C}\left(o_{k}^{-\frac{1}{4}} \cdot e^{\left(k+h^{\prime}\right) \chi}\right)$,

$$
\frac{e^{2}}{r \cdot|q|}=e^{2} \cdot T \cdot \frac{e^{\chi\left(k+h^{\prime}\right)}}{\delta \cdot|q|} \leq C \cdot e^{2} \cdot T \cdot o_{k}^{\frac{1}{4}} \cdot \frac{e^{\chi\left(k+h^{\prime}\right)}}{\delta \cdot e^{\chi\left(k+h^{\prime}\right)}}=o_{k}^{\frac{1}{4}} \cdot \frac{e^{2} \cdot T \cdot C}{\delta} .
$$

So, as long as $k \geq K_{3}=K_{3}(\epsilon)$,

$$
\frac{e^{2}}{r \cdot|q|} \leq \frac{\epsilon \cdot D}{2 \cdot D^{\prime} \cdot|\{\rho:|\rho| \leq 2 P\}|} .
$$

Finally, if $k \geq \max \left\{K_{1}, K_{2}, K_{3}\right\}$, plugging (45) and (46) into (44),

$$
\left|\mathcal{F}_{q}(\nu)\right|^{2} \leq 3 \epsilon
$$

which implies the Theorem.

\section{Proof of Theorem 1.1 part (2)}

\subsection{Some reductions}

We continue to assume the condition of Theorem 1.1, and use the notation introduced in Sections 1.2.1, 2, 3, Fix an integer $p \geq 2$. We aim to prove the following Theorem:

Theorem 5.1. Let $\nu$ be a measure as in Theorem 1.1. Then for $\nu$ almost every $x$,

$$
\lim _{N} \frac{1}{N} \sum_{n=1}^{N} \delta_{T_{p}^{n}(x)}=\lambda_{[0,1]}
$$

where $\lambda_{[0,1]}$ is the Lebesgue measure on $[0,1]$.

Since $p$ is arbitrary, Theorem 5.1 implies Theorem 1.1 part (2). We will need the following definitions: let $\pi: \mathbb{R} \rightarrow \mathbb{T}=\mathbb{R} / \mathbb{Z} \simeq[0,1)$ be the projection

$$
\pi(x)=x \bmod 1 .
$$


Let $\bar{T}_{p}: \mathbb{T} \rightarrow \mathbb{T}$ be the continuous map

$$
\bar{T}_{p}(y)=p \cdot y \bmod 1 .
$$

Notice that for any $x \in \mathbb{R}$ and any $n \in \mathbb{N}$ we have

$$
\bar{T}_{p}^{n} \circ \pi(x)=\pi \circ T_{p}^{n}(x)=p^{n} \cdot x \bmod 1 .
$$

Our first step is to reduce to the following statement, where we make use of the fact that $\nu$ is the push-forward of $\mathbb{P}$ under $\omega \mapsto x_{\omega}$.

Theorem 5.2. For every $\epsilon>0$ there exists $q^{*}=q^{*}(\epsilon) \in \mathbb{N}$ such that for all integers $q$ with $|q| \geq q^{*}$ and for $\mathbb{P}$ almost every $\omega$,

$$
\underset{N}{\limsup }\left|\mathcal{F}_{q}\left(\frac{1}{N} \sum_{n=1}^{N} \delta_{T_{p}^{n}\left(x_{\omega}\right)}\right)\right|<\epsilon .
$$

Proof that Theorem 5.2 implies Theorem 5.1 Let $\omega$ be a $\mathbb{P}$ typical point, and let $\nu_{\infty}$ be a weak-* limit of the sequence

$$
\frac{1}{N} \sum_{n=1}^{N} \delta_{T_{p}^{n}\left(x_{\omega}\right)}
$$

We will show that $\nu_{\infty}$ is the Lebesgue measure on $[0,1]$. It suffices to show that $\mathcal{F}_{q}\left(\nu_{\infty}\right)=0$ for every integer $q \neq 0$. Consider the measure $\pi \nu_{\infty}$ on $\mathbb{T}$ : It is a consequence of (47) that $\pi \nu_{\infty}$ arises from the $\bar{T}_{p}$ orbit of $\pi\left(x_{\omega}\right)$, and so $\pi \nu_{\infty}$ is $\bar{T}_{p}$ invariant. Now, let $\epsilon>0$. Assuming Theorem 5.2 holds true, let $n \in \mathbb{N}$ be large enough so that $\left|q \cdot p^{n}\right| \geq q^{*}(\epsilon)$. Then

$$
\begin{aligned}
\left|\mathcal{F}_{q}\left(\nu_{\infty}\right)\right| & =\left|\mathcal{F}_{q}\left(\pi \nu_{\infty}\right)\right| \\
& =\left|\mathcal{F}_{q}\left(\bar{T}_{p}^{n} \pi \nu_{\infty}\right)\right| \\
& =\left|\mathcal{F}_{q p^{n}}\left(\pi \nu_{\infty}\right)\right| \\
& \leq \underset{N}{\limsup } \mid \mathcal{F}_{q p^{n}}\left(\pi \left(\frac{1}{N} \sum_{k=1}^{N} \delta_{\left.\left.T_{p}^{k}\left(x_{\omega}\right)\right)\right) \mid}\right.\right. \\
& =\underset{N}{\limsup }\left|\mathcal{F}_{q p^{n}}\left(\frac{1}{N} \sum_{k=1}^{N} \delta_{T_{p}^{k}\left(x_{\omega}\right)}\right)\right| \\
& <\epsilon
\end{aligned}
$$

where we have made use several times of the fact that $q$ is an integer, that $\pi \nu_{\infty}$ is $\bar{T}_{p}$ invariant, and in the last line of our choice of $q$. Since $\epsilon$ was arbitrary we obtain $\mathcal{F}_{q}\left(\nu_{\infty}\right)=0$, and we are done.

Theorem 5.2, in turn, reduces to the following statement. Recall the definition of the stopping time $\tilde{\tau}$ and the random variable $\tau$ as in the beginning of Section 3.1 .

Theorem 5.3. For all $\epsilon>0$ and $\gamma^{\prime} \in(0, \gamma)$ there is $q^{*}(\epsilon, \mathbf{p}) \in \mathbb{N}$ such that for every $q \in \mathbb{Z}$ with $|q| \geq q^{*}(\epsilon, \mathbf{p})$ :

There are values $h(q, \epsilon, \mathbf{p}), k(q, \epsilon, \mathbf{p}), h^{\prime}(q, \epsilon, \mathbf{p})>0$ such that, for all $n \in \mathbb{N}$ and every $\omega \in \mathcal{A}^{\mathbb{N}}$,

$$
\mathbb{E}_{\xi \in \mathcal{A}^{h}\left(\omega^{\prime}\right)}\left|\mathcal{F}_{q s}\left(f_{\left.\xi\right|_{\tau_{k}(\xi)+\tilde{\tau}_{h^{\prime}}\left(\sigma^{\tau_{k}(\xi)}(\xi)\right)}} \circ f_{\rho_{\xi}} \nu\right)\right|^{2}+|q| e^{\left(1+\gamma^{\prime}\right) h \chi}<\epsilon
$$

Where the partition $\mathcal{A}^{h}$ is as in Definition 3.3 part (2), and: 
1. $\omega^{\prime}=\omega^{\prime}(n, \omega)=\sigma^{\tilde{\tau}_{n \log p}(\omega)}(\omega)$.

2. $s=s(\omega, n, h)=p^{n} \cdot f_{\left.\omega\right|_{\tilde{\tau}_{\frac{n \log p}{}}(\omega)} ^{\prime}}\left(f_{\left.\omega^{\prime}\right|_{\tilde{\tau}_{h}\left(\omega^{\prime}\right)}}\left(x_{0}\right)\right)$, where $x_{0} \in I$ is our prefixed point. In particular, $s, s^{-1}=O(1)$ where this $O(1)$ depends only on the IFS.

3. The word $\rho_{\xi}$ for $\xi \in \mathcal{A}^{\mathbb{N}}$ is defined as the unique word satisfying

$$
\left.\xi\right|_{\tau_{k}(\xi)+\tilde{\tau}_{h^{\prime}}\left(\sigma^{\tau_{k}(\xi)}(\xi)\right)} * \rho_{\xi}=\left.\xi\right|_{\tilde{\tau}_{h}(\xi)+\tilde{\tau}_{k+h^{\prime}-h+Q}\left(\sigma^{\tau_{h}(\xi)} \xi\right)}
$$

for some global $Q>0$ that only depends on the IFS. Furthermore, there exists some global $P>0$ such that $\left|\rho_{\xi}\right| \leq P$ for all $\xi$.

We remark that the word $\rho_{\xi}$ also depends on the parameters $k, h, h^{\prime}, Q$, but we suppress this in our notation. Both the proof of Theorem 5.3 and the proof that it implies Theorem 5.2 are not trivial. Thus, we dedicate the next Section to the proof that Theorem 5.3 implies Theorem 5.2. The subsequent Section contains the proof of Theorem 5.3. So, all in all, once these two assertions are established, Theorem 5.1 is proved and we are done.

\subsection{Proof that Theorem 5.3 implies Theorem 5.2}

\subsubsection{The martingale argument}

In this Section we employ a deep observation that was originally made by Hochman and Shmerkin [29, Theorem 2.1], and was recently further refined by Hochman [27]. Recall that if $\mathcal{C}$ is a partition of a space $X$ and $\mu$ is a probability measure on $X$, then for any $x \in \operatorname{supp}(\mu)$ we denote by $\mathcal{C}(x)$ the atom of $\mathcal{C}$ containing $x$, and by $\mu_{\mathcal{C}(x)}$ the conditional measure of $\mu$ on this atom.

Theorem 5.4. 27, Theorem 2.2] Let $T: X \rightarrow X$ be a continuous map of a compact metric space, and let $\mu$ be a Borel probability measure on $X$. Let $\left\{\mathcal{C}_{n}\right\}_{n \in \mathbb{N}}$ be a refining sequence of Borel partitions. Suppose that

$$
\lim _{k \rightarrow \infty} \sup _{n \in \mathbb{N}}\left\{\operatorname{diam}\left(T^{n} A\right): A \in \mathcal{C}_{n+k}, \mu(A)>0\right\}=0
$$

Then for $\mu$ almost every $x$,

$$
\lim _{N \rightarrow \infty}\left(\frac{1}{N} \sum_{n=1}^{N} \delta_{T^{n}(x)}-\frac{1}{N} \sum_{n=1}^{N} T^{n} \mu_{\mathcal{C}_{n}(x)}\right)=0
$$

in the weak-* sense.

That is, as long as the partitions $\left\{\mathcal{C}_{n}\right\}_{n \in \mathbb{N}}$ are compatible with the dynamics of $T$ in the sense of (48), the orbits of $\mu$ typical points are Cesàro equivalent to the $T^{n}$-magnifications of the conditionals of $\mu$ on their $\mathcal{A}^{n}$ atoms.

Now, let $0<h \ll 1$ be a fixed parameter, and consider the stopping time $\beta_{n, h}$ defined by

$$
\beta_{n, h}(\omega)=\tilde{\tau}_{\frac{n \log p}{\chi}}(\omega)+\tilde{\tau}_{h}\left(\sigma^{\frac{\tilde{\tau}_{n \log p}}{\chi}(\omega)} \omega\right) .
$$

Note that in the stopping time $\beta_{n, h}$ we let $n$ vary but keep $h$ fixed. 
Theorem 5.5. For $\mathbb{P}$ almost every $\omega$ and for every integer $q \in \mathbb{Z}$,

$$
\lim _{N \rightarrow \infty} \mathcal{F}_{q}\left(\frac{1}{N} \sum_{n=0}^{N-1} \delta_{T_{p}^{n}\left(x_{\omega}\right)}\right)-\mathcal{F}_{q}\left(\frac{1}{N} \sum_{n=0}^{N-1} T_{p}^{n} \circ f_{\left.\omega\right|_{\beta_{n, h}(\omega)}} \nu\right)=0 .
$$

Proof. Equip the compact space $X=\mathcal{A}^{\mathbb{N}} \times \mathbb{T}$ with the usual metric on each coordinate and the sup metric on the product space. For every $n$ let $\mathcal{C}_{n, h}$ be the partition of $X$ given by

$$
\begin{gathered}
\left(\omega, \pi\left(x_{\omega}\right)\right) \sim_{\mathcal{C}_{n, h}}\left(\eta, \pi\left(x_{\eta}\right)\right) \Longleftrightarrow\left(\omega_{1}, \ldots, \omega_{\beta_{n, h}(\omega)}\right)=\left(\eta_{1}, \ldots, \eta_{\beta_{n, h}(\eta)}\right), \\
\text { and if } x \neq \pi\left(x_{\omega}\right), y \neq \pi\left(x_{\eta}\right) \text { then }(\omega, x) \sim_{\mathcal{C}_{n, h}}(\eta, y) .
\end{gathered}
$$

Notice that we are grouping all the elements of $X$ that are not of the form $\left(\omega, \pi\left(x_{\omega}\right)\right)$ into a single partition cell, which we denote by $B$.

Let $\mu$ be the probability measure on $X$ defined by

$$
\mu(A)=\mathbb{P}\left(\left\{\omega: \quad\left(\omega, \pi\left(x_{\omega}\right)\right) \in A\right\}\right) .
$$

Notice that the projection of $\mu$ to $\mathbb{T}$ is $\pi \nu$, and that $\mu(B)=0$. To complete the setup, let $T: X \rightarrow X$ be the continuous map

$$
T(\omega, x)=\left(\omega, \bar{T}_{p}(x)\right) .
$$

We now verify that (48) holds true: Let $n, k \in \mathbb{N}$ and let $A \in \mathcal{C}_{n+k, h}$ be such that $\mu(A)>0$. Then for any $\omega$ with $\left(\omega, x_{\omega}\right) \in A$, recalling the metric on $\mathcal{A}^{\mathbb{N}}$ defined in Section 1.2.1,

$$
\begin{aligned}
\operatorname{diam}\left(T^{n} A\right) & =\max \left\{\operatorname{diam}\left(A_{\left.\omega\right|_{\beta_{n+k, h}(\omega)}}\right), \operatorname{diam}\left(T_{p}^{n} \circ \pi \circ f_{\left.\omega\right|_{\beta_{n+k, h}(\omega)}}(K)\right)\right\} \\
& \leq \max \left\{\rho^{\frac{\tilde{\tau}_{\log p}}{\chi}(\omega)}, p^{-k}\right\} \rightarrow 0 \text { as } k \rightarrow \infty \text { uniformly in } n \text { and } \omega .
\end{aligned}
$$

Thus, we may apply Theorem 5.4: For $\mu$ almost every $\left(\omega, \pi\left(x_{\omega}\right)\right)$ letting $\mu_{\mathcal{C}_{k, h}\left(\omega, \pi\left(x_{\omega}\right)\right)}$ be the conditional measure of $\mu$ on the atom $\mathcal{C}_{k, h}\left(\omega, x_{\omega}\right)$, we get

$$
\lim _{N \rightarrow \infty}\left(\frac{1}{N} \sum_{k=0}^{N-1} \delta_{T^{k}\left(\omega, \pi\left(x_{\omega}\right)\right)}-\frac{1}{N} \sum_{k=0}^{N-1} T^{k} \mu_{\mathcal{C}_{k, h}\left(\omega, \pi\left(x_{\omega}\right)\right)}\right)=0 .
$$

Notice that the projection of $\mu_{\mathcal{C}_{k, h}\left(\omega, \pi\left(x_{\omega}\right)\right)}$ to $\mathbb{T}$ is $\pi \circ f_{\left.\omega\right|_{\beta_{k, h}(\omega)}} \nu$. So, projecting equation (49) to $\mathbb{T}$ and using this observation, for $\mathbb{P}$ almost every $\omega$

$$
\lim _{N \rightarrow \infty}\left(\frac{1}{N} \sum_{k=0}^{N-1} \delta_{\bar{T}_{p}^{k} \circ \pi\left(x_{\omega}\right)}-\frac{1}{N} \sum_{k=0}^{N-1} \bar{T}_{p}^{k} \circ \pi \circ f_{\left.\omega\right|_{\beta_{k, h}(\omega)}} \nu\right)=0 .
$$

Finally, let $q \in \mathbb{Z}$. Invoking (47), for every $k \geq 1$,

$$
\mathcal{F}_{q}\left(\delta_{\bar{T}_{p}^{k} \circ \pi\left(x_{\omega}\right)}\right)=\mathcal{F}_{q}\left(\delta_{T_{p}^{k}\left(x_{\omega}\right)}\right) \text {, and } \mathcal{F}_{q}\left(\bar{T}_{p}^{k} \circ \pi \circ f_{\left.\omega\right|_{\beta_{k, h}(\omega)}} \nu\right)=\mathcal{F}_{q}\left(T_{p}^{k} \circ f_{\left.\omega\right|_{\beta_{k, h}(\omega)}} \nu\right) \text {. }
$$

So, combining the last two displayed equations, the Theorem is proved.

Remark 5.6. As pointed out to us by the anonymous referee, once Theorem 5.5 is established there is another way to prove Theorem 1.1 part (2): Via a slight modification of Theorem 5.5, it is enough to show that for $\nu$ as in Theorem 1.1,

$$
\text { For every } C, C_{0}>0, \quad \lim _{q} \sup \left\{\left|\mathcal{F}_{q}(g \nu)\right|: g \in C^{1+\gamma}:\|g\|_{C^{1+\gamma}}<C_{0}, \inf \left|g^{\prime}\right| \geq C_{1}\right\}=0 .
$$

That is, the rate of decay of $\left|F_{q}(g \nu)\right|$ is uniform in $g \in C^{1+\gamma}$, as long as its $C^{1+\gamma}$ norm and inf $\left|g^{\prime}\right|$ are uniformly bounded. We believe this Claim to be true, and it should follow by verifying that: 
1. The rates in Theorems 3.10 and 3.11 are uniform in $g$.

2. The other estimates as in Section 4 are uniform in $g$.

Taking this approach allows one to circumvent the use of Theorem 3.8, thus possibly shortening the proof. However, since we hope to have other applications for our method that do make use of Theorem 3.8 (e.g. for higher dimensions or dimension theory), and since it is one of the goals of this paper to show how local limit Theorems may be adapted to study the geometry of self conformal measures, we present the proof of Theorem 1.1 in its original form.

\subsubsection{First linearization and stopping time argument}

Our next step is to linearize the maps appearing in Theorem 5.5, in the following sense: Fix an integer frequency $q \neq 0 \in \mathbb{Z}$. We want to estimate $\mathcal{F}_{q}(\cdot)$ for the push-forward of $\nu$ via the map

$$
T_{p}^{n} \circ f_{\left.\omega\right|_{\beta_{n, h}(\omega)}}=T_{p}^{n} \circ f_{\omega \mid}{ }_{\tilde{\tau}_{\frac{n \log p}{\chi}}(\omega)+\tilde{\tau}_{h}(\sigma}^{\tilde{\tau}^{\left(\frac{n \log p}{\chi}\right.}{ }^{(\omega)}{ }_{\omega)}} .
$$

The idea is use the first $\tilde{\tau}_{\frac{n \log p}{\chi}}(\omega)$ digits to cancel out the $T_{p}^{n}$ factor. The price is a uniformly bounded defect $s$ in the frequency, and a controllable error term that relies on $h$ and $q$ :

Claim 5.7. (First linearization) For every $\omega \in \mathcal{A}^{\mathbb{N}}, q \neq 0 \in \mathbb{Z}, n \in \mathbb{N}$, and $h>0$ that is large enough in manner dependent only on $\gamma^{\prime} \in(0, \gamma)$

$$
\left\|\mathcal{F}_{q}\left(T_{p}^{n} \circ f_{\left.\omega\right|_{\beta_{n, h}(\omega)}} \nu\right)|-| \mathcal{F}_{q s}\left(f_{\left.\omega^{\prime}\right|_{\tilde{\tau}_{h}\left(\omega^{\prime}\right)}} \nu\right)\right\| \leq|q| e^{-\left(1+\gamma^{\prime}\right) h \chi}
$$

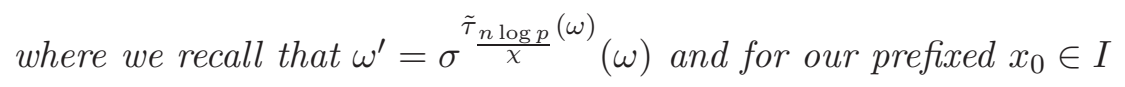

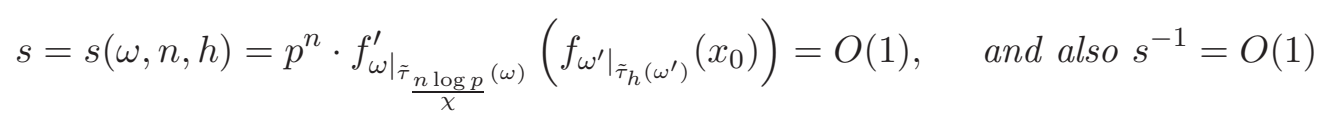

where this $O(1)$ only depends on the IFS.

Proof. We use the notation $\omega^{\prime}$ as in the statement of the Claim. Plugging in $g=f_{\left.\omega\right|_{\tilde{\tau}_{n \log p}(\omega)} ^{\chi}}$ into Lemma 2.3. as long as $h=h\left(\gamma^{\prime}\right)$ is large enough, for any $x$ and our prefixed $x_{0} \in I$

$$
\begin{aligned}
\mid f_{\left.\omega\right|_{\tilde{\tau}_{n \log p}(\omega)} ^{\chi}}\left(\left.f_{\omega^{\prime}}\right|_{\tilde{\tau}_{h}\left(\omega^{\prime}\right)}(x)\right) & -f_{\left.\omega\right|_{\tilde{\tau}_{n \log p}(\omega)} ^{\chi}}\left(\left.f_{\omega^{\prime}}\right|_{\tilde{\tau}_{h}\left(\omega^{\prime}\right)}\left(x_{0}\right)\right) \\
& -f_{\left.\omega\right|_{\tilde{\tau}_{n \log p}(\omega)} ^{\chi}}^{\prime}\left(f_{\left.\omega^{\prime}\right|_{\tilde{\tau}_{h}\left(\omega^{\prime}\right)}}\left(x_{0}\right)\right) \cdot\left(\left.f_{\omega^{\prime}}\right|_{\tilde{\tau}_{h}\left(\omega^{\prime}\right)}(x)-\left.f_{\omega^{\prime}}\right|_{\tilde{\tau}_{h}\left(\omega^{\prime}\right)}\left(x_{0}\right)\right) \mid \\
& \leq\left|f_{\left.\omega\right|_{\tilde{\tau}_{\frac{n \log p}{}}(\omega)} ^{\prime}}^{\prime}\left(f_{\left.\omega^{\prime}\right|_{\tilde{\tau}_{h}\left(\omega^{\prime}\right)}}\left(x_{0}\right)\right)\right| \cdot\left|f_{\omega^{\prime}}\right|_{\tilde{\tau}_{h}\left(\omega^{\prime}\right)}(x)-\left.\left.f_{\omega^{\prime}}\right|_{\tilde{\tau}_{h}\left(\omega^{\prime}\right)}\left(x_{0}\right)\right|^{1+\gamma^{\prime}} \\
\leq & e^{-\frac{n \log p}{\chi} \cdot \chi} \cdot e^{-h \chi\left(1+\gamma^{\prime}\right)}=p^{-n} \cdot e^{-h \chi\left(1+\gamma^{\prime}\right)}
\end{aligned}
$$

Denote $t_{0}=f_{\frac{\omega}{\left.\right|_{\tilde{\tau}_{n \log p}(\omega)} ^{\chi}}}^{\prime}\left(f_{\left.\omega^{\prime}\right|_{\tilde{\tau}_{h}\left(\omega^{\prime}\right)}}\left(x_{0}\right)\right)$ and $t_{1}=\left(1-t_{0}\right) \cdot f_{\left.\omega^{\prime}\right|_{\tilde{\tau}_{h}\left(\omega^{\prime}\right)}}\left(x_{0}\right)$. Using that $\mathcal{F}_{q}$ is $|q|$-Lipschitz and (50),

$$
\left\|\mathcal{F}_{q}\left(T_{p}^{n} \circ f_{\left.\omega\right|_{\beta_{n, h}(\omega)}} \nu\right)|-| \mathcal{F}_{q}\left(T_{p}^{n}\left(t_{0} \cdot f_{\left.\omega^{\prime}\right|_{\tau_{h}\left(\omega^{\prime}\right)}} \nu\right)\right)\right\|
$$




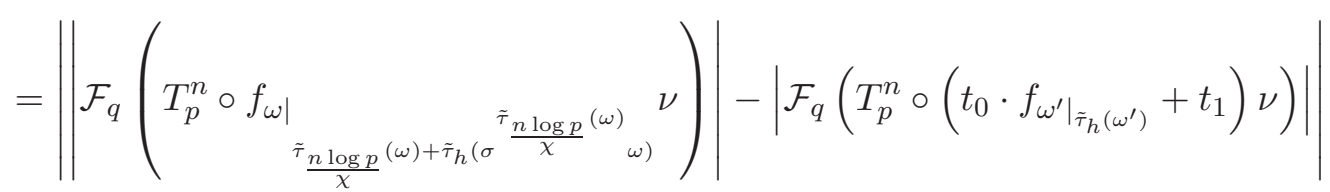

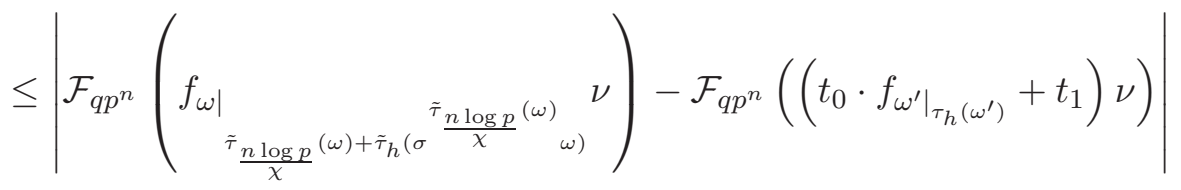

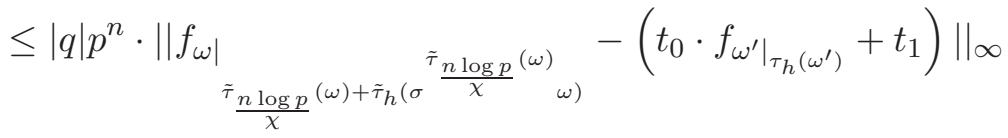

$$
\begin{aligned}
& \leq|q| p^{n} \cdot p^{-n} \cdot e^{-h \chi\left(1+\gamma^{\prime}\right)}=|q| e^{-\left(1+\gamma^{\prime}\right) h \chi} .
\end{aligned}
$$

Finally, using bounded distortion (Theorem 2.1), set $s=p^{n} t_{0}$ and note that

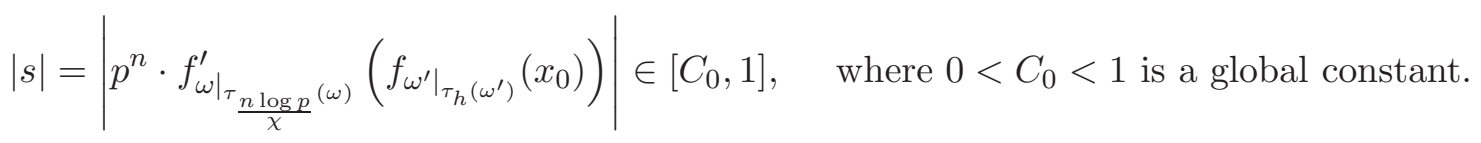

Then the result follows since, as $q$ is an integer,

$$
\left|\mathcal{F}_{q}\left(T_{p}^{n}\left(\left.t_{0} \cdot f_{\omega^{\prime}}\right|_{\tilde{\tau}_{h}\left(\omega^{\prime}\right)} \nu\right)\right)\right|=\left|\mathcal{F}_{q p^{n} t_{0}}\left(\left.f_{\omega^{\prime}}\right|_{\tilde{\tau}_{h}\left(\omega^{\prime}\right)} \nu\right)\right|=\left|\mathcal{F}_{q s}\left(\left.f_{\omega^{\prime}}\right|_{\tau_{h}\left(\omega^{\prime}\right)} \nu\right)\right| .
$$

The next Claim, which is the final ingredient in the proof that Theorem 5.3 implies Theorem 5.2, is about writing measures of the form $f_{\left.\omega\right|_{\tilde{\tau}_{h}(\omega)}} \nu$ as a certain average of measures of the form

$$
f_{\left.\xi\right|_{\tau_{k}(\xi)+\tilde{\tau}_{h^{\prime}}\left(\sigma^{\tau} k^{(\xi)}(\xi)\right)}} \circ f_{\rho_{\xi}} \nu, \quad \text { where } \xi \in \mathcal{A}^{h}(\omega), \text { and } k+h^{\prime}>h
$$

where $\rho_{\xi}$ is a word of uniformly bounded length. This is crucially important for our argument, since the local limit Theorem 3.8 applies for the random variable $\tau_{k}$, but not necessarily for the stopping time $\tilde{\tau}_{k}$.

Claim 5.8. (Relating stopping time with cocycle) There is some $P>0$ such that:

For every $\omega \in\{1, \ldots, n\}^{\mathbb{N}}, h, h^{\prime}, k>0$ with $h<k+h^{\prime}$

$$
f_{\left.\omega\right|_{\tilde{\tau}_{h}(\omega)}} \nu=\mathbb{E}_{\xi \in \mathcal{A}^{h}(\omega)}\left(f_{\left.\xi\right|_{\tau_{k}(\xi)+\tilde{\tau}_{h^{\prime}}\left(\sigma^{\tau}(\xi)(\xi)\right)}} \circ f_{\rho_{\xi}} \nu\right)
$$

where $\rho_{\xi}=\rho_{\xi, k, h, h^{\prime}, Q}$ is the unique random word satisfying

$$
\left.\xi\right|_{\tau_{k}(\xi)+\tilde{\tau}_{h^{\prime}}\left(\sigma^{\tau}(\xi)(\xi)\right)} * \rho_{\xi}=\left.\xi\right|_{\tilde{\tau}_{h}(\xi)+\tilde{\tau}_{k+h^{\prime}-h+Q}\left(\sigma^{\tau_{h}(\xi)} \xi\right)}
$$

for some global $Q>0$ that only depends on the IFS, so that $\left|\rho_{\xi}\right| \leq P$ for all $\xi \in \mathcal{A}^{\mathbb{N}}$.

Proof. Since $\nu$ is self conformal and $\tilde{\tau}$ is a stopping time, for any fixed $Q>0$ (to be chosen later)

$$
\nu=\mathbb{E}\left(f_{\left.\eta\right|_{\tilde{\tau}}+h^{\prime}-h+Q} \nu\right)
$$

so

$$
f_{\omega \mid \tilde{\tau}_{h}(\omega)} \nu=\mathbb{E}_{\eta}\left(f_{\left.\omega\right|_{\tilde{\tau}_{h}(\omega)}} \circ f_{\left.\eta\right|_{\tilde{\tau}_{k+h^{\prime}-h+Q}}} \nu\right)=\mathbb{E}_{\xi \in \mathcal{A}^{h}(\omega)}\left(f_{\left.\xi\right|_{\tilde{\tau}_{h}(\xi)}+\tilde{\tau}_{k+h^{\prime}-h+Q}\left(\sigma^{\tilde{\tau}_{h}(\xi)} \xi\right)} \nu\right)
$$


Now, by bounded distortion (Theorem 2.1), there is global $C>0$ such that for every $\xi$

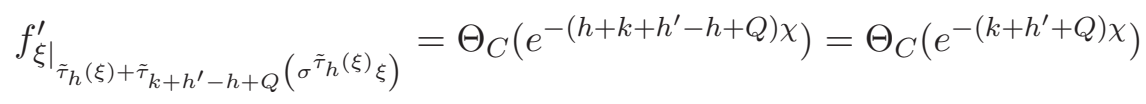

on the other hand, for every $\xi$

$$
f_{\left.\left.\xi\right|_{\tau_{k}(\xi)+\tilde{\tau}_{h^{\prime}}\left(\sigma^{\tau} k(\xi)\right.} ^{\prime}(\xi)\right)}=\Theta_{C}\left(e^{-\left(k+h^{\prime}\right) \chi}\right) .
$$

It follows that we can choose $Q$ based only on $C$ such that for every $\xi$,

$$
\tau_{k}(\xi)+\tilde{\tau}_{h^{\prime}}\left(\sigma^{\tau_{k}(\xi)}(\xi)\right) \leq \tilde{\tau}_{h}(\xi)+\tilde{\tau}_{k+h^{\prime}-h+Q}\left(\sigma^{\tilde{\tau}_{h}(\xi)} \xi\right) \leq \tau_{k}(\xi)+\tilde{\tau}_{h^{\prime}}\left(\sigma^{\tau_{k}(\xi)}(\xi)\right)+P
$$

where $P$ has uniformly finite length. Therefore, there is a word $\rho_{\xi}$ of length $\leq P$ with

$$
\left.\xi\right|_{\tau_{k}(\xi)+\tilde{\tau}_{h^{\prime}}\left(\sigma^{\tau}(\xi)(\xi)\right)} * \rho_{\xi}=\left.\xi\right|_{\tilde{\tau}_{h}(\xi)+\tilde{\tau}_{k+h^{\prime}-h+Q}\left(\sigma^{\tau_{h}(\xi)} \xi\right)} \cdot
$$

Plugging this equality into (51), the Claim is proved.

\subsubsection{Proof that Theorem 5.3 implies Theorem 5.2}

Let $\epsilon>0$. Suppose that for every integer $q$ with $|q| \geq q^{*}(\epsilon, \mathbf{p})$ there are values $h(q, \epsilon, \mathbf{p}), k(q, \epsilon, \mathbf{p})$ and $h^{\prime}(q, \epsilon, \mathbf{p})$ satisfying the conclusion of Theorem 5.3. Then, for $\mathbb{P}$ almost every $\omega$, by applying successively Theorem 5.5 and Claim 5.7 with this $h$, Claim 5.8 with these $h, h^{\prime}, k$ and $\omega^{\prime}$, Jensen's inequality, and finally Theorem 5.3 , we get

$$
\begin{aligned}
& \left|\mathcal{F}_{q}\left(\frac{1}{N} \sum_{n=1}^{N} \delta_{T_{p}^{n} x_{\omega}}\right)\right| \leq \frac{1}{N} \sum_{n=1}^{N}\left|\mathcal{F}_{q}\left(T_{p}^{n} \circ f_{\left.\omega\right|_{\beta_{n, h}(\omega)}} \nu\right)\right|+o_{N}(1) \\
& =\frac{1}{N} \sum_{n=1}^{N} \sqrt{\left|\mathcal{F}_{q}\left(T_{p}^{n} \circ f_{\left.\omega\right|_{\beta_{n, h}(\omega)}} \nu\right)\right|^{2}}+o_{N}(1) \\
& \leq \frac{\sqrt{2}}{N} \sum_{n=1}^{N} \sqrt{\left|\mathcal{F}_{q s}\left(f_{\left.\omega^{\prime}\right|_{\tau_{h}\left(\omega^{\prime}\right)}} \nu\right)\right|^{2}+|q| e^{-\left(1+\gamma^{\prime}\right) h \chi}}+o_{N}(1) \\
& =\frac{\sqrt{2}}{N} \sum_{n=1}^{N} \sqrt{\left|\mathcal{F}_{q s}\left(\mathbb{E}_{\xi \in \mathcal{A}^{h}\left(\omega^{\prime}\right)}\left(f_{\left.\xi\right|_{\tau_{k}(\xi)+\tilde{\tau}_{h^{\prime}}\left(\sigma^{\tau_{k}(\xi)}(\xi)\right)}} \circ f_{\rho_{\xi}} \nu\right)\right)\right|^{2}+|q| e^{-\left(1+\gamma^{\prime}\right) h \chi}} \\
& +\quad o_{N}(1) \\
& \leq \frac{\sqrt{2}}{N} \sum_{n=1}^{N} \sqrt{\mathbb{E}_{\xi \in \mathcal{A}^{h}\left(\omega^{\prime}\right)}\left|\mathcal{F}_{q s}\left(f_{\left.\xi\right|_{\tau_{k}(\xi)+\tilde{\tau}_{h^{\prime}}\left(\sigma^{\tau_{k}(\xi)}(\xi)\right)}} \circ f_{\rho_{\xi}} \nu\right)\right|^{2}+|q| e^{-\left(1+\gamma^{\prime}\right) h \chi}} \\
& +o_{N}(1) \\
& \leq \sqrt{2 \epsilon}+o_{N}(1) \text {. }
\end{aligned}
$$

Taking $N \rightarrow \infty$, Theorem 5.2 is proved. 


\subsection{Proof of Theorem 5.3}

The proof has two stages. First, for a fixed $\omega \in \mathcal{A}^{\mathbb{N}}$ and $n \in \mathbb{N}$, we let $q \in \mathbb{Z}$ and $h, h^{\prime}, k>0$ be arbitrary, and use them to bound

$$
\mathbb{E}_{\xi \in \mathcal{A}^{h}\left(\omega^{\prime}\right)}\left|\mathcal{F}_{q s}\left(f_{\left.\xi\right|_{\tau_{k}(\xi)+\tilde{\tau}_{h^{\prime}}\left(\sigma^{\tau}(\xi)(\xi)\right)}} \circ f_{\rho_{\xi}} \nu\right)\right|^{2}
$$

with the notation $\omega^{\prime}=\omega^{\prime}(n, \omega)$ and $s(\omega, n, h)$ as in Theorem 5.3. The resulting bound will be a sum of several error terms, depending variously on $|q|, k, h, h^{\prime}$ up to universal constants. These errors are produced by running a similar argument to the one proving Theorem 1.1 part (1) as in Section 4. In the second stage of the proof, we let $\epsilon>0$ be small and show that we may choose specific parameters $h, h^{\prime}, k$ such that all of these error terms can be made arbitrarily small simultaneously, as long as $|q|$ is large in a manner that only depends on $\epsilon$. This will give Theorem 5.3 .

\subsubsection{Collecting error terms}

Fix $\omega$ and $n$ and let $\eta$ be such that $\mathcal{A}^{h}\left(\omega^{\prime}\right)=A_{\eta}$, and let $s$ be as in Theorem 5.3. From this point forward, we can forget about $n, \omega$ and just work with the cylinder $A_{\eta}$ and the frequency qs. We do recall that $s, s^{-1}=O(1)$ uniformly in $n$ and $\omega$, and this will be used implicitly throughout the proof. Let $k, h, h^{\prime} \geq 0$ be any parameters, and let $q \in \mathbb{Z}$. For notational convenience, we assume $q \cdot s, q>0$ - otherwise, whenever they appear inside a bound, an absolute value should be applied. By Theorem 3.8, there exists a subset $\bar{A}_{k, \eta}^{h, h^{\prime}} \subseteq A_{\eta}$ such that

$$
\mathbb{P}\left(\bar{A}_{k, \eta}^{h, h^{\prime}}\right) \geq \mathbb{P}\left(A_{\eta}\right) \cdot\left(1-o_{\mathbf{p}}^{\min (h, k-h) \rightarrow \infty}(1)\right)
$$

and (ii)-(iii) of Theorem 3.8 hold for it. In particular,

$$
\begin{aligned}
& \mathbb{E}_{\xi \in \mathcal{A}^{h}\left(\omega^{\prime}\right)}\left|\mathcal{F}_{q s}\left(f_{\left.\xi\right|_{\tau_{k}(\xi)+\tilde{\tau}_{h^{\prime}}\left(\sigma^{\tau_{k}(\xi)}(\xi)\right)}} \circ f_{\rho_{\xi}} \nu\right)\right|^{2}=\mathbb{E}_{\xi \in A_{\eta}}\left|\mathcal{F}_{q s}\left(f_{\left.\xi\right|_{\tau_{k}(\xi)+\tilde{\tau}_{h^{\prime}}\left(\sigma^{\tau_{k}(\xi)}(\xi)\right)}} \circ f_{\rho_{\xi}} \nu\right)\right|^{2} \\
& \leq \int_{\xi \in \bar{A}_{k, \eta}^{h, h^{\prime}}} \mathbb{E}_{\mathcal{A}_{k}^{h, h^{\prime}}(\xi)}\left|\mathcal{F}_{q s}\left(f_{\left.\omega\right|_{\tau_{k}(\omega)+\tilde{\tau}_{h^{\prime}}\left(\sigma^{\tau} k^{(\omega)}(\omega)\right)}} \circ f_{\rho_{\omega}} \nu\right)\right|^{2} d \mathbb{P}(\xi)+o_{\mathbf{p}}^{\min (h, k-h) \rightarrow \infty}(1)
\end{aligned}
$$

Here we made use of the fact that $\left|\mathcal{F}_{q}(\cdot)\right| \leq 1$. So, our first error term is $o_{\mathbf{p}}^{\min (h, k-h) \rightarrow \infty}(1)$.

Our next step is to linearize once more, in order to set the stage for the application of the upgraded local limit Theorem 3.8 .

Claim 5.9. (Second linerization) We have

$$
\begin{gathered}
\int_{\xi \in \bar{A}_{k, \eta}^{h, h^{\prime}}} \mathbb{E}_{\mathcal{A}_{k}^{h, h^{\prime}(\xi)}}\left|\mathcal{F}_{q s}\left(f_{\left.\omega\right|_{\tau_{k}(\omega)+\tilde{\tau}_{h^{\prime}}\left(\sigma^{\tau_{k}(\omega)}(\omega)\right)}} \circ f_{\rho_{\omega}} \nu\right)\right|^{2} d \mathbb{P}(\xi) \\
\leq \int_{\xi \in \bar{A}_{k, \eta}^{h, h^{\prime}}} \mathbb{E}_{\mathcal{A}_{k}^{h, h^{\prime}}(\xi)}\left|\mathcal{F}_{q s}\left(M_{e^{-S_{\tau_{k}(\omega)}(\omega)} \circ M_{\operatorname{sign}}\left(f_{\left.\omega\right|_{\tau_{k}(x)} ^{\prime}}^{\prime}\left(x_{\sigma^{\tau} \tau_{k}(\omega)(\omega)}\right)\right.} \circ f_{\eta^{\prime}} \circ f_{\rho_{\omega}} \nu\right)\right|^{2} d \mathbb{P}(\xi) \\
+O\left(q \cdot e^{-\left(k+h^{\prime}\right) \chi_{e}} e^{-\gamma^{\prime} \cdot h^{\prime} \chi}\right)
\end{gathered}
$$

where for every $\xi \in \bar{A}_{k, \eta}^{h, h^{\prime}}$, recalling that $\mathcal{A}_{k}^{h, h^{\prime}}(\xi)=A_{k, \eta, \eta^{\prime}}, \eta^{\prime}$ is defined as $\eta^{\prime}=\eta^{\prime}(\xi)$. 
Proof. This is, up to minor changes, Claim 4.5.

So, our next error term is $q \cdot e^{-\left(k+h^{\prime}\right) \chi} e^{-\gamma^{\prime} \cdot h^{\prime} \chi}$ (up to multiplying by a global constant that we omit from notation, and recalling our assumption that $q>0$ ). With the help of the following Corollary, we can remove the randomness of the word $\rho_{\xi}$.

Corollary 5.10. Let $P>0$ be as in Claim 5.8. Then

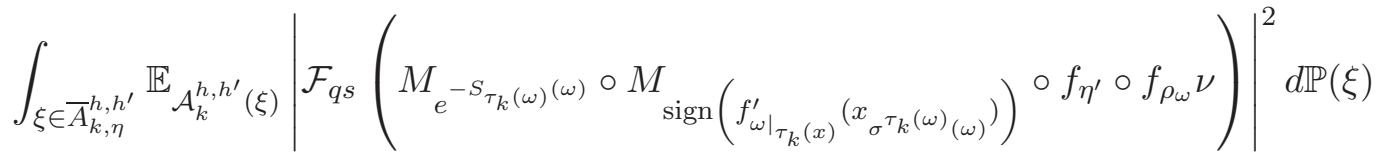

$$
\begin{aligned}
& \leq \sum_{|\rho| \leq P} \int_{\xi \in \bar{A}_{k, \eta}^{h, h^{\prime}}} \mathbb{E}_{\mathcal{A}_{k}^{h, h^{\prime}}(\xi)}\left|\mathcal{F}_{q s}\left(M_{e^{-S_{\tau_{k}}(\omega)}(\omega)} \circ f_{\eta^{\prime}} \circ f_{\rho} \nu\right)\right|^{2} d \mathbb{P}(\xi) .
\end{aligned}
$$

Recalling that $\left|\rho_{\omega}\right| \leq P$ for all $\omega$, this is analogues to Corollary 4.6 and Remark 4.7 via an assumption (without the loss of generality) that all maps are orientation preserving. We are now ready to apply Theorem 3.8

Claim 5.11. (Application of local limit Theorem) For every $\rho$ as in the sum in Corollary 5.10,

$$
\begin{gathered}
\int_{\xi \in \bar{A}_{k, \eta}^{h, h^{\prime}}} \mathbb{E}_{\mathcal{A}_{k}^{h, h^{\prime}}(\xi)}\left|\mathcal{F}_{q s}\left(M_{e^{-S_{\tau_{k}}(\omega)}}(\omega) \circ f_{\eta^{\prime}} \circ f_{\rho} \nu\right)\right|^{2} d \mathbb{P}(\omega) \\
\leq \int_{\xi \in \bar{A}_{k, \eta}^{h, h^{\prime}}} \int_{k \chi}^{k \chi+D^{\prime}}\left|\mathcal{F}_{q s}\left(M_{e^{-x}} \circ f_{\eta^{\prime}} \circ f_{\rho} \nu\right)\right|^{2} d \Gamma_{A_{k, \eta, \eta^{\prime}}(\xi)}(x) d \mathbb{P}(\xi) \\
\quad+O\left(\frac{2}{q e^{-\left(k+h^{\prime}\right) \chi}}+\left(q e^{-\left(k+h^{\prime}\right) \chi}\right)^{2} o_{\mathbf{p}}^{\min (h, k-h) \rightarrow \infty}(1)\right) .
\end{gathered}
$$

Proof. This follows from Theorem 3.8 in a similar manner to the derivation of Theorem 4.1 from Theorem 3.7 in Section 4 ,

So, the next error term added to our list is $\frac{2}{q e^{-\left(k+h^{\prime}\right) \chi}}+\left(q e^{-\left(k+h^{\prime}\right) \chi}\right)^{2} O_{\mathbf{p}}^{\min (h, k-h) \rightarrow \infty}(1)$ (again, up to a universal multiplicative constant that we ignore).

Next, using the uniform norm on the density of the measure $\Gamma_{A_{k, \eta, \eta^{\prime}}(\xi)}$ (Lemma 3.5) we get

$$
\begin{aligned}
& \sum_{|\rho| \leq 2 P} \int_{\xi \in \bar{A}_{k, \eta}^{h, h^{\prime}}} \int_{k \chi}^{k \chi+D^{\prime}}\left|\mathcal{F}_{q s}\left(M_{e^{-x}} \circ f_{\eta^{\prime}} \circ f_{\rho} \nu\right)\right|^{2} d \Gamma_{A_{k, \eta, \eta^{\prime}}(\xi)}(x) d \mathbb{P}(\xi) \\
& \leq \sum_{|\rho| \leq 2 P} \int_{\xi \in \bar{A}_{k, \eta}^{h, h^{\prime}}}\left(\int_{k \chi}^{k \chi+D^{\prime}}\left|\mathcal{F}_{q s}\left(M_{e^{-z}} \circ f_{\eta^{\prime}} \circ f_{\rho} \nu\right)\right|^{2} \cdot \frac{1}{D} d z\right) d \mathbb{P}(\xi) .
\end{aligned}
$$

So, we have reduced our problem to a sum of oscillatory integrals, that has uniformly bounded many terms. This will give rise to the final error term:

Claim 5.12. (Oscillatory integral) For every $\rho$ in the sum above, and for every $\delta>0$,

$$
\int_{\xi \in \bar{A}_{k, \eta}^{h, h^{\prime}}}\left(\int_{k \chi}^{k \chi+D^{\prime}}\left|\mathcal{F}_{q e^{s}}\left(M_{e^{-z}} \circ f_{\eta^{\prime}} \circ f_{\rho} \nu\right)\right|^{2} \cdot \frac{1}{D} d z\right) d \mathbb{P}(\xi) \leq O\left(\frac{1}{\delta q e^{-\left(k+h^{\prime}\right)}}+\sup _{y} \nu\left(B_{\delta}(y)\right)\right) .
$$

Proof. This is analogues to the application of Lemma 2.6 at the end of the proof of Theorem 1.1 part (1) in Section 4.

So, our last error term is $\frac{1}{\delta q e^{-\left(k+h^{\prime}\right)}}+\sup _{y} \nu\left(B_{\delta}(y)\right)$, for any $\delta>0$ (to be chosen later at our convenience). Yet again, we ignore the underlying global multiplicative constant. 


\subsubsection{Conclusion of the proof of Theorem 5.3}

Let us first recall the error terms we collected in the previous Section:

\section{List of error terms :}

Every bound below is in the sense of $\lesssim_{\mathbf{p}}$. Recall that $\gamma^{\prime} \in(0, \gamma)$.

Local limit Theorem (Claim 5.11), and (52)

$$
\frac{2}{q e^{-\left(k+h^{\prime}\right) \chi}}+\left(q e^{-\left(k+h^{\prime}\right) \chi}\right)^{2} o_{\mathbf{p}}^{\min (h, k-h) \rightarrow \infty}(1) ;
$$

Second linearization (Claim 5.9):

$$
q e^{-\left(k+h^{\prime}\right) \chi} e^{-\gamma^{\prime} h^{\prime} \chi}
$$

Oscillatory integral (Claim 5.12): For every $\delta>0$,

$$
\frac{1}{\delta q e^{-\left(k+h^{\prime}\right) \chi}}+\sup _{y} \nu\left(B_{\delta}(y)\right)
$$

First linearization (Claim 5.7):

$$
q e^{-\left(1+\gamma^{\prime}\right) h \chi}
$$

Our goal is to show that for every $\epsilon>0$ there is some $q^{*}(\epsilon) \in \mathbb{N}$ such that for all integer $|q| \geq q^{*}$, there exists a choice of $h, k, h^{\prime}$ based only on $\epsilon$ and $q$, so that every term in the list above is at most $\epsilon$. Recall that we are assuming, without the loss of generality, that $q>0$. We will also be minded to take care of the other constraints: $h$ needs to be large in a manner dependent on the prefixed parameter $\gamma^{\prime}$ (Claim [5.7), and $h<h^{\prime}+k$ (Claim 5.8).

\section{Choices of parameters:}

1. Fix $\delta=\delta(\epsilon, \mathbf{p})$ such that $\sup _{y} \nu\left(B_{\delta}(y)\right) \ll_{\mathbf{p}} \epsilon$. Here we use Lemma 2.2 .

2. Fix $h^{\prime}=h^{\prime}(\epsilon, \mathbf{p})$ such that $e^{-\gamma^{\prime} h^{\prime} \chi}=\delta \epsilon^{2}$.

3. Fix $h^{*}=h^{*}(\epsilon, \mathbf{p})$ such that if $\min (h, k-h) \geq h^{*}$, then the $o_{\mathbf{p}}^{\min (h, k-h) \rightarrow \infty}(1)$ term in Theorem 3.8 is $\ll \delta^{2} \epsilon^{3}$.

4. Fix $h=h(\epsilon, \mathbf{p}, q) \geq \frac{1}{\gamma^{\prime}}\left(h^{*}+h^{\triangle}\right)>h^{*}$ for some properly chosen $h^{\triangle}=h^{\triangle}(\epsilon, \mathbf{p})>0$ (arising from Claim 5.13 below) such that $q e^{-\left(1+\gamma^{\prime}\right) h \chi}=\epsilon$. This can be done if $q \geq q^{*}$ for some $q^{*}=q^{*}(\epsilon, \mathbf{p})$.

5. Fix $k=k(\epsilon, \mathbf{p}, q)$ such that $q e^{-\left(k+h^{\prime}\right) \chi}=\delta^{-1} \epsilon^{-1}$.

Claim 5.13. If $q \geq q^{*}$ for a sufficiently large $q^{*}=(\epsilon, \mathbf{p})$, then $k-h \geq h^{*}$.

Proof. We have

$$
e^{k \chi-\left(1+\gamma^{\prime}\right) h \chi}=\delta \epsilon^{2} e^{-h^{\prime} \chi}=\left(\delta \epsilon^{2}\right)^{1+\frac{1}{\gamma^{\prime}}} .
$$

Thus $k-h=\gamma^{\prime} h-O_{\epsilon, \mathbf{p}}(1)$. Denote this last $O_{\epsilon, \mathbf{p}}(1)$ by $h^{\triangle}$. Then $k-h \geq h^{*}$ following the choice of $h$ above. 
Note To employ Claim [5.8, we need that $k+h^{\prime}>h$. But this clearly follows from Claim 5.13, Also, to use Claim 5.7 we need $h$ to be large enough in a manner dependent on $\gamma^{\prime}$, but this can clearly be arranged in step (4) by potentially making $q$ larger.

With these parameters, all errors are simultaneously small:

Local limit Theorem:

$$
\frac{2}{q e^{-\left(k+h^{\prime}\right) \chi}}+\left(q e^{-\left(k+h^{\prime}\right) \chi}\right)^{2} o_{\mathbf{p}}^{\min (h, k-h) \rightarrow \infty}(1) \ll_{\mathbf{p}} \delta \cdot \epsilon+\delta^{-2} \epsilon^{-2} \cdot \delta^{2} \epsilon^{3} \leq 2 \epsilon ;
$$

Second linearization:

$$
q e^{-\left(k+h^{\prime}\right) \chi} e^{-\gamma^{\prime} h^{\prime} \chi}=\delta^{-1} \epsilon^{-1} \cdot \delta \epsilon^{2}=\epsilon
$$

Oscillatory integral:

$$
\frac{1}{\delta q e^{-\left(k+h^{\prime}\right) \chi}}+\sup _{y} \nu\left(B_{\delta}(y)\right) \ll_{\mathbf{p}} \frac{1}{\delta \cdot \delta^{-1} \epsilon^{-1}}+\epsilon \ll 2 \epsilon ;
$$

First linearization:

$$
q e^{-\left(1+\gamma^{\prime}\right) h \chi}=\epsilon
$$

Thus, recalling that

$$
\mathbb{E}_{\xi \in \mathcal{A}^{h}\left(\omega^{\prime}\right)}\left|\mathcal{F}_{q e^{s}}\left(f_{\left.\left.\xi\right|_{\tau_{k}(\xi)+\tau_{h^{\prime}\left(\sigma^{\tau}\right.}(\xi)}(\xi)\right)} \circ f_{\rho_{\xi}} \nu\right)\right|^{2}
$$

was shown to be bounded by the sum of the first three error terms mentioned above,

$$
\mathbb{E}_{\xi \in \mathcal{A}^{h}\left(\omega^{\prime}\right)}\left|\mathcal{F}_{q e^{s}}\left(f_{\left.\xi\right|_{\tau_{k}(\xi)+\tau_{h^{\prime}}\left(\sigma^{\tau}(\xi)(\xi)\right)}} \circ f_{\rho_{\xi}} \nu\right)\right|^{2}+q e^{\left(1+\gamma^{\prime}\right) h \chi} \leq C_{0} \epsilon
$$

for uniform $C_{0}$, which is what we want.

Thus, Theorem 5.3 is proved. We have shown that it implies Theorem 5.1, and since $p$ was arbitrary, this implies Theorem 1.1 part (2).

\section{Proof of Corollary 1.2}

\subsection{Proof of Corollary 1.2 part (1)}

Let $\Phi$ be a $C^{1+\gamma}$ IFS, and let $c(\cdot, \cdot)$ be the derivative cocycle. Recall that $\mathcal{A}=\{1, \ldots, n\}$ let $H^{\kappa}$ denote the space of $\kappa$-Hölder continuous maps $\mathcal{A}^{\mathbb{N}} \rightarrow \mathbb{C}$. Recall that we define

$$
\begin{gathered}
\Lambda_{c}=\left\{\theta: \exists \phi_{\theta} \in H^{\kappa} \text { with }\left|\phi_{\theta}\right|=1 \text { and } u_{\theta} \in S^{1} \text { such that } \forall(a, \omega) \in \mathcal{A} \times \mathcal{A}^{\mathbb{N}},\right. \\
\left.\phi_{\theta}\left(\iota_{a}(\omega)\right)=u_{\theta} \exp (-i \theta \cdot c(a, \omega)) \cdot \phi_{\theta}(\omega)\right\} .
\end{gathered}
$$

Following 3 Benoist and Quint [3] we say that $c$ is an aperiodic cocycle if

$$
\Lambda_{c}=\{0\}
$$

Next, writing $\Phi=\left\{f_{1}, \ldots, f_{n}\right\}$, we define

$$
F_{\Phi}=\left\{\log \left|f_{i}^{\prime}\left(y_{i}\right)\right|: \text { where } f_{i}\left(y_{i}\right)=y_{i}, \quad i \in \mathcal{A}\right\} .
$$

Notice that $F_{\Phi}$ is precisely the set that appears in Corollary 1.2 part (1).

\footnotetext{
${ }^{3}$ In fact, aperiodic cocycles are defined in [3, equation (15.8)] in a different way, by a certain spectral gap property. However, It is a consequence of [3, Lemma 15.3] that the two definitions are equivalent.
} 
Lemma 6.1. If $c$ is not aperiodic (i.e. it is periodic) then $F_{\Phi}$ belongs to a translation of a lattice.

Proof. The assumption that $c$ is not aperiodic means that there exists $0 \neq \theta \in \Lambda_{c}$. So, there exists $\phi \in H^{\kappa}$ with $|\phi|=1$ and $u \in S^{1}$ such that for all $(a, \omega) \in \mathcal{A} \times \mathcal{A}^{\mathbb{N}}$,

$$
\phi\left(\iota_{a}(\omega)\right)=u \cdot \exp (-i \theta \cdot c(a, \omega)) \cdot \phi(\omega) .
$$

Now, fix $1 \leq a \leq n$ and let $\omega=(a, a, a \ldots) \in \mathcal{A}^{\mathbb{N}}$. Plugging these into the equation above, we obtain

$$
1=u \exp \left(-i \theta \cdot\left(-\log f_{a}^{\prime}\left(x_{\omega}\right)\right)\right) .
$$

This equation implies that $F_{\Phi}$ belongs to a translation (determined by $u$ ) of the lattice $\frac{2 \pi}{\theta} \mathbb{Z}$.

Proof of Corollary 1.2 part (1) This is immediate from Lemma 6.1 and Theorem 1.1 part (1).

\subsection{Proof of Corollary 1.2 part (2)}

Let $\Phi=\left\{f_{i}(x)=r_{i} \cdot x+t_{i}\right\}_{i \in \mathcal{A}}$ be an aperiodic self-similar IFS. Recall that $\Phi$ is aperiodic if there

are $i, j \in \mathcal{A}$ such that $\frac{\log \left|r_{i}\right|}{\log \left|r_{j}\right|} \notin \mathbb{Q}$. Without the loss of generality, we assume $i=1$ and $j=2$. The following Lemma shows that from $\Phi$ we may construct an IFS $\Psi$ such that $F_{\Psi}$ does not belong to a translation of a lattice (and so its derivative cocycle is aperiodic), and such that every self similar measure with respect to $\Phi$ is also a self similar measure with respect to $\Psi$.

Lemma 6.2. Let $\Psi=\left\{f_{1} \circ f_{i}\right\}_{i \in \mathcal{A}} \bigcup\left\{f_{i}\right\}_{i=2, . ., n}$. Then:

1. The set $F_{\Psi}$ does not belong to a translation of a lattice.

2. Let $\nu$ be a self similar measure with respect to $\Phi$ and the probability vector $\mathbf{p}$. Then there exists a probability vector $\mathbf{q}$ such that $\nu$ is a self similar measure with respect to $\mathbf{q}$ and the IFS $\Psi$.

Proof. For part (1), since $\Phi$ and $\Psi$ are self similar, if $F_{\Psi}$ belongs to a translation of a lattice then for every $e_{1}, e_{2}, e_{3} \in \mathbb{R}, e_{2} \neq e_{3}$, that arise by taking the $\log$ of contraction ratios of $\Psi$, we have

$$
\frac{e_{1}-e_{2}}{e_{3}-e_{2}} \in \mathbb{Q} \text {. }
$$

So, taking $e_{1}=\log \left|r_{1} \cdot r_{1}\right|, e_{2}=\log \left|r_{2} \cdot r_{1}\right|, e_{3}=\log \left|r_{2}\right|$ we obtain

$$
\frac{\log \left|r_{1} \cdot r_{1}\right|-\log \left|r_{2} \cdot r_{1}\right|}{\log \left|r_{2}\right|-\log \left|r_{2} \cdot r_{1}\right|} \in \mathbb{Q}
$$

which implies that

$$
\frac{\log \left|r_{2}\right|}{\log \left|r_{1}\right|}-1=\frac{\log \left|r_{1}\right|-\log \left|r_{2}\right|}{-\log \left|r_{1}\right|} \in \mathbb{Q}
$$

This contradicts our assumption that $r_{1} \nsim r_{2}$, and concludes the proof of part (1).

For part (2), one may verify that $\nu$ is a self similar measure with respect to $\Psi$ and the probability vector

$$
\mathbf{q}:=\left(p_{1}^{2}, p_{1} \cdot p_{2}, \ldots, p_{1} \cdot p_{n}, p_{2}, p_{3}, \ldots, p_{n}\right)
$$

which is strictly positive since $\mathbf{p}$ is strictly positive.

We need one more standard Lemma: 
Lemma 6.3. Let $\Phi$ be an aperiodic self similar IFS on the interval $I$, and let $\Psi$ be the induced IFS as in Lemma 6.2. Let $g: I \rightarrow g(I)$ be a $C^{1+\gamma}(I)$ map with non vanishing derivative. Then every self conformal measure with respect to the conjugated IFS $g \circ \Phi \circ g^{-1}$ is also a self conformal measure with respect to the IFS $\Theta=g \circ \Psi \circ g^{-1}$. Furthermore, the derivative cocycle of the IFS $\Theta$ is aperiodic.

Proof. It is elementary that under these assumptions $F_{\Psi}=F_{\Theta}$. So, $F_{\Theta}$ equals $F_{\Psi}$ that does not lie on a translation of a lattice by Lemma 6.2 part (1), and so $F_{\Theta}$ does not lie on a translation of a lattice. By Lemma 6.1 this means that the derivative cocycle of $\Theta$ is aperiodic. The Claim about the self conformal measures is an immediate consequence of Lemma 6.2 part (2), since every self conformal measure $\nu$ with respect to $g \circ \Phi \circ g^{-1}$ is equal to $g \mu$, where $\mu$ is a self similar measure with respect to $\Phi$ with the same weights.

Finally, let $\Phi$ be an aperiodic self similar IFS and let $g: I \rightarrow g(I)$ be a $C^{1+\gamma}(I)$ map with non vanishing derivative. Then by Lemma 6.3 any self conformal measure with respect to the conjugated IFS $g \circ \Phi \circ g^{-1}$ is also a self conformal measure with respect to a uniformly contracting $C^{1+\gamma}$ IFS $\Theta$ such that its derivative cocycle is aperiodic. So, applying Theorem 1.1, this concludes the proof of the normality and the Rajchman assertions of Corollary 1.2 part (2).

\subsubsection{Proof of the quantitative assertion of Corollary 1.2 part (2)}

Here we assume that $\Phi$ is a Diophantine self similar IFS (recall the definition from (2) ). Let $\nu$ be a self similar measure with respect to the probability vector $\mathbf{p}$. Our goal is to show that there exists some $\alpha=\alpha(\nu)>0$ such that

$$
\left|\mathcal{F}_{q}(\nu)\right| \leq O\left(\frac{1}{\left.|\log | q\right|^{\alpha}}\right), \text { as }|q| \rightarrow \infty
$$

Let $\left\{r_{1}, \ldots, r_{n}\right\}$ denote the contraction ratios of $\Phi$, and let $\mu$ be the distribution $\mathbf{p}$ induces on $\left\{-\log \left|r_{1}\right|, \ldots,-\log \left|r_{n}\right|\right\}$. Our first observation is that in this case the random walk

$$
S_{n}(\omega):=-\log \left|f_{\left.\omega\right|_{n}}^{\prime}\left(x_{\sigma^{n}(\omega)}\right)\right|=-\log \left|f_{\left.\omega\right|_{n}}^{\prime}\left(x_{0}\right)\right|, \quad \text { for any prefixed } x_{0} \in I
$$

as in Section [3, is in fact a classical random on $\mathbb{R}$ and its law is given by $\mu^{* n}$, the $n$-fold self convolution of $\mu$. Since for such random walks effective versions of the central and local limit Theorems are available (which is why the Diophantine condition is imposed), we can strengthen Theorem 3.7 by specifying a rate:

Theorem 6.4. There exists some $\delta=\delta(\mathbf{p})>0$ such that for every $k, h^{\prime}>0, h=0$ and $\eta=$ the empty word:

There exists a subset $\mathcal{A}_{k, \eta}^{h, h^{\prime}} \subseteq \mathcal{A}^{\mathbb{N}}$ such that, as $k$ tends to $\infty$ :

(i) $\mathbb{P}\left(\mathcal{A}_{k, \eta}^{h, h^{\prime}}\right) \geq 1-O_{\mathbf{p}}\left(\frac{1}{k^{\delta}}\right)$.

(ii) for all $\xi \in \mathcal{A}_{k, \eta}^{h, h^{\prime}}, \mathbb{P}\left(\mathcal{A}_{k}^{h, h^{\prime}}(\xi)\right)>0$.

(iii) for all $\xi \in \mathcal{A}_{k, \eta}^{h, h^{\prime}}$ and for any sub-interval $J \subseteq\left[k \chi, k \chi+D^{\prime}\right]$,

$$
\mathbb{P}_{\mathcal{A}_{k}^{h, h^{\prime}}(\xi)}\left(S_{\tau_{k}} \in J\right)=\Gamma_{\mathcal{A}_{k}^{h, h^{\prime}}(\xi)}(J)+O_{\mathbf{p}}\left(\frac{1}{k^{\delta}}\right) .
$$


The difference between Theorem 6.4 and Theorem 3.7 is that the error term $o_{h_{0}, \mathbf{p}}^{k \rightarrow \infty}(1)=O_{\mathbf{p}}\left(\frac{1}{k^{\delta}}\right)$ is explicit (here we always take $h_{0}=0$ ). We proceed to explain how Theorem 6.4 gives us the desired logarithmic decay rate for $\mathcal{F}_{q}(\nu)$. Afterwards, we explain how to obtain Theorem 6.4 by modifying the proof of Theorem 3.7.

Proof that Theorem 6.4 implies logarithmic decay Let $\delta>0$ be as in Theorem 6.4 fix $h=0$ and let $q$ be large. Find some $C>0$ such that for every $q$ large enough there exists $k>0$ with, letting $h^{\prime}=\sqrt{k}$,

$$
|q|=\Theta_{C}\left(k^{\frac{\delta}{4}} \cdot e^{\left(k+h^{\prime}\right) \chi}\right) .
$$

Notice that asymptotically $k \approx \log |q|$. Following the argument in Section 4, we bound $\left|\mathcal{F}_{q}(\nu)\right|$ by the sum of the following terms. As usual, every bound below is in the sense of $\lesssim_{\mathbf{p}}, 0<\gamma^{\prime}<1, q$ is assumed to be positive, and we ignore global multiplicative constants.

Linearization - Claim 4.5 (note that in the self-similar case this step can be easily bypassed, but for consistency we still take this term into account) :

$$
q e^{-\left(k+h^{\prime}\right) \chi} e^{-\gamma^{\prime} h^{\prime} \chi}
$$

Local limit Theorem - proof of Theorem 4.1 and the discussion following Corollary 4.6

$$
\frac{2}{q e^{-\left(k+h^{\prime}\right) \chi}}+\left(q e^{-\left(k+h^{\prime}\right) \chi}\right)^{2} \frac{1}{k^{\delta}}
$$

Here in equation (32) in the proof of Theorem 4.1 we use Theorem 6.4 instead of Theorem 3.7

Oscillatory integral: For every $r>0$,

$$
\frac{1}{r q e^{-\left(k+h^{\prime}\right) \chi}}+\sup _{y} \nu\left(B_{r}(y)\right)
$$

Choice of parameters : Recall that $h^{\prime}=\sqrt{k}$ and fix $r=k^{\frac{-\delta}{8}}$. Then we get:

linearization:

$$
q e^{-\left(k+h^{\prime}\right) \chi} e^{-\gamma^{\prime} h^{\prime} \chi}=k^{\frac{\delta}{4}} \cdot e^{-\gamma^{\prime} \sqrt{k} \chi}, \quad \text { This decays exponentially fast in } k .
$$

Local limit Theorem:

$$
\frac{2}{q e^{-\left(k+h^{\prime}\right) \chi}}+\left(q e^{-\left(k+h^{\prime}\right) \chi}\right)^{2} \frac{1}{k^{\delta}}=\frac{2}{k^{\frac{\delta}{4}}}+k^{\frac{\delta}{2}} \cdot \frac{1}{k^{\delta}}, \quad \text { This decays polynomially fast in } k .
$$

Oscillatory integral: There is some $d>0$ such that

$$
\frac{1}{r q e^{-\left(k+h^{\prime}\right) \chi}}+\sup _{y} \nu\left(B_{r}(y)\right) \leq \frac{k^{\frac{\delta}{8}}}{k^{\frac{\delta}{4}}}+k^{\frac{-d \cdot \delta}{8}}, \quad \text { This decays polynomially fast in } k .
$$

Here we made use of [25, Proposition 2.2], where it is shown that there is some $C>0$ such that for every $r>0$ small enough

$$
\sup _{y} \nu\left(B_{r}(y)\right) \leq C r^{d}
$$

Finally, by summing these error terms we see that for some $\alpha=\alpha(\nu)>0$ we have $\left|\mathcal{F}_{q}(\nu)\right|=O\left(\frac{1}{k^{\alpha}}\right)$. Since $k \approx \log |q|$ our claim follows.

Proof of Theorem 6.4 Recall that here $S_{n} \sim \mu^{* n}$. Thus, we follow the proof of Theorem 3.7 essentailly verbatim, only in the proof Proposition 3.12 we use the effective Berry-Esseen inequality 
[23] instead of Theorem 3.10, and in the proof of Proposition 3.13 we use Breuillard's effective local limit Theorem [7, Théorème 4.2] instead of Theorem 3.11. Indeed, recall that the Berry-Esseen inequality yields a rate of $O_{\mathbf{p}}\left(\frac{1}{\sqrt{n}}\right)$ in the central limit Theorem for $S_{n}$. So, it is straightforward to see that applying the Berry-Esseen inequality instead of Theorem 3.10 in the proof of Proposition 3.12 yields that the error term $o_{h_{0}, \mathbf{p}}^{k \rightarrow \infty}(1)=o_{0, \mathbf{p}}^{k \rightarrow \infty}(1)$ decays polynomially in $k$. As for Proposition 3.13. we require the following Theorem of Breuillard. Recall that we are assuming $\Phi$ is Diophantine in the sense that (2) holds true.

Theorem 6.5. 7, Théorème 4.2 and Remarque 4.1] There exists a sequence $\epsilon_{n}(\mu)$ and some $\delta>0$ such that $\epsilon_{n}=o\left(n^{-\delta}\right)$ and the centred distribution $\theta$, where $\theta(A):=\mu(A-\chi)$, satisfies:

Let $r>0$ denote the variance of the Gaussian on $\mathbb{R}$ associated with $\mu$ in the CLT, and fix $R>0$. For $x \in \mathbb{R}, s>0$ and the interval $I=I_{s}=[-s, s]$ we have, for $n \in \mathbb{N}$,

$$
\sup \left\{\left|\frac{1}{G_{\sqrt{n} r}(x)} \theta^{* n}(I+x)-\lambda\left(I_{s}\right)\right|:|x|+s \leq \sqrt{R n \log n}, s>n^{-\delta}\right\} \leq \epsilon_{n}(\mu)
$$

where we recall that $G_{t}(\cdot)$ stands for the density of the Gaussian law $N\left(0, t^{2}\right)$.

We remark that the statement of [7, Théorème 4.2] does not include $G_{t}$, but this version of the Theorem follows easily by combining it with the arguments of Benoist-Quint as in [3, Section 16.3], specifically with [3, Lemma 16.13]. Now, fix $\delta$ as in Theorem 6.5. Instead of using Theorem 3.11 in equation (19), we use Theorem 6.5 and translates $W$ of diameter $n^{\frac{-\delta}{4}}$, so that the error term $o_{\epsilon, h_{0}, \mathbf{p}}^{n \rightarrow \infty}(1)$ becomes the polynomially decaying $\epsilon_{n}(\mu)$. Noting that Lemma 3.14 also holds with a polynomial rate, it is now a straightforward versification that Proposition 3.13 holds with a polynomial rate. Theorem 6.4 is proved.

\subsection{Some examples of Diophantine IFS's}

Let $\Phi$ be an orientation preserving self similar IFS with contraction ratios $\left\{r_{1}, \ldots, r_{n}\right\}$. Li and Sahlsten [40, Theorem 1.3] proved that every self similar measure has logarithmic Fourier decay if the following condition holds:

There exist $C>0, l>2$ and $r_{i}, r_{j}$ such that $\left|\frac{\log r_{i}}{\log r_{j}}-\frac{p}{q}\right| \geq \frac{C}{q^{l}}$ for all $p \in \mathbb{Z}$ and $q \in \mathbb{N}$.

Recall our Diophantine condition from (2). In this Section we will indicate a family of IFS's that are Diophantine in the sense of (2) but not in the sense of (53).

To this end, notice that any rational number fails the condition (53), and recall that an irrational number is called Liouville if it fails (53). So, to produce the desired examples it is clearly sufficient to find sets $\left\{v_{1}, \ldots, v_{n}\right\}$ of strictly positive real numbers such that:

(i) For every $i, j$ we have that $\frac{v_{i}}{v_{j}}$ is either rational or Liouville.

(ii) There are $l, C>0$ such that

$$
\inf _{y \in \mathbb{R}} \max _{i \in\{1, \ldots n\}} d\left(v_{i} \cdot x+y, \mathbb{Z}\right) \geq \frac{C}{|x|^{l}}, \text { for all } x \in \mathbb{R} \text { large enough in absolute value. }
$$

We require the following Theorem of Moser [42]: 
Theorem 6.6. 42, Theorem 2] For every $n \geq 2$ and $\tau>\frac{2}{n-1}$ there exists a set of the cardinality of the continuum of vectors $\left(\alpha_{1}, \ldots, \alpha_{n}\right) \in \mathbb{R}^{n}$ such that:

1. There exists some $D>0$ such that for every $q \in \mathbb{N}$ we have

$$
\max _{i=1, \ldots, n} d\left(q \cdot \alpha_{i}, \mathbb{Z}\right) \geq D \cdot q^{-\tau} .
$$

2. For every linearly independent vectors $g, h \in \mathbb{Z}^{n+1}$

$$
\text { The ratio } \frac{g_{0}+g_{1} \alpha_{1}+\ldots+g_{n} \alpha_{n}}{h_{0}+h_{1} \alpha_{1}+\ldots+h_{n} \alpha_{n}} \text { is a Liouville number. }
$$

We proceed to construct $\left\{v_{1}, \ldots, v_{4}\right\}$ with properties (i) and (ii) as above. Let $\tau>2$ and find $\left(\alpha_{1}, \alpha_{2}\right)$ as in Theorem 6.6. Assuming without the loss of generality that both $\alpha_{1}, \alpha_{2}>0$ we define

$$
v_{1}=1, \quad v_{2}=2, \quad v_{3}=\alpha_{1}+1, \quad v_{4}=\alpha_{2}+1
$$

(if $\alpha_{i}<0$ for $i=1,2$ we define $v_{i+2}=1-\alpha_{i}$ and proceed with the same proof). By Theorem 6.6 part (2) for every $i, j$ the ratio $\frac{v_{i}}{v_{j}}$ is either rational or Liouville. Therefore, (i) holds. To verify (ii), let $|x| \gg 1$ and $y \in \mathbb{R}$. We may assume that

$$
d\left(x v_{i}+y, \mathbb{Z}\right)<\frac{1}{|x|^{2 \tau}}, \quad i=1,2 .
$$

So for $i=1,2$ there are $n_{i} \in \mathbb{Z}$ and $\epsilon_{i}$ with $\left|\epsilon_{i}\right|<\frac{1}{|x|^{2 \tau}}$ such that

$$
x v_{i}+y=n_{i}+\epsilon_{i} .
$$

Note that this implies $x=\frac{\left(n_{2}-n_{1}\right)+\left(\epsilon_{2}-\epsilon_{1}\right)}{v_{2}-v_{1}}=\left(n_{2}-n_{1}\right)+\left(\epsilon_{2}-\epsilon_{1}\right)$. So, for $i=3,4$ and any $k \in \mathbb{Z}$

$$
\begin{aligned}
\left|x v_{i}+y+k\right| & =\left|x\left(v_{i}-v_{1}\right)+n_{1}+k+\epsilon_{1}\right| \\
& =\left|\left(n_{2}-n_{1}\right) \frac{v_{i}-v_{1}}{v_{2}-v_{1}}+n_{1}+k+\frac{\epsilon_{2}-\epsilon_{1}}{v_{2}-v_{1}}\right| \\
& \geq\left|\left(n_{2}-n_{1}\right) \frac{v_{i}-v_{1}}{v_{2}-v_{1}}+n_{1}+k\right|-\frac{2}{|x|^{2 \tau}} \\
& \geq d\left(\left(n_{2}-n_{1}\right) \frac{v_{i}-v_{1}}{v_{2}-v_{1}}, \mathbb{Z}\right)-\frac{2}{|x|^{2 \tau}} .
\end{aligned}
$$

Finally since $\frac{v_{i}-v_{1}}{v_{2}-v_{1}}=\alpha_{i-2}$ for $i=3,4$, and $|x| \geq C_{0}\left|n_{2}-n_{1}\right|$ for some global $C_{0}>0$ as long as $|x|$ is large enough, by part (1) of Theorem 6.6 we obtain:

$$
\begin{aligned}
\max _{i=3,4} d\left(v_{i} \cdot x+y, \mathbb{Z}\right) & \geq \max _{i=3,4} d\left(\left(n_{2}-n_{1}\right) \frac{v_{i}-v_{1}}{v_{2}-v_{1}}, \mathbb{Z}\right)-\frac{2}{|x|^{2 \tau}} \\
& =\max _{i=1,2} d\left(\left(n_{2}-n_{1}\right) \alpha_{i}, \mathbb{Z}\right)-\frac{2}{|x|^{2 \tau}} \\
& \geq \frac{D}{\left|n_{2}-n_{1}\right|^{\tau}}-\frac{2}{|x|^{2 \tau}} \geq \frac{C^{\prime}}{|x|^{\tau}} .
\end{aligned}
$$

For some global constant $C^{\prime}$, as long as $|x|$ is large enough. This proves (ii) for $\left\{v_{1}, \ldots, v_{4}\right\}$ as claimed. Finally, notice that essentially the same proof yields a wide class of further examples of Diophantine IFS's satisfying (2) but not (53) with arbitrarily many contraction ratios (in particular, more than 4). 
Remark 6.7. As pointed out to us by the anonymous referee, if $\Phi$ satisfies that there are $l, C>0$ such that

$$
\max _{i \in\{1, \ldots n\}} d\left(\log \left|r_{i}\right| \cdot x, \mathbb{Z}\right) \geq \frac{C}{|x|^{l}}, \text { for all } x \in \mathbb{R} \text { large enough in absolute value }
$$

then the induced IFS $\Psi$ as in Lemma 6.2 is Diophantine in the sense of (2). In general, however, (54) does not imply (2) (but the converse is obviously true). To see this, let $x, y \in \mathbb{R}$ be such that $y$ is a Liouville number and $x$ is a Diophantine number in the sense of [40, Equation (1.2)]. Then the vector $v=(1, x, 1+(x-1) y)$ fails (2) : Indeed, (2) holds for $v=(x-1) \cdot(0,1, y)+(1,1,1)$ if and only if it holds for $(0,1, y)$, and clearly $(0,1, y)$ fails (2). On the other hand, it can be shown that $v$ satisfies (54) since $x$ is Diophantine.

The main reason why we work with (21) rather than (54) is that in an upcoming paper we will show that a certain analogue of it for $C^{1+\gamma}$ non-linear IFS's implies effective Fourier decay for self conformal measures. In this non-linear setting it is not clear that if we assume an analogue of (54) then the induced IFS as in Lemma 6.2 will satisfy the needed condition. We also note that (2) is consistent with the related works [7, 20, 1] that we either make use of here, or plan to make use of in future works.

\subsection{Proof of Corollary 1.2 part (3)}

Let $\Phi=\left\{f_{1}, \ldots, f_{n}\right\}$ be a uniformly contracting $C^{r}$ smooth IFS on the interval $I$, where either $r=2$ or $r=\omega$. Suppose that there exists a self conformal measure $\nu$ that is not pointwise absolutely normal or not Rajchman. We can define a derivative cocycle $c^{\prime}$ directly on $\mathcal{A} \times I$ by

$$
c^{\prime}(i, x)=-\log \left|f_{i}^{\prime}(x)\right|
$$

Notice that, unlike the symbolic version we have been working with so far, here we need $\Phi$ to be at least $C^{2}$ so that the cocycle $c^{\prime}$ has a finite moment in the sense of [3, Equation (11.15)]. This also implies for every fixed $i$ the cocycle $c^{\prime}$ is $C^{1}$ and therefore Lipschitz continuous in $x \in I$. Let $L$ denote the space of Lipschitz continuous maps $I \rightarrow \mathbb{C}, K$ be the attractor of $\Phi$, and define

$$
\begin{gathered}
\Lambda_{c^{\prime}}=\left\{\theta: \exists \phi_{\theta} \in L \text { with }\left|\phi_{\theta}\right|=1 \text { and } u_{\theta} \in S^{1} \text { such that } \forall(a, x) \in \mathcal{A} \times K,\right. \\
\left.\phi_{\theta}\left(f_{a}(x)\right)=u_{\theta} \exp \left(-i \theta \cdot c^{\prime}(a, x)\right) \cdot \phi_{\theta}(x)\right\} .
\end{gathered}
$$

Claim 6.8. The assumptions of Corollary 1.2 part (3) imply that $\Lambda_{c^{\prime}} \neq\{0\}$.

Proof. If $\Lambda_{c^{\prime}}=\{0\}$ then the cocycle $c^{\prime}$ is aperiodic. Therefore Benoist and Quint's local limit Theorem for cocycles [3, Theorem 16.15] applies to $c^{\prime}$. The crucial observation here is that, starting from a given point $x_{0} \in I$, the $n$-step random walk driven by the cocycle $c$ and the $n$-step random walk driven by the cocycle $c^{\prime}$ have exactly the same law, which is the corresponding push-forward of the distribution of $\mathbb{P}$ on the first $n$ digits. It follows that [3, Theorem 16.15] applies to $c$ as well, as long as the target $\varphi=1_{A_{\eta}}$ for some cylinder $A_{\eta}$ (to see this, just work with the the conjugated IFS $f_{\eta} \circ \Phi \circ f_{\eta}^{-1}$ for which $f_{\eta} \nu$ is self conformal). Therefore, Theorem 3.11 holds true as stated. Since this is the only use we make of the assumption $\Lambda_{c}=\{0\}$, it follows from the proof of Theorem 1.1 that $\nu$ is both Rajchman and pointwise absolutely normal. This contradicts our assumptions.

So, there exists $0 \neq \theta \in \Lambda_{c^{\prime}}$. The next Lemma now follows via a standard argument: 
Lemma 6.9. If $\Phi$ is $C^{2}$ then there exists a $C^{1}$ function $\varphi: I \rightarrow \mathbb{R}$ and some $\alpha \in \mathbb{R}$ such that for $\operatorname{every}(i, x) \in \mathcal{A} \times K$,

$$
\varphi\left(f_{i}(x)\right)=\alpha+\theta \cdot\left(-\log \left|f_{i}^{\prime}(x)\right|\right)+\varphi(x) \bmod 1 .
$$

Furthermore, if $\Phi$ is $C^{\omega}$ then we may assume $\varphi$ is also $C^{\omega}$.

The next Lemma is where the assumption that $K$ is an interval when $\Phi$ is $C^{2}$ comes into play:

Lemma 6.10. Let $\varphi$ and $\alpha$ be as in Lemma 6.9. If $\Phi$ is $C^{2}$ and $K$ is an interval then for every $1 \leq i \leq n$ there is some $n_{i} \in \mathbb{Z}$ such that for every $x \in I$

$$
\varphi\left(f_{i}(x)\right)=\alpha+\theta \cdot\left(-\log \left|f_{i}^{\prime}(x)\right|\right)+\varphi(x)+n_{i} .
$$

If $\Phi$ is $C^{\omega}$ then every $y \in K$ admits a neighbourhood $N_{y}$ in $I$ and $n_{y, i} \in \mathbb{Z}$ such that for every $x \in N_{y}$ and every $i$

$$
\varphi\left(f_{i}(x)\right)=\alpha+\theta \cdot\left(-\log \left|f_{i}^{\prime}(x)\right|\right)+\varphi(x)+n_{y, i} .
$$

Notice that one difference between this Lemma and Lemma 6.9 is the set on which the cohomological equation holds.

Proof. First, by Lemma 6.9, for every $1 \leq i \leq n$ and for all $x \in K$

$$
\varphi\left(f_{i}(x)\right)-\left(\alpha+\theta \cdot\left(-\log \left|f_{i}^{\prime}(x)\right|\right)+\varphi(x)\right) \in \mathbb{Z} .
$$

Assuming $K$ is an interval, the function on the left hand side is a continuous function taking values in $\mathbb{Z}$, so it must be constantly $n_{i} \in \mathbb{Z}$ on $K$.

If $\Phi$ is $C^{\omega}$ then so is $\varphi$. So, the function on the left hand side in the last displayed equation is a $C^{\omega}$ function on $K$ that takes values in $\mathbb{Z}$. Since $K$ is compact and infinite, this Lemma follows.

Proof of Corollary 1.2 part (3) Assume first that $\Phi$ is $C^{2}$ smooth and that $K$ is an interval. Let $\varphi$ be as in Lemma 6.10, and let $h: I \rightarrow \mathbb{R}$ be a $C^{2}$ smooth function that is a primitive of $\exp \left(\frac{\varphi(x)}{\theta}\right)$ on $I$. Now, for every $i$ define

$$
g_{i}(x)=h \circ f_{i} \circ h^{-1}: h(I) \rightarrow h(I)
$$

and let $\Psi$ be the IFS consisting of the maps $g_{i}$. We claim that $\Psi$ is a periodic self similar IFS. Indeed, by Lemma 6.10, for every $i$ and every $y \in h(I)$

$$
\begin{aligned}
g_{i}^{\prime}(y) & =\left(h \circ f_{i} \circ h^{-1}\right)^{\prime}(y) \\
& =\frac{h^{\prime}\left(f_{i} \circ h^{-1}(y)\right) \cdot f_{i}^{\prime}\left(h^{-1}(y)\right)}{h^{\prime}\left(h^{-1}(y)\right)} \\
& \left.=\exp \left(\frac{\varphi \circ f_{i}\left(h^{-1}(y)\right)}{\theta}+\log \left|f_{i}^{\prime}\left(h^{-1}(y)\right)\right|\right)-\frac{\varphi\left(h^{-1}(y)\right)}{\theta}\right) \cdot \operatorname{sign}\left(f_{i}^{\prime}\left(h^{-1}(y)\right)\right) \\
& =\exp \left(\frac{n_{i}}{\theta}+\frac{\alpha}{\theta}\right) \cdot \operatorname{sign}\left(f_{i}^{\prime}\left(h^{-1}(y)\right)\right)
\end{aligned}
$$

Since by uniform contraction $\operatorname{sign}\left(f_{i}^{\prime}\left(h^{-1}(y)\right)\right)$ is constant in $y, \Psi$ is a self similar IFS. Finally, $\Psi$ must be periodic, since if it were aperiodic then by Corollary 1.2 part (2) the measure $\nu$ would be both pointwise absolutely normal and Rajchman, contradicting our assumptions.

If the IFS is $C^{\omega}$ smooth then the same proof shows that $h(K)$ can be covered by finitely many intervals on which every map in $\Psi$ acts as an affine map, with contraction ratios of the form $r^{n_{i}}$. We leave the verification to the reader. 


\section{Proof of Theorem 1.3}

\subsection{Proof of Theorem 1.4}

In this Section we prove Theorem 1.4, which is the key to the proof of Theorem 1.3. We follow the same notations as before: Let $\Phi=\left\{f_{1}, \ldots, f_{n}\right\}$ be a self-similar IFS, and let $\nu$ be a self similar measure. Let $p \geq 2$ be an integer, and define a stopping time

$$
\beta_{n}(\omega):=\min \left\{m:\left|f_{\left.\omega\right|_{m}}^{\prime}(0)\right|<e^{-n \cdot \log p}\right\} .
$$

The following is a complete analogue of Theorem 5.5

Theorem 7.1. For $\mathbb{P}$ almost every $\omega$, for every integer $q$

$$
\lim _{N \rightarrow \infty} \mathcal{F}_{q}\left(\frac{1}{N} \sum_{n=0}^{N-1} \delta_{T_{p}^{n}\left(x_{\omega}\right)}\right)-\mathcal{F}_{q}\left(\frac{1}{N} \sum_{n=0}^{N-1} T_{p}^{n} \circ f_{\left.\omega\right|_{\beta_{n}(\omega)}} \nu\right)=0 .
$$

Recall that our aim is to prove the following Theorem:

Theorem 7.2. If $\nu$ is a Rajchman measure then it is pointwise normal to base $p$.

We need the following key Proposition.

Proposition 7.3. For every $\epsilon>0$ there is some $q_{0}=q_{0}(\epsilon)$ such that for all integer $|q|>q_{0}$, for $\mathbb{P}$ almost every $\omega$ and every $n$,

$$
\left|F_{q}\left(T_{p}^{n} \circ f_{\left.\omega\right|_{\beta_{n}(\omega)}} \nu\right)\right|<\epsilon .
$$

Proposition 7.3 implies Theorem 7.2 , By appealing first to Theorem 7.1 and using the relation between $\mathbb{P}$ and $\nu$, this is completely analogous to the implication Theorem $5.2 \Rightarrow$ Theorem 5.1 , Proof of Proposition 7.3 Let $\epsilon>0$ and let $q \in \mathbb{Z}$. Fix $\omega$, a $\mathbb{P}$ typical point. For every $n$ and $x \in K$, since $\Phi$ is a self similar IFS (and so we may assume all maps are defined on $\mathbb{R}$ )

$$
T_{p}^{n} \circ f_{\left.\omega\right|_{\beta_{n}(\omega)}}(x)=p^{n}\left(f_{\left.\omega\right|_{\beta_{n}(\omega)}}^{\prime}(0) \cdot x+f_{\left.\omega\right|_{\beta_{n}(\omega)}}(0)\right)-m_{x, n}, \quad \text { where } m_{x, n} \in \mathbb{Z} .
$$

Note: There is some global $C_{0}>0$ such that $\left|p^{n} \cdot f_{\left.\omega\right|_{\beta_{n}(\omega)}}^{\prime}(0)\right| \in\left[C_{0}, 1\right]$ for all $n$ and $\omega$. Let $r(\omega, n)=p^{n} \cdot f_{\left.\omega\right|_{\beta_{n}(\omega)} ^{\prime}}^{\prime}(0)$. Then, by (55) and since $q \in \mathbb{Z}$

$$
\left|F_{q}\left(T_{p}^{n} \circ f_{\left.\omega\right|_{\beta_{n}(\omega)}} \nu\right)\right|=\left|F_{q \cdot r(\omega, n)}(\nu)\right| .
$$

Since $r(\omega, n)$ is now fixed and its norm is in $\left[C_{0}, 1\right]$, and since $\nu$ is assumed to be Rajchman, for all $|q|>q_{0}\left(\epsilon, C_{0}\right)=q_{0}(\epsilon)$ and every $\omega, n$

$$
\left|F_{q \cdot r(\omega, n)}(\nu)\right|<\epsilon
$$

The proof is complete. 


\subsection{Reduction to IFS's in integer form}

We begin the proof of Theorem 1.3. Fix the self similar IFS $\Phi=\left\{f_{i}(x)=a_{i} x+b_{i}\right\}$, where $a_{i}>0$ for all $i$. Recall the notation $\Phi_{1}:=\Phi$ and for every integer $m \geq 2$,

$$
\Phi_{m}:=\left\{g: g=\phi_{1} \circ \ldots \circ \phi_{\ell}, \text { such that } \phi_{i} \in \Phi, g^{\prime}(0)<\frac{1}{m} \text { and }\left(\phi_{1} \circ \ldots \circ \phi_{\ell-1}\right)^{\prime}(0) \geq \frac{1}{m}\right\}
$$

and that for every $m \in \mathbb{N}, K_{\Phi_{m}}=K_{\Phi}$, i.e. all these IFS's have the same attractor as $\Phi$. Let $K$ denote this common attractor. We require the following Claim:

Claim 7.4. $\operatorname{dim} K=\sup \left\{\operatorname{dim} \mu: \mu\right.$ is a self similar measure w.r.t $\left.\Phi_{m}, m \in \mathbb{N}\right\}$.

Recall that in this paper self similar measures always correspond to a strictly positive probability vector on the underlying IFS. While we have not been able to find the statement of Claim 7.4 in the literature, it can nonetheless be inferred from a combination of existing results. We thus postpone its proof to Section 7.4 .

It will be convenient to introduce the notation

$$
\mathbb{A}=\{x: x \text { is absolutely normal }\} .
$$

So, if

$$
\operatorname{dim} K \cap \mathbb{A}<\operatorname{dim} K
$$

then by Claim 7.4 there is some IFS $\Phi_{m}$ and a self similar measure $\mu$ on it that is not pointwise absolutely normal. Without the loss of generality, we assume that $m=1$. That is, $\Phi$ already admits such a self similar measure. Notice that if there is a self similar measure $\mu$ with $\operatorname{dim} \mu=\operatorname{dim} K$ then $\mu$ cannot be pointwise absolutely normal, and we can work with this measure.

So, let $\mu$ be a non pointwise absolutely normal self similar measure. We first claim that it is impossible that there exist contraction ratios $a_{i}, a_{j}$ of maps in $\Phi$ such that $\frac{\log a_{i}}{\log a_{j}} \notin \mathbb{Q}$. Indeed, if this is the case then it follows from [40, Theorem 1.2] that $\mu$ is a Rajchman measure. So, by Theorem $1.4 \mu$ is pointwise absolutely normal, a contradiction.

Therefore, we may assume that every $a_{i}$ is a rational power of some $r>0$. Since $\mu$ cannot be a Rajchman measure, following the work of Brémont [6, Theorem 2.3] the IFS $\Phi$ is affinely conjugated via a map $h$ to an IFS $\Psi=\left\{g_{i}(x)=r_{i} x+t_{i}\right\}$ that is in so-called Pisot form [6. Definition 2.2]. For the time being, we note that this means that for every $g_{i} \in \Psi, g_{i}(x)=r^{n_{i}} x+t_{i}$ where $r^{-1}$ is a Pisot number, and $\left(n_{i}\right)$ are relatively prime (the $t_{i}$ also have an explicit form, but we postpone discussion about this to the next section).

We next claim that $r^{-1}$ is an integer. Again, we argue via contradiction: Otherwise, $r^{-1}$ is a non-integer Pisot number, which in particular implies that $r^{-1}$ is independent of all integers $p \geq 2$. We require the following well known Lemma, which is adapted from e.g. the work of Shmerkin-Peres [45, Proposition 6] and Shmerkin [57, Lemma 4.2]:

Lemma 7.5. For every $\epsilon>0$ there exists an IFS $\Lambda$ satisfying the following properties:

1. Every $f \in \Lambda$ is a composition of maps from $\Phi$.

2. Every $f \in \Lambda$ has the same contraction ratio, which must be of the form $r^{-k}$ for some $k \in \mathbb{N}$ by the structure of $\Phi$ and (1).

3. $\Lambda$ satisfies the strong separation condition: The union

$$
K_{\Lambda}=\bigcup_{f \in \Lambda} f\left(K_{\Lambda}\right)
$$

is disjoint. In particular, the IFS $\Lambda$ is regular. 


\section{4. $K_{\Lambda} \subseteq K$ and we have $\operatorname{dim} K \leq \operatorname{dim} K_{\Lambda}+\epsilon$.}

Now, let $\epsilon>0$ and produce an IFS $\Lambda$ satisfying the conditions of Lemma 7.5, It is well known that for such homogeneous IFS's with separation there exists a self similar measure $\nu$ on $K_{\Lambda}$ such that $\operatorname{dim} \nu=\operatorname{dim} K_{\Lambda}$. By [29, Theorem 1.4], since the IFS $\Lambda$ is regular and $r^{-1}$ is independent of all integer $p \geq 2, \nu$ a.e. $x$ is normal to all integer bases, that is, $\nu(\mathbb{A})=1$. Since $K_{\Lambda} \subseteq K$, we find that

$$
\operatorname{dim} \mathbb{A} \cap K \geq \operatorname{dim} \mathbb{A} \cap K_{\Lambda} \geq \operatorname{dim} \nu=\operatorname{dim} K_{\Lambda} \geq \operatorname{dim} K-\epsilon .
$$

Taking $\epsilon \rightarrow 0$ we find that $\operatorname{dim} \mathbb{A} \cap K=\operatorname{dim} K$. This is a contradiction. We conclude that $r^{-1}$ has to be an integer.

\subsection{Structure of the conjugating map and conclusion of proof}

Let us recall what we have shown so far: The IFS $\Phi$ is conjugated via an affine map $h(x)=c x+d$ to an IFS $\Psi=\left\{g_{i}(x)=r_{i} x+t_{i}\right\}$ that is in so-called Pisot form [6, Definition 2.2] for some integer Pisot number. This means that for every $g_{i} \in \Psi, g_{i}(x)=r^{n_{i}} x+t_{i}$ where $r^{-1}=n$ is an integer, the $\left(n_{i}\right)$ are relatively prime, and, since $r^{-1}$ is an integer, the translations $t_{i}$ have the form $t_{i}=\frac{z_{i}}{n^{s_{i}}}$ where $z_{i} \in \mathbb{Z}$ and $s_{i} \in \mathbb{N} \cup\{0\}$.

In this section we complete the proof of Theorem 1.3 by showing that we must have $c \in \mathbb{Q}$ and that $d$ is not an $n$-normal number. As we will see, this implies that every translation in the original IFS is not $n$-normal. Afterwards, we show that under some extra assumptions on $\Phi$ each such translation must be rational. So, let $X$ denote the attractor of $\Psi$. Since our IFS $\Phi$ is conjugated by the affine map $h(x)=c x+d$ to $\Psi$, we have

$$
h(X)=K=K_{\Phi} .
$$

Let $\epsilon>0$ and produce an IFS $\Lambda$ as in Lemma 7.5 but for the IFS $\Psi$. In particular,

$$
\operatorname{dim} X_{\Lambda} \geq \operatorname{dim} X-\epsilon
$$

and all the maps in $\Lambda$ have the same contraction $n^{-k}$ for some $k \in \mathbb{N}$. Furthermore, since all the maps in $\Lambda$ are compositions of maps in $\Psi$, the translations of the maps in $\Lambda$ retain the structure of those in $\Psi$. We also note that $h\left(X_{\Lambda}\right) \subseteq K$.

Consider the conjugated IFS $\Theta=\left\{h \circ l_{i} \circ h^{-1}\right\}_{l_{i} \in \Lambda}$ : Every affine map $f_{i} \in \Theta$ has the same contraction ratio $n^{-k}$, and its translation $f_{i}(0)=b_{i}$ satisfies, by the known structure of the maps in $\Lambda$,

$$
b_{i}=\frac{c \cdot z_{i}}{n^{s_{i}}}+d-\frac{d}{n^{k}}, \quad z_{i} \in \mathbb{Z}, s_{i} \in \mathbb{N} \cup\{0\} .
$$

Now, since $\Phi$ is non-trivial we may assume that so is $\Psi$, and consequently $\Lambda$ is non-trivial (if $\epsilon$ is small enough). So, there exist maps $f_{i}, f_{j} \in \Theta$ with $b_{i} \neq b_{j}$. This implies that

$$
0 \neq n^{k} \cdot b_{i}-n^{k} \cdot b_{j}=n^{k} \cdot c \cdot\left(\frac{z_{i}}{n^{s_{i}}}-\frac{z_{j}}{n^{s_{j}}}\right), \quad z_{i}, z_{j} \in \mathbb{Z}, s_{i}, s_{j} \in \mathbb{N} .
$$

By the last displayed equation, if $c \notin \mathbb{Q}$ then

$$
\left\{n^{k} b_{i}-n^{k} b_{j}: i, j \in \Phi\right\} \text { is not contained in a proper closed subgroup of } \mathbb{T} \text {. }
$$

Now, Let $\mu$ be a self similar measure on $h\left(X_{\Lambda}\right)$ with respect to the conjugated IFS $\Theta$, such that $\operatorname{dim} \mu=\operatorname{dim} X_{\Lambda}$. By [19, Theorem 4], which we may apply via (56), $\mu$ is pointwise $n$-normal. It is 
well known that this implies that $\mu$ is pointwise normal to all integer bases $p$ such that $p \sim n$ (see e.g. [55]). We also note that $\Theta$ satisfies the open set condition: Since $\Lambda$ has the strong separation condition, $X_{\Lambda}$ has positive Hausdorff measure in its dimension. Therefore, the attractor of $\Theta$, $h\left(X_{\Lambda}\right)$, also has positive Hausdorff measure in its dimension. Therefore, by [44, Theorem 1.1], $\Theta$ satisfies the open set condition. So, since all maps in the regular IFS $\Theta$ have contraction $\frac{1}{n^{k}}$, we may appeal to [29, Theorem 1.4] to see that $\mu$ is pointwise normal to all bases $p \geq 2$ such that $p \nsim n$. Therefore, $\mu(\mathbb{A})=1$, and we conclude that

$$
\operatorname{dim} K \cap \mathbb{A}=\operatorname{dim} h(X) \cap \mathbb{A} \geq \operatorname{dim} h\left(X_{\Lambda}\right) \cap \mathbb{A} \geq \operatorname{dim} \mu=\operatorname{dim} X_{\Lambda} \geq \operatorname{dim} X-\epsilon=\operatorname{dim} K-\epsilon .
$$

Taking $\epsilon \rightarrow 0$, we obtain a contradiction.

So far, we have established the structure of the conjugated IFS $\Psi$, and found that the conjugating map $h(x)=c x+d$ must have $c \in \mathbb{Q}$. We next show that $d$ cannot be $n$-normal. If $d$ is $n$-normal, then every translation $b_{i}$ in the homogeneous IFS $\Theta$ as above must be $n$-normal: Indeed, we have seen that

$$
b_{i}=\frac{c \cdot z_{i}}{n^{s_{i}}}+d\left(1-\frac{1}{n^{k}}\right), \quad z_{i} \in \mathbb{Z}, s_{i} \in \mathbb{N} \cup\{0\}
$$

and so $b_{i}=s \cdot d+t$ where $s, t \in \mathbb{Q}$ and $s \neq 0$. This implies that $b_{i}$ is $n$-normal as proved by Wall in his Ph.D. thesis [68]. We can now run the same argument as above, with the only difference being that since $b_{i}-b_{j} \in \mathbb{Q}$ and $b_{i}$ is $n$-normal for all $i, j$, we can use [19, Theorem 7] instead of [19, Theorem 4] to conclude that $\mu$ is pointwise absolutely normal. We have just shown that this leads to a contradiction. So, $d$ cannot be $n$-normal.

Finally, we have shown that $\Phi=\left\{h \circ g_{i} \circ h^{-1}\right\}_{g_{i} \in \Psi}$ where $\Psi=\left\{g_{i}(x)=r_{i} x+t_{i}\right\}$ is such that for every $g_{i} \in \Psi, g_{i}(x)=\frac{x}{n^{k_{i}}}+t_{i}$ where $\left(k_{i}\right)$ are relatively prime, and $t_{i}=\frac{z_{i}}{n^{s_{i}}}$ where $z_{i} \in \mathbb{Z}$ and $s_{i} \in \mathbb{N} \cup\{0\}$. We have also shown that $h$ is an affine map such that $h^{\prime}(0)=c \in \mathbb{Q}$ and $h(0)=d$ is not $n$-normal. So, every map $h \circ g_{i} \circ h^{-1}$ in $\Phi$ is of the form

$$
x \mapsto \frac{x}{n^{k_{i}}}+\frac{c \cdot z_{i}}{n^{s_{i}}}+d-\frac{d}{n^{k_{i}}} .
$$

Appealing to Wall's thesis [68] once more, since $d$ is not $n$ normal and $c \in \mathbb{Q}, \frac{c \cdot z_{i}}{n^{s_{i}}}+d-\frac{d}{n^{k_{i}}}$ is also not $n$-normal.

If furthermore there are two maps in $\Phi$ with different contraction ratios $\frac{1}{n^{k_{i}}} \neq \frac{1}{n^{k_{j}}}$ then their translations $t_{i}, t_{j}$ satisfy that, since $c \in \mathbb{Q}$

$$
n^{k_{i}} t_{i}-n^{k_{j}} t_{j}=q_{i, j}+\left(n^{k_{i}}-n^{k_{j}}\right) \cdot d, \text { where } q_{i, j} \in \mathbb{Q} .
$$

So, if $d \notin \mathbb{Q}$ then $n^{k_{i}} t_{i}-n^{k_{j}} t_{j} \notin \mathbb{Q}$ and thus

$$
\left\{n^{k_{i}} t_{i}-n^{k_{j}} t_{j}: i, j \in \Phi\right\} \text { is not contained in a proper closed subgroup of } \mathbb{T} \text {. }
$$

Now, assuming that $\Phi$ is regular, let $\mu$ be a self similar measure such that $\operatorname{dim} \mu=\operatorname{dim} K$. Then $\mu$ a.e. $x$ is $n$-normal by [19, Theorem 4] which applies via (57). Also, $\mu$ a.e. $x$ is $p$-normal for all $p \neq n$ via [29, Theorem 1.4], since $\Phi$ is regular and all maps in $\Phi$ have contractions that are independent of $p$. It follows that $\mu(\mathbb{A})=1$ so $\operatorname{dim} K \cap \mathbb{A}=\operatorname{dim} K$, a contradiction. We conclude that in this situation $d \in \mathbb{Q}$, and with this the proof of Theorem 1.3 is done. 


\subsection{Proof of Claim 7.4}

We now prove Claim 7.4. We work with the same notations introduced before Claim 7.4, Recall that for $\mathbf{p}$, a probability vector on the IFS $\Phi$ (which is a finite set), $\mu_{\mathbf{p}}$ is the corresponding self similar measure. By combining the results of Peres-Solomyak [46] and of Feng-Hu [24] (or by [28]), we see that the map $\mathbf{p} \mapsto \operatorname{dim} \mu_{\mathbf{p}}$ is lower semi-continuous. Notice that this holds true even if some of the entries in $\mathbf{p}$ are zero. Therefore, if $\mathbf{p}_{k} \rightarrow \mathbf{p}$ then

$$
\lim \inf \operatorname{dim} \mu_{\mathbf{p}_{k}} \geq \operatorname{dim} \mu_{\mathbf{p}}
$$

Now, let $\epsilon>0$. It follows from [57, Lemma 4.2] that there exists an IFS $\Lambda$ with strong separation such that every $f \in \Lambda$ is a composition of maps from $\Phi$, and $K_{\Lambda} \subseteq K$ with $\operatorname{dim} K_{\Lambda} \geq \operatorname{dim} K-\epsilon$. Notice that unlike Lemma 7.5, here we do not require all the maps in $\Lambda$ to have the same contraction ratio. It is a consequence of the proof of [57, Lemma 4.2] that we can choose $\Lambda$ by taking $m$ large enough and choosing a subset of the maps that make up the IFS $\Phi_{m}$. That is, $\Lambda \subseteq \Phi_{m}$ for some $m$.

Since $\Lambda$ has strong separation, we can always find a self similar measure $\mu_{\mathbf{p}}$ such that $\operatorname{dim} K_{\Lambda}=$ $\operatorname{dim} \mu_{\mathbf{p}}$. Since $\Lambda \subseteq \Phi_{m}$, we can find a sequence $\mathbf{p}_{k} \rightarrow \mathbf{p}$ such that each $\mathbf{p}_{k}$ is a strictly positive probability vector on $\Phi_{m}$. By the lower semi-continuity alluded to in the first paragraph, for every $k$ large enough we thus have

$$
\operatorname{dim} \mu_{\mathbf{p}_{k}} \geq \operatorname{dim} \mu_{\mathbf{p}}-\epsilon=\operatorname{dim} K_{\Lambda}-\epsilon \geq \operatorname{dim} K-2 \epsilon
$$

which implies Claim 7.4 .

\section{References}

[1] Jürgen Angst and Guillaume Poly. A weak Cramér condition and application to Edgeworth expansions. Electron. J. Probab., 22:Paper No. 59, 24, 2017.

[2] Artur Avila, Jairo Bochi, and Jean-Christophe Yoccoz. Uniformly hyperbolic finite-valued SL $(2, \mathbb{R})$-cocycles. Comment. Math. Helv., 85(4):813-884, 2010.

[3] Yves Benoist and Jean-François Quint. Random walks on reductive groups, volume 62 of Ergebnisse der Mathematik und ihrer Grenzgebiete. 3. Folge. A Series of Modern Surveys in Mathematics [Results in Mathematics and Related Areas. 3rd Series. A Series of Modern Surveys in Mathematics]. Springer, Cham, 2016.

[4] Christopher J. Bishop and Yuval Peres. Fractals in probability and analysis, volume 162. Cambridge University Press, 2016.

[5] Jean Bourgain and Semyon Dyatlov. Fourier dimension and spectral gaps for hyperbolic surfaces. Geom. Funct. Anal., 27(4):744-771, 2017.

[6] Julien Brémont. On the rajchman property for self-similar measures. arXiv preprint arXiv:1910.03463, 2019.

[7] E. Breuillard. Distributions diophantiennes et théorème limite local sur $\mathbb{R}^{d}$. Probab. Theory Related Fields, 132(1):39-73, 2005.

[8] Emmanuel Breuillard and Péter P. Varjú. On the dimension of Bernoulli convolutions. Ann. Probab., 47(4):2582-2617, 2019. 
[9] Ryan Broderick, Yann Bugeaud, Lior Fishman, Dmitry Kleinbock, and Barak Weiss. Schmidt's game, fractals, and numbers normal to no base. Math. Res. Lett., 17(2):307-321, 2010.

[10] Gavin Brown, William Moran, and Charles E. M. Pearce. Riesz products and normal numbers. J. London Math. Soc. (2), 32(1):12-18, 1985.

[11] Gavin Brown, William Moran, and Charles E. M. Pearce. Riesz products, Hausdorff dimension and normal numbers. Math. Proc. Cambridge Philos. Soc., 101(3):529-540, 1987.

[12] Alexander I. Bufetov and Boris Solomyak. On the modulus of continuity for spectral measures in substitution dynamics. Adv. Math., 260:84-129, 2014.

[13] Yann Bugeaud. Distribution modulo one and Diophantine approximation, volume 193 of Cambridge Tracts in Mathematics. Cambridge University Press, Cambridge, 2012.

[14] J. W. S. Cassels. On a problem of Steinhaus about normal numbers. Colloq. Math., 7:95-101, 1959.

[15] Yuanyang Chang and Xiang Gao. Fourier decay bound and differential images of self-similar measures. arXiv preprint arXiv:1710.07131, 2017.

[16] Xin-Rong Dai. When does a Bernoulli convolution admit a spectrum? Adv. Math., 231(34):1681-1693, 2012.

[17] Xin-Rong Dai, De-Jun Feng, and Yang Wang. Refinable functions with non-integer dilations. J. Funct. Anal., 250(1):1-20, 2007.

[18] H. Davenport, P. Erdős, and W. J. LeVeque. On Weyl's criterion for uniform distribution. Michigan Math. J., 10:311-314, 1963.

[19] Yiftach Dayan, Arijit Ganguly, and Barak Weiss. Random walks on tori and normal numbers in self similar sets. arXiv preprint arXiv:2002.00455, 2020.

[20] Dmitry Dolgopyat. Prevalence of rapid mixing in hyperbolic flows. Ergodic Theory Dynam. Systems, 18(5):1097-1114, 1998.

[21] Paul Erdös. On a family of symmetric Bernoulli convolutions. Amer. J. Math., 61:974-976, 1939.

[22] Paul Erdös. On the smoothness properties of a family of Bernoulli convolutions. Amer. J. Math., 62:180-186, 1940.

[23] Carl-Gustav Esseen. On the Liapounoff limit of error in the theory of probability. Ark. Mat. Astr. Fys., 28A(9):19, 1942.

[24] De-Jun Feng and Huyi Hu. Dimension theory of iterated function systems. Comm. Pure Appl. Math., 62(11):1435-1500, 2009.

[25] De-Jun Feng and Ka-Sing Lau. Multifractal formalism for self-similar measures with weak separation condition. J. Math. Pures Appl. (9), 92(4):407-428, 2009.

[26] Michael Hochman. On self-similar sets with overlaps and inverse theorems for entropy. Ann. of Math. (2), 180(2):773-822, 2014. 
[27] Michael Hochman. A short proof of Host's equidistribution theorem. arXiv preprint arXiv:2103.08938, 2021.

[28] Michael Hochman and Pablo Shmerkin. Local entropy averages and projections of fractal measures. Ann. of Math. (2), 175(3):1001-1059, 2012.

[29] Michael Hochman and Pablo Shmerkin. Equidistribution from fractal measures. Inventiones mathematicae, 202(1):427-479, 2015.

[30] Bernard Host. Nombres normaux, entropie, translations. Israel J. Math., 91(1-3):419-428, 1995.

[31] Thomas Jordan and Tuomas Sahlsten. Fourier transforms of Gibbs measures for the Gauss map. Math. Ann., 364(3-4):983-1023, 2016.

[32] J.-P. Kahane. Sur la distribution de certaines séries aléatoires. In Colloque de Théorie des Nombres (Univ. Bordeaux, Bordeaux, 1969), pages 119-122. 1971.

[33] R. Kaufman. Continued fractions and Fourier transforms. Mathematika, 27(2):262-267 (1981), 1980 .

[34] Robert Kaufman. On Bernoulli convolutions. In Conference in modern analysis and probability (New Haven, Conn., 1982), volume 26 of Contemp. Math., pages 217-222. Amer. Math. Soc., Providence, RI, 1984.

[35] Harry Kesten. Renewal theory for functionals of a Markov chain with general state space. Ann. Probability, 2:355-386, 1974.

[36] Jialun Li. Decrease of Fourier coefficients of stationary measures. Math. Ann., 372(3-4):1189$1238,2018$.

[37] Jialun Li. Fourier decay, renewal theorem and spectral gaps for random walks on split semisimple lie groups. To appear in Ann. Sci. Éc. Norm. Supér., 2018.

[38] Jialun Li, Frederic Naud, and Wenyu Pan. Kleinian schottky groups, patterson-sullivan measures and fourier decay. To appear in Duke math. J.

[39] Jialun Li and Tuomas Sahlsten. Fourier transform of self-affine measures. Adv. Math., $374: 107349,35,2020$.

[40] Jialun Li and Tuomas Sahlsten. Trigonometric series and self-similar sets. To appear in Journal of the European Mathematical Society, 2021.

[41] Russell Lyons. Seventy years of Rajchman measures. In Proceedings of the Conference in Honor of Jean-Pierre Kahane (Orsay, 1993), number Special Issue, pages 363-377, 1995.

[42] Jürgen Moser. On commuting circle mappings and simultaneous Diophantine approximations. Math. Z., 205(1):105-121, 1990.

[43] Carolina A. Mosquera and Pablo S. Shmerkin. Self-similar measures: asymptotic bounds for the dimension and Fourier decay of smooth images. Ann. Acad. Sci. Fenn. Math., 43(2):823$834,2018$. 
[44] Yuval Peres, MichałRams, Károly Simon, and Boris Solomyak. Equivalence of positive Hausdorff measure and the open set condition for self-conformal sets. Proc. Amer. Math. Soc., 129(9):2689-2699, 2001.

[45] Yuval Peres and Pablo Shmerkin. Resonance between Cantor sets. Ergodic Theory Dynam. Systems, 29(1):201-221, 2009.

[46] Yuval Peres and Boris Solomyak. Existence of $L^{q}$ dimensions and entropy dimension for selfconformal measures. Indiana Univ. Math. J., 49(4):1603-1621, 2000.

[47] A. D. Pollington. The Hausdorff dimension of a set of normal numbers. II. J. Austral. Math. Soc. Ser. A, 44(2):259-264, 1988.

[48] Martine Queffélec and Olivier Ramaré. Analyse de Fourier des fractions continues à quotients restreints. Enseign. Math. (2), 49(3-4):335-356, 2003.

[49] Ariel Rapaport. Proof of the exact overlaps conjecture for systems with algebraic contractions. to appear in Annales Scientifique de l'ENS.

[50] Ariel Rapaport and Péter P Varjú. Self-similar measures associated to a homogeneous system of three maps. arXiv preprint arXiv:2010.01022, 2020.

[51] Tuomas Sahlsten and Connor Stevens. Fourier decay in nonlinear dynamics. arXiv preprint arXiv:1810.01378, 2018.

[52] Tuomas Sahlsten and Connor Stevens. Fourier transform and expanding maps on cantor sets. arXiv preprint arXiv:2009.01703, 2020.

[53] R. Salem. Sets of uniqueness and sets of multiplicity. Trans. Amer. Math. Soc., 54:218-228, 1943.

[54] Raphaël Salem and Antoni Zygmund. Sur un théorème de Piatetçki-Shapiro. C. R. Acad. Sci. Paris, 240:2040-2042, 1955.

[55] Wolfgang M. Schmidt. On normal numbers. Pacific J. Math., 10:661-672, 1960.

[56] Wolfgang M. Schmidt. On badly approximable numbers and certain games. Trans. Amer. Math. Soc., 123:178-199, 1966.

[57] Pablo Shmerkin. Projections of Self-Similar and Related Fractals: A Survey of Recent Developments, pages 53-74. Springer International Publishing, Cham, 2015.

[58] Pablo Shmerkin. On Furstenberg's intersection conjecture, self-similar measures, and the $L^{q}$ norms of convolutions. Ann. of Math. (2), 189(2):319-391, 2019.

[59] Károly Simon. Overlapping cylinders: the size of a dynamically defined Cantor-set. In Ergodic theory of $\mathbf{Z}^{d}$ actions (Warwick, 1993-1994), volume 228 of London Math. Soc. Lecture Note Ser., pages 259-272. Cambridge Univ. Press, Cambridge, 1996.

[60] Boris Solomyak. Fourier decay for self-similar measures. Proc. Amer. Math. Soc., 149(8):32773291, 2021.

[61] Robert S. Strichartz. Self-similar measures and their Fourier transforms. I. Indiana Univ. Math. J., 39(3):797-817, 1990. 
[62] Robert S. Strichartz. Self-similar measures and their Fourier transforms. II. Trans. Amer. Math. Soc., 336(1):335-361, 1993.

[63] Robert S. Strichartz. Self-similar measures and their Fourier transforms. III. Indiana Univ. Math. J., 42(2):367-411, 1993.

[64] Masato Tsujii. On the Fourier transforms of self-similar measures. Dyn. Syst., 30(4):468-484, 2015 .

[65] Péter P. Varjú. On the dimension of Bernoulli convolutions for all transcendental parameters. Ann. of Math. (2), 189(3):1001-1011, 2019.

[66] Péter P Varjú and Han Yu. Fourier decay of self-similar measures and self-similar sets of uniqueness. To appear in Analysis and PDE.

[67] I. I. Šapiro Pyateckiı̌. On uniqueness of expansion of a function in trigonometric series. Doklady Akad. Nauk SSSR (N.S.), 85:497-500, 1952.

[68] Donald D. Wall. Normal Numbers. ProQuest LLC, Ann Arbor, MI, 1950. Thesis (Ph.D.)University of California, Berkeley.

[69] Jean-Christophe Yoccoz. Some questions and remarks about $\operatorname{SL}(2, \mathbb{R})$ cocycles. In Modern dynamical systems and applications, pages 447-458. Cambridge Univ. Press, Cambridge, 2004.

Department of Mathematics, the Pennsylvania State University, University Park, PA 16802, USA

E-mail address aka5983@psu.edu

E-mail address hertz@math.psu.edu

E-mail address zhirenw@psu.edu 